\title{
Energy Levels, Oscillator Strengths, and Transition Probabilities of Ni XIX and Cu XX
}

\author{
Wessameldin S. Abdelaziz ${ }^{*}$, M. Atta Khedr'1, Mai E. Ahmed2, Laila Gaabour ${ }^{3}$, \\ Tharwat El-Shirbini ${ }^{4}$ \\ ${ }^{1}$ National Institute of Laser Enhanced Sciences, Cairo University, Giza, Egypt \\ ${ }^{2}$ Environmental Affairs Agency, Cairo, Egypt \\ ${ }^{3}$ Physics Department, Faculty of science, King Abdul Aziz University, Jeddah, Saudi Arabia \\ ${ }^{4}$ Laboratory of Lasers and New Materials, Cairo University, Giza, Egypt \\ Email: *wessamlaser@yahoo.com
}

Received 28 December2013; revised 25 January 2014; accepted 21 February 2014

Copyright (C) 2014 by authors and Scientific Research Publishing Inc.

This work is licensed under the Creative Commons Attribution International License (CC BY). http://creativecommons.org/licenses/by/4.0/

(c) (i) Open Access

\section{Abstract}

Energy levels, oscillator strengths, and transition probabilities, for the $1 s^{2} 2 s^{2} 2 p^{6}, 2 p^{5} 31(1=0,1$, $2), 2 p^{54 l}(1=0,1,2,3)$ states in Ni XIX and $\mathrm{Cu} X X$ are calculated using COWAN code. The Correlation and relativistic effects are considered. The calculations are compared with other results in the literature. A good agreement is found. We also report on some unpublished energy values.

\section{Keywords}

Energy Levels; Oscillator Strengths; Transition Probabilities

\section{Introduction}

Almost coincident with the first observations of laser action in the IR and visible spectral regions in the 1960s, the search started for lasers operating at much shorter wavelengths. Measurements of definitive high output lasing at wavelength shorter than the ultra-violet were elusive, until the mid 1980s when conclusive evidence for "X-ray laser" operating at $209 \AA$ was produced from neon-like selenium [1].

In recent years, due to their peculiar structure of closed shells, Ne-like ions have been widely applied in the laboratory and in astronomical plasmas. The laboratory application is shown by the successful X-ray laser in the energy level of $2 p^{5} 3 p-2 p^{5} 3 s$ of Ne-like ions based on the mechanism of collisional excitation of electros [2]. Since the 1990s, much progress in experimental techniques has been achieved, but experimental data of atomic parameters are still limited, and theoretical calculations are needed.

Corresponding author. 
Laser produced plasmas are now well-known as suitable lasant media for amplification of soft X-ray energy range of electromagnetic spectrum. There are several schemes proposed and examined for producing laser plasma condition for X-ray lasing at shorter wavelengths with increasing efficiency. Plasma based recombination lasers [3] collisionally pumped [2] [3] are examples of such schemes. The dynamics of laser-produced plasma parameters such as the electron and ion temperatures and the density can be modeled by fluid hydrodynamic codes. Some examples of hydrodynamic codes include MEDUSA [4], and LASNEX [5]. Plasma transient collisionally pumped, using picosecond Chirped pulse amplification (CPA), X-ray lasers [6], using a capillary discharge [7], a free electron laser [8], optical field ionization of a gas cell [9] are also examples of such schemes. Among various pumping techniques for the $\mathrm{X}$-ray lasers, the collisional pumping of different materials in the Ne-like ionization state between the 3p-3s energy levels has shown a more stable and higher output.

The purpose of this work is to present the results of our calculations of energy levels, oscillator strengths, and transition probabilities of Ni XIX and Cu XX ions, and to compare the results with other in literature data.

\section{Computation of Atomic Structures}

\subsection{Model of Central Force Field}

In quantum mechanics, various physical processes can be summed by Schrödinger equation, i.e.

$$
H \Psi_{i}=E_{i} \Psi_{i} .
$$

In the non-relativistic case (the influence of relativistic effect will be discussed later), the Hamiltonian of an atomic system with $N$ electrons is:

$$
H=H_{\text {kin }}+H_{e-\text { nuc }}+H_{e-e}=\sum_{i} \frac{\hbar^{2}}{2 m_{e}} \nabla_{i}^{2}-\sum_{i} \frac{Z e^{2}}{r_{i}}+\sum_{i>j} \frac{e^{2}}{r_{i j}} .
$$

Here $H_{k i n}, H_{e-n u c}$ and $H_{e-e}$ refer, respectively, to the kinetic energy of electrons, the Coulomb potential and the energy of electrostatic interaction of electrons, $r_{i}$ is the distance between the $i$-th electron and nucleus, and $r_{i j}=\left|r_{i}-r_{j}\right|$.

By substituting the Hamiltonian into Schrödinger equation and solving the equation in the case of multiple electrons and multiple energy levels, the wave function is obtained. Now, due to the appearance of the term of interaction of electrons, an exact solution cannot be obtained. On the other hand, the interaction term is comparable with the Coulomb potential term, so it can by no means be ignored. An approximate solution is to adopt the method of central force field. If it is assumed that every electron moves in the central force field of the nucleus and also in the mean force field produced by other electrons, then we have the following effective Hamiltonian:

$$
H^{e f f}=\sum_{i}^{N} H_{i}^{e f f}=-\sum_{i=1}^{N}\left[\frac{1}{2} \frac{p_{i}^{2}}{m_{e}}+\frac{Z e^{2}}{r_{i}}-V_{i}^{e f f}\left(r_{i}\right)\right] .
$$

\subsection{Method of Calculation}

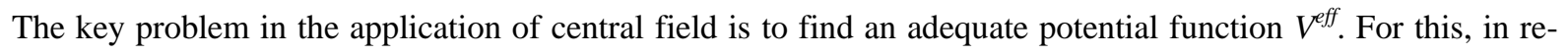
cent decades many effective method of calculation have been developed. Among them the more important ones are the potential model, Hartree-Fock theory, and the semi-empirical methods. In the following we present a brief introduction of semi-empirical methods.

Semi-empirical methods try to calculate atomic structures via solving the simplified form of the Hartree-Fock equation. The most typical is the Hartree-Fock-Slater method. Afterwards, Cowan et al. revised this method and developed the RCN/RCG program used in our work [10]. The merit of the program is its extreme effectiveness, and the shortcoming is its inability to estimate the precision.

\subsection{Configuration Interaction}

In the above-stated model of central force field, every electron can be described with a simple wave function. The overall wave function of atoms may be expressed with the following Slater determinant: 


$$
\Phi=\frac{1}{\sqrt{N !}}\left(\begin{array}{ccc}
\varphi_{1}\left(\chi_{1}\right) & \ldots & \varphi_{1}\left(\chi_{N}\right) \\
\vdots & \ddots & \vdots \\
\varphi_{N}\left(\chi_{1}\right) & \cdots & \varphi_{N}\left(\chi_{N}\right)
\end{array}\right) .
$$

In reality, such a description is not very precise. The best wave function should be a linear combination of wave functions with single configurations, and these wave functions possess the same total angular momentum and spin symmetry. This method is called the interaction of configurations. In the computation of atomic structures, consideration of the configuration interaction is the basis requirement for a program.

\subsection{Relativistic Correction}

In a non-relativistic system, the oscillator strengths and dipole transitions under LS-coupling can be calculated. In calculating forbidden transitions, jj-coupling must be used, and for this relativistic effects have to be taken into account. Generally speaking, the effects may be treated in two ways. One is inclusion of Breit-Pauli operator in the non-relativistic equation, and other is direct solution of the Dirac equation. For the former, a mass velocity term, the Darwin term caused by the electric moments of electrons and the spin-orbit term are added to the Hamiltonian of the model of central force field [11]. For relativistic correction, the program RCN/RCG restore to the Breit-Pauli correction.

\subsection{Weighted Oscillator Strengths and Lifetimes}

The oscillator strength $f\left(\gamma \gamma^{\prime}\right)$ is a physical quantity related to line intensity $I$ and transition probability $W\left(\gamma \gamma^{\prime}\right)$, by

$$
W\left(\gamma \gamma^{\prime}\right)=\frac{2 w^{2} e^{2}}{m c^{3}}\left|f\left(\gamma \gamma^{\prime}\right)\right|
$$

with, $\quad$ I $\alpha g W\left(\gamma \gamma^{\prime}\right) \alpha g\left|f\left(\gamma \gamma^{\prime}\right)\right|=g f$ Sobelman [12].

Here $m$ is electron mass, $e$ is electron charge, $\gamma$ is initial quantum state, $w=\left(E(\gamma)-E\left(\gamma^{\prime}\right)\right) / \hbar, E(\gamma)$ initial state energy, $g=(2 J+1)$ is the number of degenerate quantum state with angular momentum $J$ (in the formula for initial state).

Quantities with primes refer to the final state.

In the above equation, the weighted oscillator strength, $g f$, is given by Cowan [10]:

$$
g f=\frac{8 \pi^{2} m c a_{0}^{2} \sigma}{3 h} S,
$$

where $g$ is the statistical weight of lower level, $f$ is the absorption oscillator strength, $\sigma=\left(E(\gamma)-E\left(\gamma^{\prime}\right)\right) / h c, h$ is planck's constant, $c$ is light velocity, and $a_{0}$ is Bohr radius, and the electric dipole line strength is defined by:

$$
s=\left|\left\langle\lambda J\left\|P^{1}\right\| \gamma^{\prime} J^{\prime}\right\rangle\right|^{2} \text {. }
$$

This quantity is a measure of the total strength of the spectral line, including all possible transitions between $\mathrm{m}, \mathrm{m}$, for different $J_{z}$ Eigen states. The tensor operator $P^{1}$ (first order) in the reduced matrix element is the classical dipole moment for the atom in units of $e a_{0}$.

To obtain $g f$, we need to calculate $S$ first, (or its square root):

$$
s_{\gamma^{\prime}}^{1 / 2}=\left\langle\lambda J\left\|P^{1}\right\| \gamma^{\prime} J^{\prime}\right\rangle \text {. }
$$

In a multiconfiguration calculation we have to expand the wave function $|\gamma J\rangle$ for both upper and lower levels $|\beta J\rangle$.

In terms of single configuration wave functions, lower levels:

$$
|\gamma J\rangle=\sum_{\beta} y_{\beta J}^{\gamma}|\beta J\rangle
$$

Therefore, we can have the multiconfigurational expression for the square root of line strength: 


$$
s_{\gamma \gamma}^{1 / 2}=\sum_{\beta} \sum_{\beta} y_{\beta J}^{\gamma}\left\langle\beta J\left\|P^{1}\right\| \beta^{\backslash} J^{\backslash}\right\rangle .
$$

The probability per unit time of an atom in specific state $\gamma J$ to make a spontaneous transition to any state with lower energy is

$$
P(\gamma J)=\sum A\left(\gamma J, \gamma^{\backslash} J^{\backslash}\right)
$$

where $A\left(\gamma J, \gamma^{\backslash} J^{\backslash}\right)$ is the Einstein spontaneous emission transition state. $\gamma^{\backslash} J^{\backslash}$ to $\gamma J$ probability rate, for a transition from the

The sum is over all states $\gamma^{\prime} J^{\backslash}$ with $E\left(\gamma^{\backslash} J^{\backslash}\right)<E(\gamma J)$.

The Einstein probability rate is related to $g f$ with the following relation [13]:

$$
g A=\frac{8 \pi^{2} e^{2} \sigma^{2}}{m c} g f .
$$

Since the natural lifetime $\tau(\gamma J)$ is the inverse of transition probability, then:

$$
\tau(\gamma J)=\left(\sum A\left(\gamma J, \gamma^{\backslash} J^{\backslash}\right)\right)^{-1}
$$

This is applicable to an isolated atom.

Interaction with matter or radiation will reduce the lifetime of any state.

\section{Results and Discussions}

Adopting the program RCN/RCG [10], we have computed the parameters of atomic structures of Ni XIX and $\mathrm{Cu}$ XX respectively. The energy levels considered in the calculation have 65 fine structures ranging from ground state $1 s^{2} 2 s^{2} 2 p^{6}$ to $2 p^{5} 3 l(l=0,1,2)$ and $2 p^{5} 4 l(l=0,1,2,3)$ states. Our computation has yielded the energy level intervals of electric dipolar spectral transitions, oscillator strengths and transition probabilities. In our calculations of wave functions, the relativistic correction is taken into consideration. Our results are presented in Tables 1-6. Some new and previously unpublished energy levels are given in energy level tables.

Table 1. The Hartree-Fock parameters and fitted parameters for energy levels of $\mathrm{Ni}$ XIX.

\begin{tabular}{cccc}
\hline parameter & HF & LSF & LSF/HF \\
\hline $\mathrm{E}_{\mathrm{av}} 2 \mathrm{p}^{6}$ & 2.142 & 2.1383 & 0.998273 \\
$\mathrm{E}_{\mathrm{av}} 2 \mathrm{p}^{5} 3 \mathrm{p}$ & 7485.004 & 7482.363 & 0.999647 \\
$\zeta_{(2 \mathrm{p})}$ & 95.988 & 94.7867 & 0.987485 \\
$\zeta_{(\mathrm{pp})}$ & 21.438 & 22.2906 & 1.039771 \\
$\mathrm{~F}^{2}(2 \mathrm{p} 3 \mathrm{p})$ & 94.276 & 125.4261 & 1.330414 \\
$\mathrm{G}^{0}(2 \mathrm{p} 3 \mathrm{p})$ & 37.202 & 42.9558 & 1.154664 \\
$\mathrm{G}^{2}(2 \mathrm{p} 3 \mathrm{p})$ & 40.74 & 48.8414 & 1.198856 \\
$\mathrm{E}_{\mathrm{av}} 2 \mathrm{p}^{5} 4 \mathrm{p}$ & 9768.31 & 9768.302 & 0.999999 \\
$\zeta_{(2 \mathrm{p})}$ & 96.282 & 96.2777 & 0.999955 \\
$\zeta_{(4 \mathrm{p})}$ & 8.556 & 8.5566 & 1.00007 \\
$\mathrm{~F}^{2}(2 \mathrm{p} 4 \mathrm{p})$ & 34.073 & 34.0829 & 1.000291 \\
$\mathrm{G}^{0}(2 \mathrm{p} 4 \mathrm{p})$ & 11.939 & 11.933 & 0.999497 \\
$\mathrm{G}^{2}(2 \mathrm{p} 4 \mathrm{p})$ & 14.164 & 14.1784 & 1.001017 \\
$\mathrm{E}_{\mathrm{av}} 2 \mathrm{p}^{5} 4 \mathrm{f}$ & 9988.381 & 9988.391 & 1.000001 \\
$\zeta_{(2 \mathrm{p})}$ & 96.451 & 96.449 & 0.999979 \\
$\zeta_{(4 \mathrm{f})}$ & 0.289 & 0.2877 & 0.995502 \\
$\mathrm{~F}^{2}(2 \mathrm{p} 4 \mathrm{f})$ & 23.94 & 23.8338 & 0.995564 \\
$\mathrm{G}^{2}(2 \mathrm{p} 4 \mathrm{f})$ & 4.747 & 4.6954 & 0.98913 \\
$\mathrm{G}^{4}(2 \mathrm{p} 4 \mathrm{f})$ & 3.079 & 3.0733 & 0.998149 \\
$\mathrm{E}_{\mathrm{av}} 2 \mathrm{p}^{5} 3 \mathrm{~s}$ & 7161.305 & 7159.783 & 0.999787 \\
$\zeta_{(2 \mathrm{p})}$ & 95.984 & 94.8994 & 0.9887 \\
$\mathrm{G}^{1}(2 \mathrm{p} 3 \mathrm{~s})$ & 36.478 & 41.7018 & 1.143204 \\
\hline
\end{tabular}




\section{Continued}

\begin{tabular}{cccc}
\hline $\mathrm{E}_{\mathrm{av}} 2 \mathrm{p}^{5} 3 \mathrm{~d}$ & 7892.665 & 7889.081 & 0.999546 \\
$\zeta_{(2 \mathrm{p})}$ & 96.035 & 95.2727 & 0.992062 \\
$\zeta_{(3 \mathrm{~d})}$ & 2.508 & 2.3254 & 0.927193 \\
$\mathrm{~F}^{2}(2 \mathrm{p} 3 \mathrm{~d})$ & 120.269 & 142.4402 & 1.184347 \\
$\mathrm{G}^{1}(2 \mathrm{p} 3 \mathrm{~d})$ & 109.368 & 115.0901 & 1.05232 \\
$\mathrm{G}^{3}(2 \mathrm{p} 3 \mathrm{~d})$ & 63.043 & 71.8264 & 1.139324 \\
$\mathrm{E}_{\mathrm{av}} 2 \mathrm{p}^{5} 4 \mathrm{~s}$ & 9638.228 & 9634.162 & 0.999578 \\
$\zeta_{(2 \mathrm{p})}$ & 96.289 & 93.9094 & 0.975287 \\
$\mathrm{G}^{1}(2 \mathrm{p} 4 \mathrm{f})$ & 12.077 & 0 & 0 \\
$\mathrm{E}_{\mathrm{av}} 2 \mathrm{p}^{5} 4 \mathrm{~d}$ & 9920.106 & 9915.483 & 0.999534 \\
$\zeta_{(2 \mathrm{p})}$ & 96.306 & 93.9707 & 0.975751 \\
$\zeta_{(4 \mathrm{~d})}$ & 1.075 & 0.6049 & 0.562698 \\
$\mathrm{~F}^{2}(2 \mathrm{p} 4 \mathrm{~d})$ & 42.438 & 66.6911 & 1.571495 \\
$\mathrm{G}^{1}(2 \mathrm{p} 4 \mathrm{~d})$ & 39.057 & 32.991 & 0.844689 \\
$\mathrm{G}^{3}(2 \mathrm{p} 4 \mathrm{~d})$ & 23.303 & 11.5745 & 0.496696 \\
\hline
\end{tabular}

Table 2. The Hartree-Fock parameters and fitted parameters for energy levels of $\mathrm{Cu}$ $\mathrm{XX}$.

\begin{tabular}{|c|c|c|c|}
\hline parameter & $\mathrm{HF}$ & LSF & LSF/HF \\
\hline$E_{a v} 2 p^{6}$ & 2.138 & 2.1348 & 0.998503 \\
\hline$E_{a v} 2 p^{5} 3 p$ & 8186.271 & 8183.26 & 0.999632 \\
\hline$\zeta_{(2 \mathrm{p})}$ & 112.683 & 111.043 & 0.985446 \\
\hline$\zeta_{(3 \mathrm{p})}$ & 25.51 & 25.9186 & 1.016017 \\
\hline$F^{2}(2 p 3 p)$ & 99.216 & 138.302 & 1.393949 \\
\hline$G^{0}(2 p 3 p)$ & 39.1 & 45.3771 & 1.16054 \\
\hline$G^{2}(2 p 3 p)$ & 42.91 & 45.7448 & 1.066064 \\
\hline$E_{a v} 2 p^{5} 4 p$ & 10699.49 & 10699.48 & 0.999999 \\
\hline$\zeta_{(2 \mathrm{p})}$ & 113.019 & 113.013 & 0.999947 \\
\hline$\zeta_{(4 \mathrm{p})}$ & 10.204 & 10.2041 & 1.00001 \\
\hline$F^{2}(2 p 4 p)$ & 35.938 & 35.9537 & 1.000437 \\
\hline$G^{0}(2 p 4 p)$ & 12.541 & 12.5361 & 0.999609 \\
\hline$G^{2}(2 p 4 p)$ & 14.926 & 14.938 & 1.000804 \\
\hline$E_{a v} 2 p^{5} 4 f$ & 10933.57 & 10933.58 & 1.000001 \\
\hline$\zeta_{(2 \mathrm{p})}$ & 113.213 & 113.2098 & 0.999972 \\
\hline$\zeta_{(4 f)}$ & 0.354 & 0.3533 & 0.998023 \\
\hline$F^{2}(2 p 4 f)$ & 25.631 & 25.5072 & 0.99517 \\
\hline$G^{2}(2 p 4 f)$ & 5.185 & 5.1263 & 0.988679 \\
\hline$G^{4}(2 p 4 f)$ & 3.364 & 3.3573 & 0.998008 \\
\hline$E_{a v} 2 p^{5} 3 s$ & 7842.324 & 7840.28 & 0.999739 \\
\hline$\zeta_{(2 \mathrm{p})}$ & 112.68 & 111.1721 & 0.986618 \\
\hline $\mathrm{G}^{1}(2 \mathrm{p} 3 \mathrm{~s})$ & 38.31 & 44.2347 & 1.154652 \\
\hline $\mathrm{E}_{\mathrm{av}} 2 \mathrm{p}^{5} 3 \mathrm{~d}$ & 8616.991 & 8613.123 & 0.999551 \\
\hline$\zeta_{(2 p)}$ & 112.733 & 111.6444 & 0.990344 \\
\hline$\zeta_{(3 \mathrm{~d})}$ & 3.052 & 2.5801 & 0.84538 \\
\hline$F^{2}(2 p 3 d)$ & 127.306 & 160.6774 & 1.262135 \\
\hline $\mathrm{G}^{1}(2 \mathrm{p} 3 \mathrm{~d})$ & 116.36 & 126.6669 & 1.088578 \\
\hline$G^{3}(2 p 3 d)$ & 67.105 & 66.6409 & 0.993084 \\
\hline$E_{a v} 2 p^{5} 4 s$ & 10560.94 & 10558.6 & 0.999778 \\
\hline$\zeta_{(2 \mathrm{p})}$ & 113.028 & 110.7571 & 0.979909 \\
\hline$G^{1}(2 p 4 f)$ & 12.704 & 0 & 0 \\
\hline $\mathrm{E}_{\mathrm{av}} 2 \mathrm{p}^{5} 4 \mathrm{~d}$ & 10860.24 & 10859.29 & 0.999912 \\
\hline$\zeta_{(2 \mathrm{p})}$ & 113.046 & 113.2196 & 1.001536 \\
\hline$\zeta_{(4 \mathrm{~d})}$ & 1.307 & 1.7952 & 1.373527 \\
\hline$F^{2}(2 p 4 d)$ & 44.878 & 48.0615 & 1.070937 \\
\hline $\mathrm{G}^{1}(2 \mathrm{p} 4 \mathrm{~d})$ & 41.346 & 37.5198 & 0.907459 \\
\hline $\mathrm{G}^{3}(2 \mathrm{p} 4 \mathrm{~d})$ & 24.697 & 27.0868 & 1.096765 \\
\hline
\end{tabular}


फ

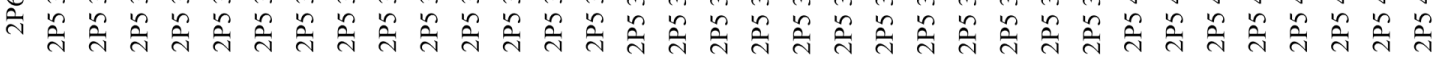

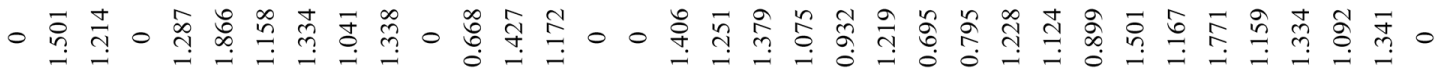

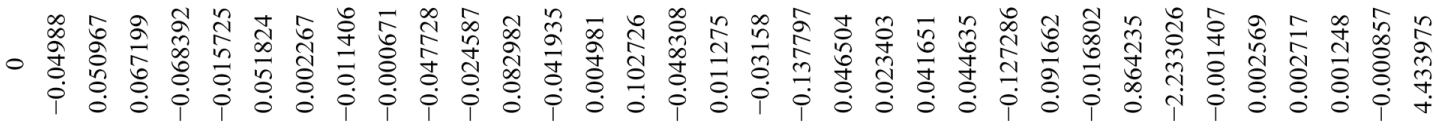

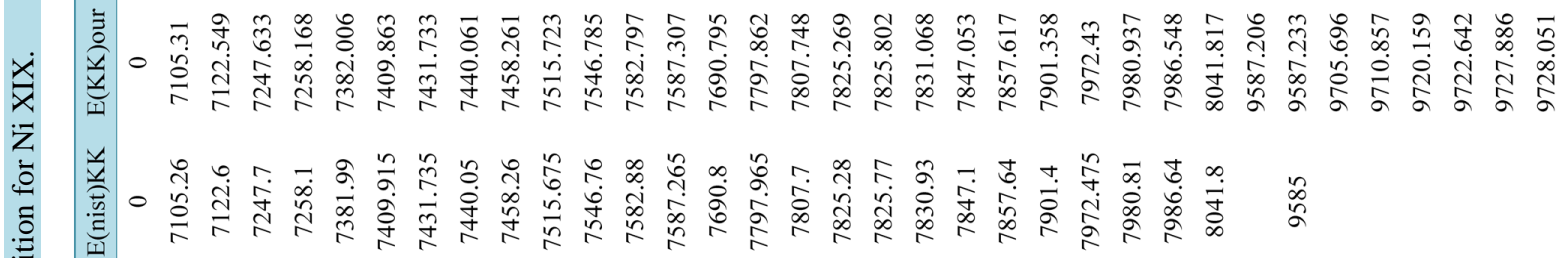

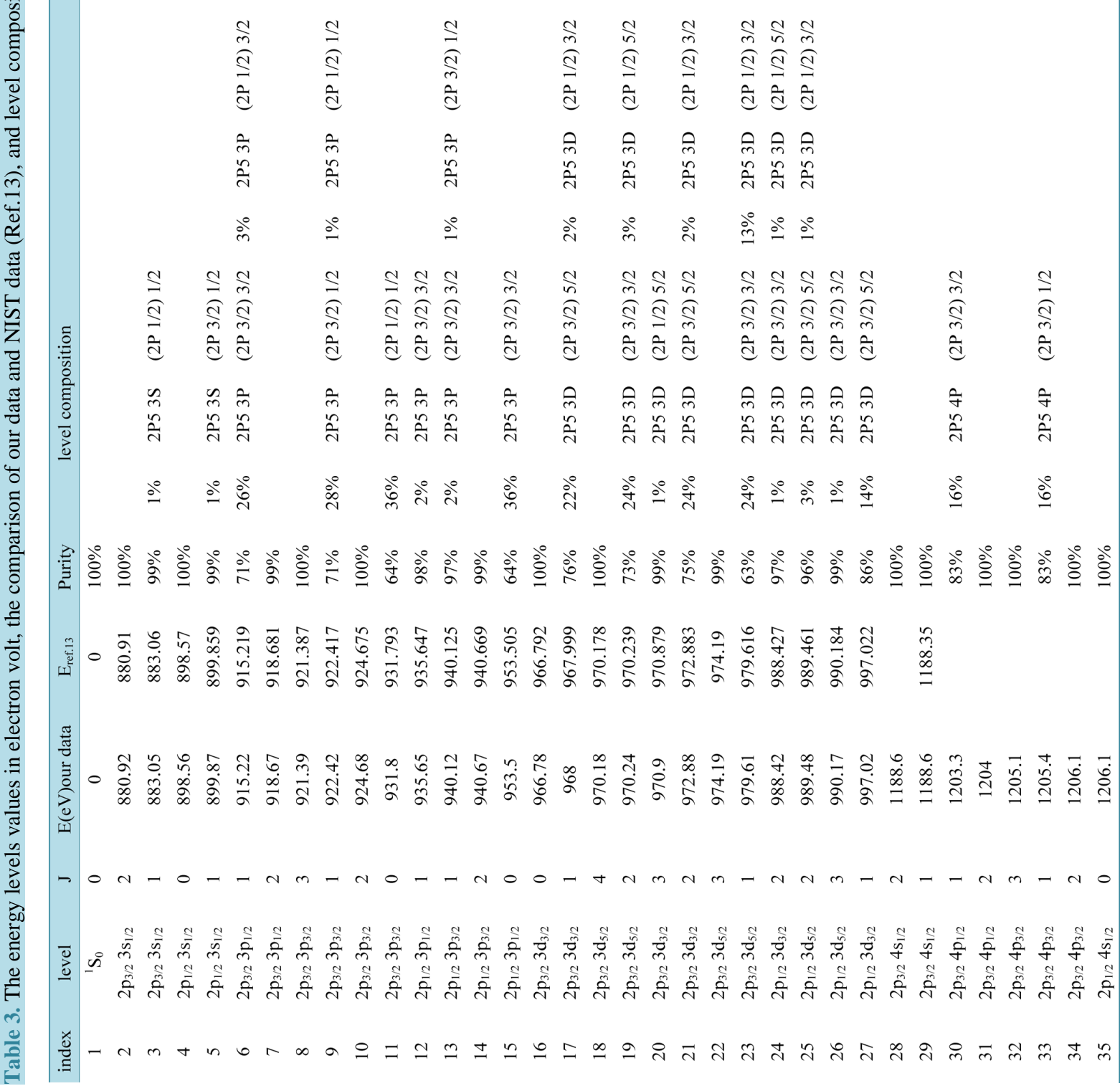




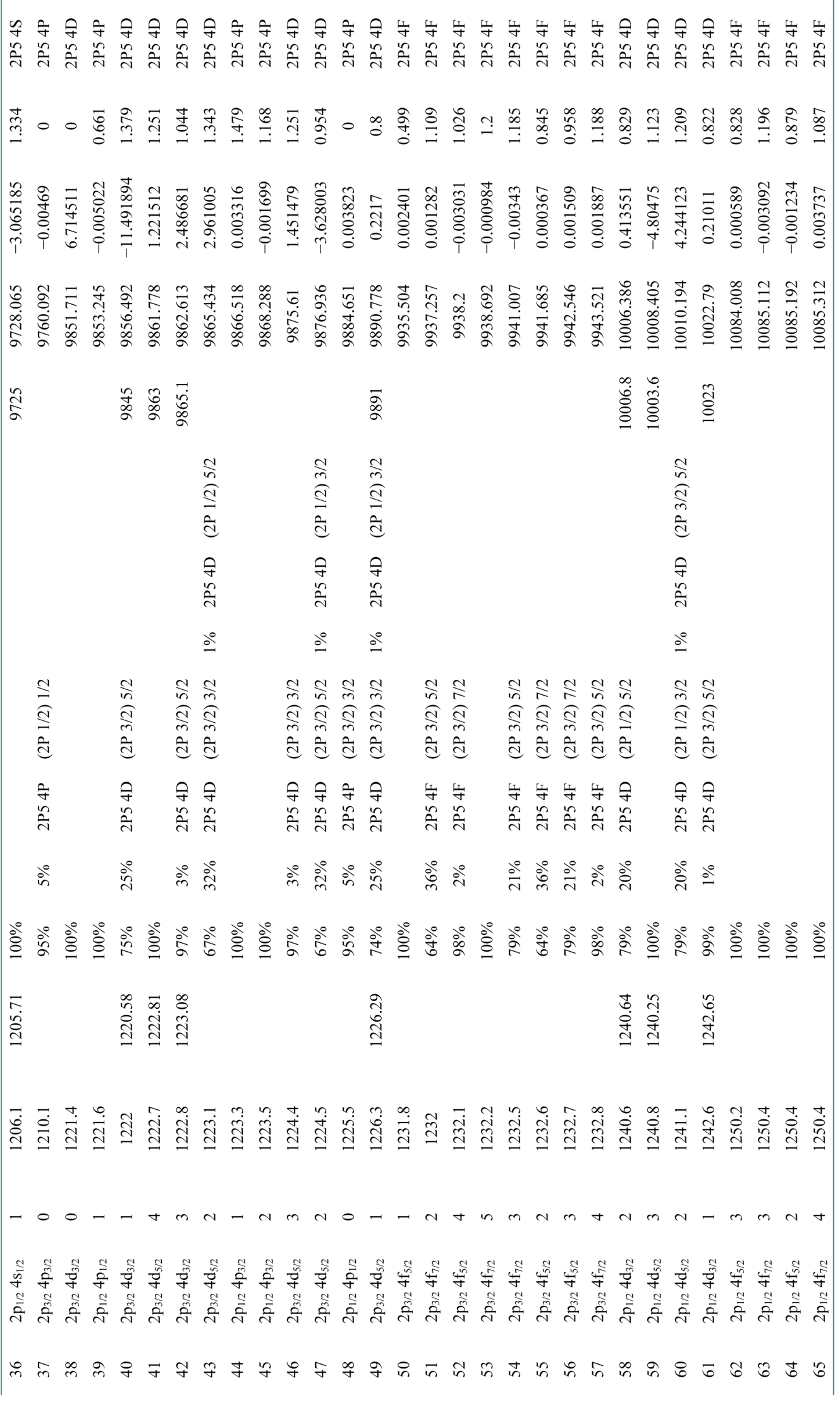




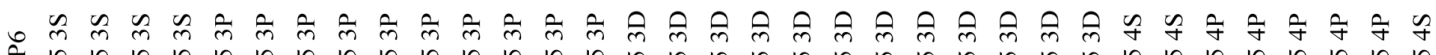

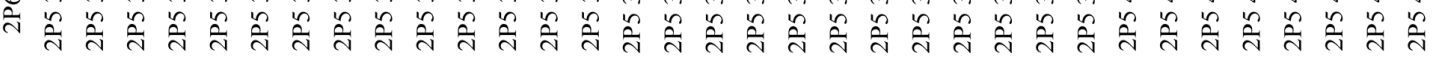
○ 官

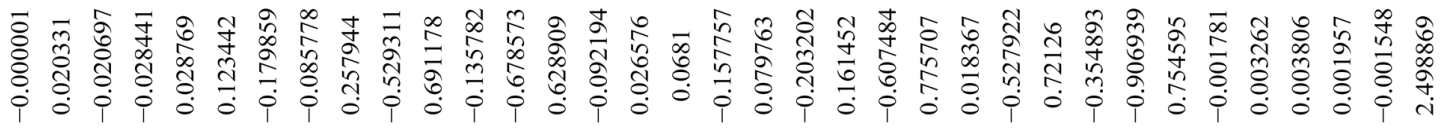

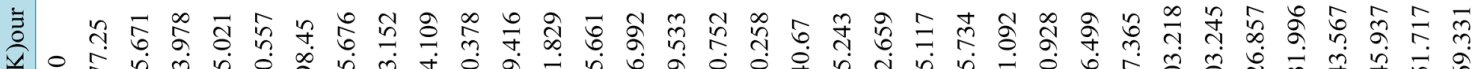

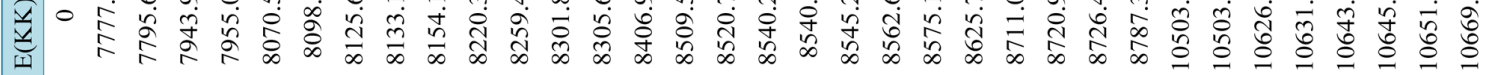

\begin{tabular}{|c|c|c|c|c|c|}
\hline 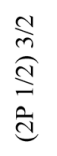 & $\begin{array}{l}\stackrel{\mathrm{d}}{\mathbb{d}} \\
\stackrel{\mathrm{d}}{\mathrm{d}}\end{array}$ & $\begin{array}{l}\stackrel{N}{J} \\
\frac{d}{d} \\
\hat{d}\end{array}$ & $\begin{array}{l}\frac{\mathrm{d}}{\mathrm{d}} \\
\frac{\mathrm{d}}{\mathrm{d}} \\
\stackrel{\mathrm{d}}{\mathrm{d}}\end{array}$ & $\begin{array}{l}\frac{1}{n} \\
\stackrel{\Omega}{S} \\
\stackrel{d}{d}\end{array}$ & 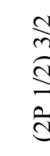 \\
\hline $\begin{array}{l}\hat{m} \\
\hat{n} \\
\hat{\sim}\end{array}$ & $\begin{array}{l}\hat{m} \\
\stackrel{n}{N}\end{array}$ & $\begin{array}{l}\hat{\bar{m}} \\
\stackrel{\tilde{n}}{1}\end{array}$ & 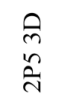 & $\begin{array}{l}\hat{i} \\
\hat{\sim}\end{array}$ & $\hat{\tilde{n}}$ \\
\hline$a^{\circ}$ & $\stackrel{\circ}{-}$ & $\stackrel{\circ}{\circ}$ & ふें & i & 0 \\
\hline
\end{tabular}

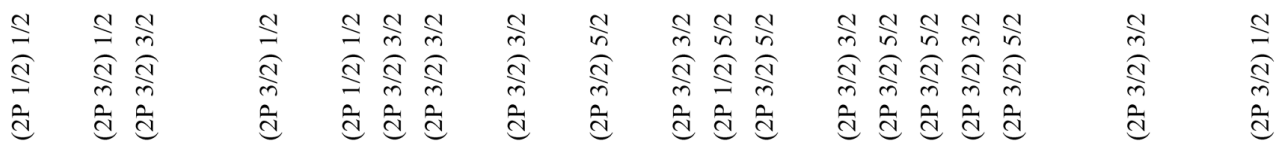

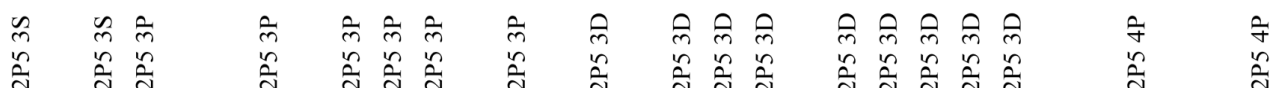

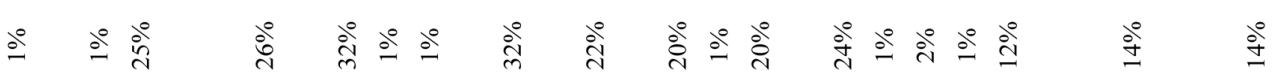

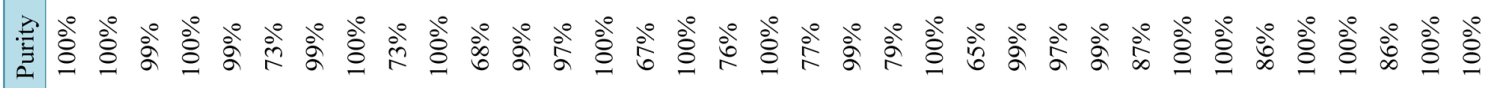

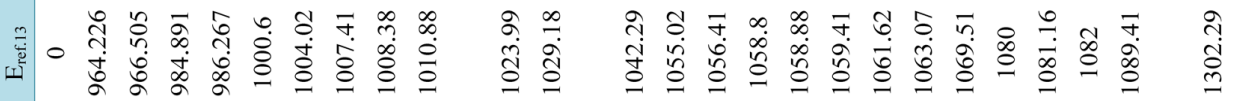

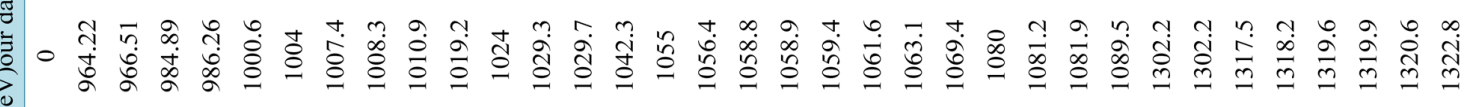

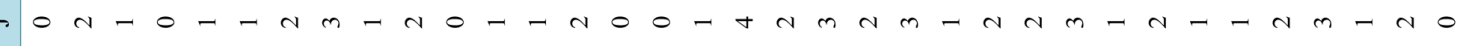

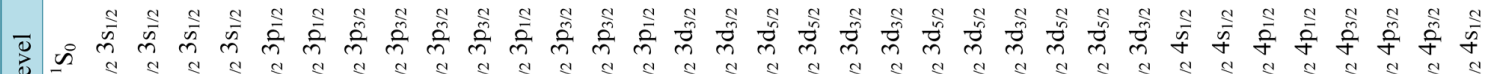

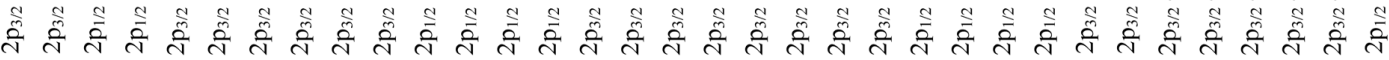

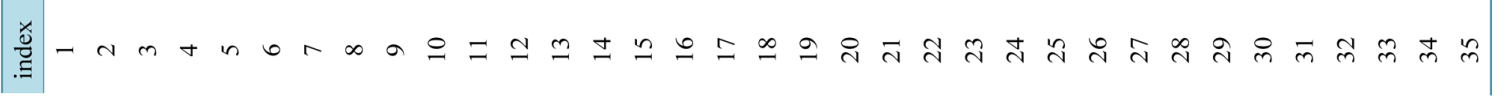




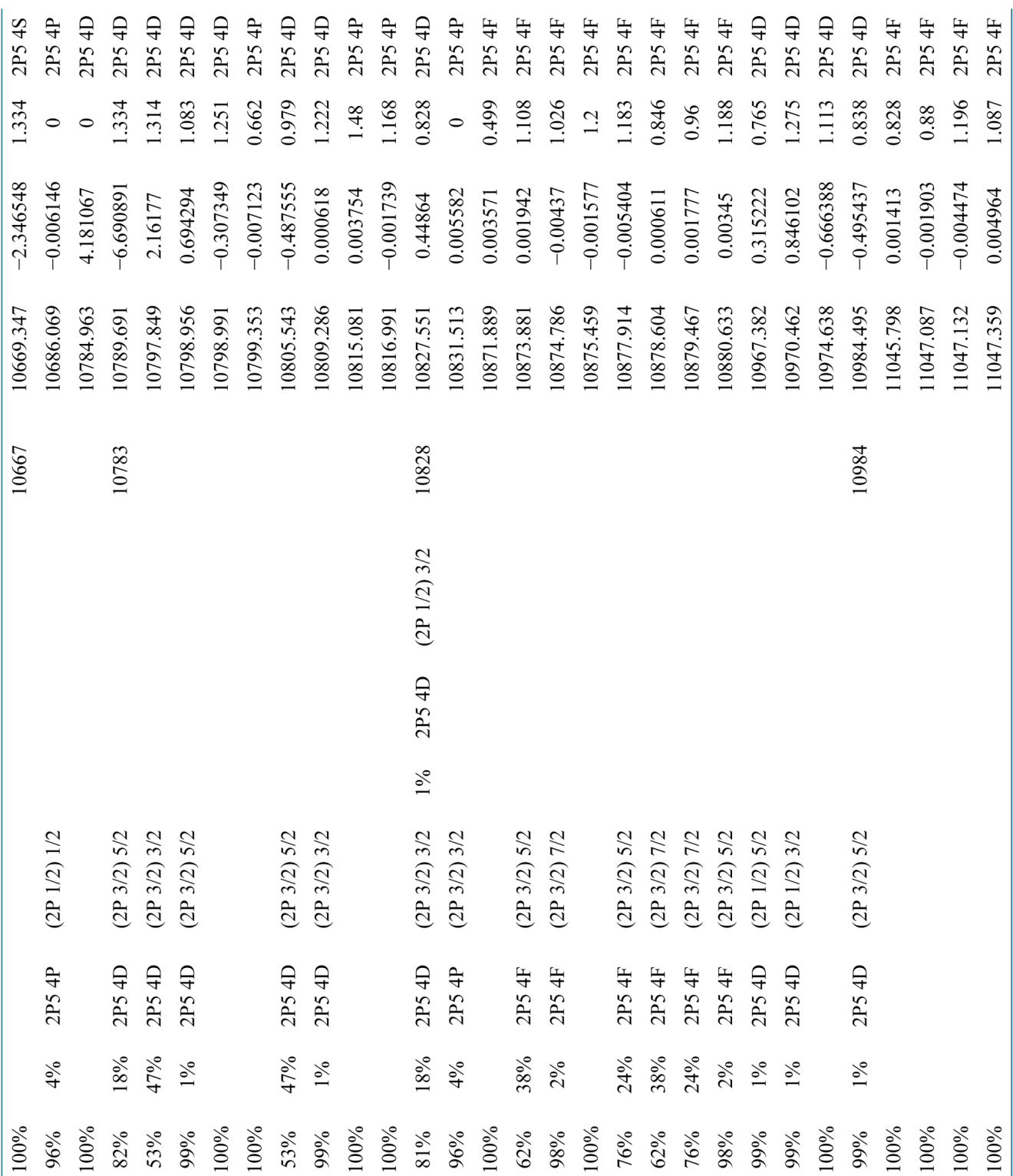

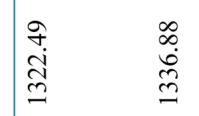

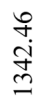

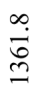

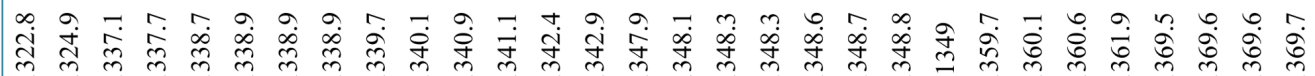

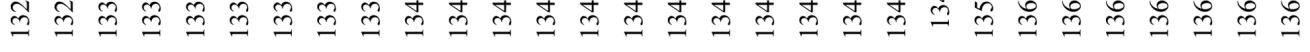

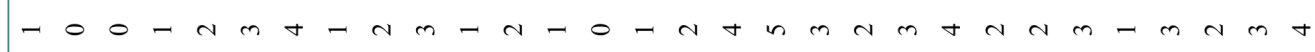

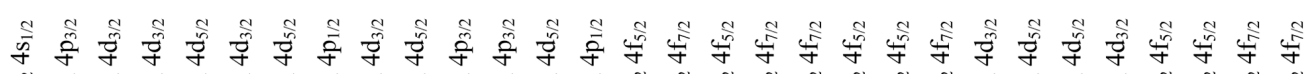

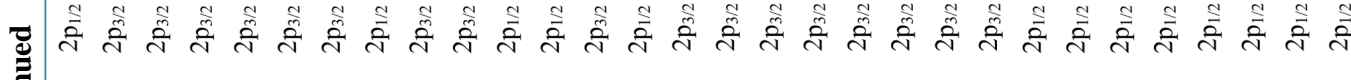

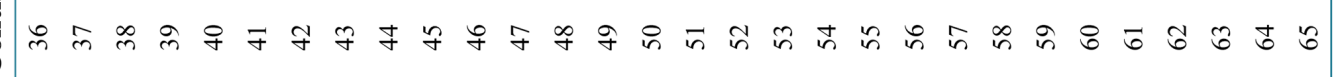


Table 5. The allowed electric dipole transitions for Ni XIX.

\begin{tabular}{|c|c|c|c|c|}
\hline i & $\mathrm{j}$ & $\lambda(\AA)$ & gf & Aji \\
\hline 1 & 3 & 14.04 & $1.02 \mathrm{E}-01$ & $1.2 \mathrm{E}+12$ \\
\hline 1 & 5 & 13.778 & $7.93 \mathrm{E}-02$ & $9.3 \mathrm{E}+11$ \\
\hline 1 & 17 & 12.808 & $1.29 \mathrm{E}-02$ & $1.7 \mathrm{E}+11$ \\
\hline 1 & 23 & 12.656 & $9.40 \mathrm{E}-01$ & $1.3 \mathrm{E}+13$ \\
\hline 1 & 27 & 12.435 & $2.32 \mathrm{E}+00$ & $3.3 \mathrm{E}+13$ \\
\hline 1 & 29 & 10.431 & $2.48 \mathrm{E}-02$ & $5.1 \mathrm{E}+11$ \\
\hline 1 & 36 & 10.28 & $1.34 \mathrm{E}-02$ & $2.8 \mathrm{E}+11$ \\
\hline 1 & 40 & 10.146 & $1.34 \mathrm{E}-02$ & $2.9 \mathrm{E}+11$ \\
\hline 1 & 49 & 10.11 & $5.01 \mathrm{E}-01$ & $1.1 \mathrm{E}+13$ \\
\hline 1 & 61 & 9.977 & $3.77 \mathrm{E}-01$ & $8.4 \mathrm{E}+12$ \\
\hline 2 & 6 & 361.41 & $2.76 \mathrm{E}-01$ & 4.7E+09 \\
\hline 2 & 7 & 328.35 & $2.45 \mathrm{E}-01$ & $3.0 \mathrm{E}+09$ \\
\hline 2 & 8 & 306.35 & $7.91 \mathrm{E}-01$ & $8.0 \mathrm{E}+09$ \\
\hline 2 & 9 & 298.73 & $6.12 \mathrm{E}-03$ & $1.5 \mathrm{E}+08$ \\
\hline 2 & 10 & 283.33 & $3.18 \mathrm{E}-01$ & $5.3 \mathrm{E}+09$ \\
\hline 2 & 12 & 226.51 & $3.85 \mathrm{E}-04$ & $1.7 \mathrm{E}+07$ \\
\hline 2 & 13 & 209.43 & $1.26 \mathrm{E}-02$ & $6.4 \mathrm{E}+08$ \\
\hline 2 & 14 & 207.47 & $2.71 \mathrm{E}-03$ & $8.4 \mathrm{E}+07$ \\
\hline 2 & 30 & 38.456 & $2.59 \mathrm{E}-01$ & $3.9 \mathrm{E}+11$ \\
\hline 2 & 31 & 38.38 & $1.98 \mathrm{E}-01$ & $1.8 \mathrm{E}+11$ \\
\hline 2 & 32 & 38.243 & $6.00 \mathrm{E}-01$ & $3.9 \mathrm{E}+11$ \\
\hline 2 & 33 & 38.207 & $5.61 \mathrm{E}-05$ & $8.5 \mathrm{E}+07$ \\
\hline 2 & 34 & 38.13 & $2.17 \mathrm{E}-01$ & $2.0 \mathrm{E}+11$ \\
\hline 2 & 39 & 36.391 & $1.56 \mathrm{E}-05$ & $2.6 \mathrm{E}+07$ \\
\hline 2 & 44 & 36.216 & $3.28 \mathrm{E}-04$ & $5.6 \mathrm{E}+08$ \\
\hline 2 & 45 & 36.193 & $6.43 \mathrm{E}-05$ & $6.5 \mathrm{E}+07$ \\
\hline 2 & 50 & 35.333 & $6.58 \mathrm{E}-06$ & $1.2 \mathrm{E}+07$ \\
\hline 2 & 51 & 35.311 & $7.59 \mathrm{E}-05$ & $8.1 \mathrm{E}+07$ \\
\hline 2 & 54 & 35.265 & $2.51 \mathrm{E}-04$ & $1.9 \mathrm{E}+08$ \\
\hline 2 & 55 & 35.256 & $2.02 \mathrm{E}-06$ & $2.2 \mathrm{E}+06$ \\
\hline 2 & 56 & 35.246 & $8.20 \mathrm{E}-06$ & $6.3 \mathrm{E}+06$ \\
\hline 2 & 62 & 33.572 & $3.71 \mathrm{E}-06$ & $3.1 \mathrm{E}+06$ \\
\hline 2 & 63 & 33.559 & $2.08 \mathrm{E}-04$ & $1.8 \mathrm{E}+08$ \\
\hline 2 & 64 & 33.558 & $1.49 \mathrm{E}-05$ & $1.8 \mathrm{E}+07$ \\
\hline 3 & 6 & 385.42 & $5.16 \mathrm{E}-03$ & $7.7 \mathrm{E}+07$ \\
\hline 3 & 7 & 348.05 & $2.65 \mathrm{E}-01$ & $2.9 \mathrm{E}+09$ \\
\hline 3 & 9 & 314.95 & $3.21 \mathrm{E}-01$ & $7.2 \mathrm{E}+09$ \\
\hline 3 & 10 & 297.88 & $2.70 \mathrm{E}-01$ & $4.1 \mathrm{E}+09$ \\
\hline 3 & 11 & 254.34 & $9.98 \mathrm{E}-02$ & $1.0 \mathrm{E}+10$ \\
\hline 3 & 12 & 235.72 & $9.08 \mathrm{E}-04$ & $3.6 \mathrm{E}+07$ \\
\hline 3 & 13 & 217.27 & $8.09 \mathrm{E}-05$ & $3.8 \mathrm{E}+06$ \\
\hline 3 & 14 & 215.17 & $2.32 \mathrm{E}-03$ & $6.7 \mathrm{E}+07$ \\
\hline 3 & 15 & 175.98 & $5.65 \mathrm{E}-02$ & $1.2 \mathrm{E}+10$ \\
\hline 3 & 30 & 38.712 & $4.06 \mathrm{E}-05$ & $6.0 \mathrm{E}+07$ \\
\hline 3 & 31 & 38.635 & $2.29 \mathrm{E}-01$ & $2.0 \mathrm{E}+11$ \\
\hline 3 & 33 & 38.46 & $2.60 \mathrm{E}-01$ & $3.9 \mathrm{E}+11$ \\
\hline 3 & 34 & 38.383 & $2.01 \mathrm{E}-01$ & $1.8 \mathrm{E}+11$ \\
\hline
\end{tabular}




\section{Continued}

\begin{tabular}{|c|c|c|c|c|}
\hline 3 & 37 & 37.914 & $7.33 \mathrm{E}-02$ & $3.4 \mathrm{E}+11$ \\
\hline 3 & 38 & 36.621 & $5.57 \mathrm{E}-04$ & $9.2 \mathrm{E}+08$ \\
\hline 3 & 39 & 36.444 & $3.26 \mathrm{E}-04$ & $5.4 \mathrm{E}+08$ \\
\hline 3 & 44 & 36.42 & $2.37 \mathrm{E}-03$ & $2.4 \mathrm{E}+09$ \\
\hline 3 & 48 & 36.204 & $4.06 \mathrm{E}-04$ & $2.1 \mathrm{E}+09$ \\
\hline 3 & 50 & 35.55 & $4.47 \mathrm{E}-05$ & $7.9 \mathrm{E}+07$ \\
\hline 3 & 51 & 35.528 & $2.14 \mathrm{E}-05$ & $2.3 \mathrm{E}+07$ \\
\hline 3 & 55 & 35.472 & $1.25 \mathrm{E}-04$ & $1.3 \mathrm{E}+08$ \\
\hline 3 & 64 & 33.754 & $1.23 \mathrm{E}-04$ & $1.4 \mathrm{E}+08$ \\
\hline 4 & 6 & 744.197 & $1.82 \mathrm{E}-03$ & $7.3 \mathrm{E}+06$ \\
\hline 4 & 9 & 519.673 & $2.30 \mathrm{E}-04$ & $1.9 \mathrm{E}+06$ \\
\hline 4 & 12 & 334.278 & $1.17 \mathrm{E}-01$ & $2.3 \mathrm{E}+09$ \\
\hline 4 & 13 & 298.361 & $2.11 \mathrm{E}-01$ & $5.3 \mathrm{E}+09$ \\
\hline 4 & 30 & 40.682 & $7.08 \mathrm{E}-04$ & $9.5 \mathrm{E}+08$ \\
\hline 4 & 33 & 40.404 & $5.55 \mathrm{E}-07$ & $7.6 \mathrm{E}+05$ \\
\hline 4 & 39 & 38.379 & $8.85 \mathrm{E}-02$ & $1.3 \mathrm{E}+11$ \\
\hline 4 & 44 & 38.184 & $1.66 \mathrm{E}-01$ & $2.5 \mathrm{E}+11$ \\
\hline 4 & 50 & 37.204 & $2.02 \mathrm{E}-04$ & $3.2 \mathrm{E}+08$ \\
\hline 5 & 6 & 807.511 & $2.34 \mathrm{E}-03$ & $8.0 \mathrm{E}+06$ \\
\hline 5 & 7 & 659.218 & $2.17 \mathrm{E}-08$ & $6.7 \mathrm{E}+01$ \\
\hline 5 & 9 & 549.774 & $1.78 \mathrm{E}-04$ & $1.3 \mathrm{E}+06$ \\
\hline 5 & 10 & 499.769 & $2.49 \mathrm{E}-03$ & $1.3 \mathrm{E}+07$ \\
\hline 5 & 11 & 388.268 & $2.31 \mathrm{E}-02$ & $1.0 \mathrm{E}+09$ \\
\hline 5 & 12 & 346.481 & $1.85 \mathrm{E}-01$ & $3.4 \mathrm{E}+09$ \\
\hline 5 & 13 & 308.044 & $1.22 \mathrm{E}-01$ & $2.8 \mathrm{E}+09$ \\
\hline 5 & 14 & 303.823 & $5.66 \mathrm{E}-01$ & $8.2 \mathrm{E}+09$ \\
\hline 5 & 15 & 231.146 & $1.13 \mathrm{E}-01$ & $1.4 \mathrm{E}+10$ \\
\hline 5 & 30 & 40.858 & $8.05 \mathrm{E}-04$ & $1.1 \mathrm{E}+09$ \\
\hline 5 & 31 & 40.772 & $1.06 \mathrm{E}-03$ & $8.5 \mathrm{E}+08$ \\
\hline 5 & 33 & 40.577 & $1.05 \mathrm{E}-03$ & $1.4 \mathrm{E}+09$ \\
\hline 5 & 34 & 40.49 & $2.93 \mathrm{E}-03$ & $2.4 \mathrm{E}+09$ \\
\hline 5 & 37 & 39.969 & $2.25 \mathrm{E}-03$ & $9.4 \mathrm{E}+09$ \\
\hline 5 & 38 & 38.535 & $1.68 \mathrm{E}-01$ & $2.5 \mathrm{E}+11$ \\
\hline 5 & 39 & 38.338 & $8.95 \mathrm{E}-02$ & $1.4 \mathrm{E}+11$ \\
\hline 5 & 44 & 38.312 & $4.28 \mathrm{E}-01$ & $3.9 \mathrm{E}+11$ \\
\hline 5 & 48 & 38.074 & 7.35E-02 & $3.4 \mathrm{E}+11$ \\
\hline 5 & 50 & 37.351 & $8.91 \mathrm{E}-05$ & $1.4 \mathrm{E}+08$ \\
\hline 5 & 51 & 37.326 & $3.94 \mathrm{E}-04$ & $3.8 \mathrm{E}+08$ \\
\hline 5 & 55 & 37.265 & $5.09 \mathrm{E}-05$ & $4.9 \mathrm{E}+07$ \\
\hline 5 & 64 & 35.373 & $8.26 \mathrm{E}-08$ & $8.8 \mathrm{E}+04$ \\
\hline 6 & 16 & 240.468 & $1.25 \mathrm{E}-01$ & $1.4 \mathrm{E}+10$ \\
\hline 6 & 17 & 234.884 & $2.92 \mathrm{E}-01$ & $1.2 \mathrm{E}+10$ \\
\hline 6 & 19 & 225.329 & $2.34 \mathrm{E}-01$ & $6.1 \mathrm{E}+09$ \\
\hline 6 & 21 & 215.032 & $2.11 \mathrm{E}-02$ & $6.1 \mathrm{E}+08$ \\
\hline 6 & 23 & 192.547 & $2.96 \mathrm{E}-03$ & $1.8 \mathrm{E}+08$ \\
\hline 6 & 24 & 169.37 & $5.86 \mathrm{E}-04$ & $2.7 \mathrm{E}+07$ \\
\hline 6 & 25 & 166.964 & $2.37 \mathrm{E}-03$ & $1.1 \mathrm{E}+08$ \\
\hline 6 & 27 & 151.559 & $3.85 \mathrm{E}-07$ & $3.7 \mathrm{E}+04$ \\
\hline 6 & 28 & 45.347 & $1.66 \mathrm{E}-01$ & $1.1 \mathrm{E}+11$ \\
\hline
\end{tabular}




\section{Continued}

\begin{tabular}{|c|c|c|c|c|}
\hline 6 & 29 & 45.347 & $2.22 \mathrm{E}-03$ & $2.4 \mathrm{E}+09$ \\
\hline 6 & 35 & 42.625 & $2.13 \mathrm{E}-03$ & $7.8 \mathrm{E}+09$ \\
\hline 6 & 36 & 42.625 & $3.63 \mathrm{E}-03$ & $4.4 \mathrm{E}+09$ \\
\hline 6 & 38 & 40.491 & $2.12 \mathrm{E}-01$ & $8.6 \mathrm{E}+11$ \\
\hline 6 & 40 & 40.412 & $4.76 \mathrm{E}-01$ & $6.5 \mathrm{E}+11$ \\
\hline 6 & 43 & 40.267 & $3.74 \mathrm{E}-01$ & $3.1 \mathrm{E}+11$ \\
\hline 6 & 47 & 40.081 & $1.59 \mathrm{E}-02$ & $1.3 \mathrm{E}+10$ \\
\hline 6 & 49 & 39.86 & $5.89 \mathrm{E}-03$ & $8.2 \mathrm{E}+09$ \\
\hline 6 & 58 & 38.104 & $5.28 \mathrm{E}-04$ & $4.9 \mathrm{E}+08$ \\
\hline 6 & 60 & 38.049 & $1.14 \mathrm{E}-02$ & $1.0 \mathrm{E}+10$ \\
\hline 6 & 61 & 37.868 & $9.27 \mathrm{E}-04$ & $1.4 \mathrm{E}+09$ \\
\hline 7 & 17 & 251.329 & $3.53 \mathrm{E}-02$ & $1.2 \mathrm{E}+09$ \\
\hline 7 & 19 & 240.42 & $5.82 \mathrm{E}-02$ & $1.3 \mathrm{E}+09$ \\
\hline 7 & 20 & 237.41 & $7.91 \mathrm{E}-01$ & $1.3 \mathrm{E}+10$ \\
\hline 7 & 21 & 228.73 & $2.20 \mathrm{E}-01$ & $5.6 \mathrm{E}+09$ \\
\hline 7 & 22 & 223.34 & $5.66 \mathrm{E}-03$ & $1.1 \mathrm{E}+08$ \\
\hline 7 & 23 & 203.46 & $1.63 \mathrm{E}-02$ & $8.7 \mathrm{E}+08$ \\
\hline 7 & 24 & 177.76 & $1.64 \mathrm{E}-03$ & $6.9 \mathrm{E}+07$ \\
\hline 7 & 25 & 175.11 & $4.55 \mathrm{E}-03$ & $2.0 \mathrm{E}+08$ \\
\hline 7 & 26 & 173.41 & $2.39 \mathrm{E}-04$ & $7.6 \mathrm{E}+06$ \\
\hline 7 & 27 & 158.24 & $4.06 \mathrm{E}-05$ & $3.6 \mathrm{E}+06$ \\
\hline 7 & 28 & 45.928 & $1.39 \mathrm{E}-01$ & $8.8 \mathrm{E}+10$ \\
\hline 7 & 29 & 45.927 & $1.56 \mathrm{E}-01$ & $1.6 \mathrm{E}+11$ \\
\hline 7 & 36 & 43.137 & $1.37 \mathrm{E}-03$ & $1.6 \mathrm{E}+09$ \\
\hline 7 & 40 & 40.873 & $6.47 \mathrm{E}-02$ & $8.6 \mathrm{E}+10$ \\
\hline 7 & 42 & 40.771 & $1.29 \mathrm{E}+00$ & $7.4 \mathrm{E}+11$ \\
\hline 7 & 43 & 40.724 & $1.36 \mathrm{E}-01$ & $1.1 \mathrm{E}+11$ \\
\hline 7 & 46 & 40.556 & $3.23 E-02$ & $1.9 \mathrm{E}+10$ \\
\hline 7 & 47 & 40.534 & $3.18 \mathrm{E}-01$ & $2.6 \mathrm{E}+11$ \\
\hline 7 & 49 & 40.308 & $2.24 \mathrm{E}-02$ & $3.1 \mathrm{E}+10$ \\
\hline 7 & 58 & 38.513 & $7.19 \mathrm{E}-04$ & $6.5 \mathrm{E}+08$ \\
\hline 7 & 59 & 38.483 & $3.22 \mathrm{E}-03$ & $2.1 \mathrm{E}+09$ \\
\hline 7 & 60 & 38.457 & $1.05 \mathrm{E}-03$ & $9.5 \mathrm{E}+08$ \\
\hline 7 & 61 & 38.271 & $2.99 \mathrm{E}-04$ & $4.5 \mathrm{E}+08$ \\
\hline 8 & 18 & 254.11 & $1.13 \mathrm{E}+00$ & $1.3 \mathrm{E}+10$ \\
\hline 8 & 19 & 253.76 & $2.90 \mathrm{E}-02$ & $6.0 \mathrm{E}+08$ \\
\hline 8 & 20 & 250.42 & $1.33 \mathrm{E}-01$ & $2.0 \mathrm{E}+09$ \\
\hline 8 & 21 & 240.78 & $9.38 \mathrm{E}-03$ & $2.2 \mathrm{E}+08$ \\
\hline 8 & 22 & 234.81 & $1.73 \mathrm{E}-01$ & $3.0 \mathrm{E}+09$ \\
\hline 8 & 24 & 184.95 & $1.16 \mathrm{E}-04$ & $4.5 \mathrm{E}+06$ \\
\hline 8 & 25 & 182.08 & $2.06 \mathrm{E}-03$ & $8.3 \mathrm{E}+07$ \\
\hline 8 & 26 & 180.24 & $3.85 \mathrm{E}-03$ & $1.1 \mathrm{E}+08$ \\
\hline 8 & 28 & 46.394 & $4.06 \mathrm{E}-01$ & $2.5 \mathrm{E}+11$ \\
\hline 8 & 41 & 41.151 & $1.99 \mathrm{E}+00$ & $8.7 \mathrm{E}+11$ \\
\hline 8 & 42 & 41.137 & $1.20 \mathrm{E}-01$ & $6.7 \mathrm{E}+10$ \\
\hline 8 & 43 & 41.09 & $6.08 \mathrm{E}-02$ & $4.8 \mathrm{E}+10$ \\
\hline 8 & 46 & 40.919 & $3.89 \mathrm{E}-01$ & $2.2 \mathrm{E}+11$ \\
\hline 8 & 47 & 40.896 & $1.03 E-02$ & $8.2 \mathrm{E}+09$ \\
\hline 8 & 58 & 38.84 & $1.70 \mathrm{E}-04$ & $1.5 \mathrm{E}+08$ \\
\hline
\end{tabular}




\section{Continued}

\begin{tabular}{|c|c|c|c|c|}
\hline 8 & 59 & 38.81 & $1.11 \mathrm{E}-03$ & $7.0 \mathrm{E}+08$ \\
\hline 8 & 60 & 38.783 & $3.24 \mathrm{E}-04$ & $2.9 \mathrm{E}+08$ \\
\hline 9 & 16 & 279.49 & $2.10 \mathrm{E}-03$ & $1.8 \mathrm{E}+08$ \\
\hline 9 & 17 & 271.97 & $7.38 \mathrm{E}-07$ & $2.2 \mathrm{E}+04$ \\
\hline 9 & 19 & 259.24 & $6.35 E-02$ & $1.3 \mathrm{E}+09$ \\
\hline 9 & 21 & 245.71 & $4.17 \mathrm{E}-01$ & $9.2 \mathrm{E}+09$ \\
\hline 9 & 23 & 216.78 & $1.81 \mathrm{E}-01$ & $8.5 \mathrm{E}+09$ \\
\hline 9 & 24 & 187.84 & $1.65 \mathrm{E}-05$ & $6.2 \mathrm{E}+05$ \\
\hline 9 & 25 & 184.89 & $3.38 \mathrm{E}-04$ & $1.3 \mathrm{E}+07$ \\
\hline 9 & 27 & 166.18 & $1.69 \mathrm{E}-02$ & $1.4 \mathrm{E}+09$ \\
\hline 9 & 28 & 46.573 & $3.71 \mathrm{E}-03$ & $2.3 \mathrm{E}+09$ \\
\hline 9 & 29 & 46.573 & $1.66 \mathrm{E}-01$ & $1.7 \mathrm{E}+11$ \\
\hline 9 & 35 & 43.706 & $2.01 \mathrm{E}-04$ & $7.0 \mathrm{E}+08$ \\
\hline 9 & 36 & 43.706 & $3.03 \mathrm{E}-03$ & $3.5 \mathrm{E}+09$ \\
\hline 9 & 38 & 41.465 & $4.75 E-03$ & $1.8 \mathrm{E}+10$ \\
\hline 9 & 40 & 41.383 & $2.04 \mathrm{E}-05$ & $2.6 \mathrm{E}+07$ \\
\hline 9 & 43 & 41.231 & $6.70 \mathrm{E}-02$ & $5.3 \mathrm{E}+10$ \\
\hline 9 & 47 & 41.036 & $7.60 \mathrm{E}-01$ & $6.0 \mathrm{E}+11$ \\
\hline 9 & 49 & 40.804 & $2.64 \mathrm{E}-01$ & $3.5 \mathrm{E}+11$ \\
\hline 9 & 58 & 38.966 & $1.23 \mathrm{E}-03$ & $1.1 \mathrm{E}+09$ \\
\hline 9 & 60 & 38.909 & $4.23 \mathrm{E}-04$ & $3.7 \mathrm{E}+08$ \\
\hline 9 & 61 & 38.719 & $1.07 \mathrm{E}-03$ & $1.6 \mathrm{E}+09$ \\
\hline 10 & 17 & 286.133 & $4.79 \mathrm{E}-02$ & $1.3 \mathrm{E}+09$ \\
\hline 10 & 19 & 272.079 & $2.44 \mathrm{E}-01$ & $4.4 \mathrm{E}+09$ \\
\hline 10 & 20 & 268.235 & $9.77 \mathrm{E}-03$ & $1.3 \mathrm{E}+08$ \\
\hline 10 & 21 & 257.206 & $1.55 \mathrm{E}-03$ & $3.1 \mathrm{E}+07$ \\
\hline 10 & 22 & 250.403 & $7.29 \mathrm{E}-01$ & $1.1 \mathrm{E}+10$ \\
\hline 10 & 23 & 225.684 & $2.45 \mathrm{E}-04$ & $1.1 \mathrm{E}+07$ \\
\hline 10 & 24 & 194.488 & $2.82 \mathrm{E}-04$ & $9.9 \mathrm{E}+06$ \\
\hline 10 & 25 & 191.323 & $1.34 \mathrm{E}-02$ & $4.9 \mathrm{E}+08$ \\
\hline 10 & 26 & 189.291 & $2.73 E-04$ & $7.3 \mathrm{E}+06$ \\
\hline 10 & 27 & 171.363 & $2.24 \mathrm{E}-03$ & $1.7 \mathrm{E}+08$ \\
\hline 10 & 28 & 46.972 & $1.56 \mathrm{E}-01$ & $9.4 \mathrm{E}+10$ \\
\hline 10 & 29 & 46.971 & $1.39 \mathrm{E}-01$ & $1.4 \mathrm{E}+11$ \\
\hline 10 & 36 & 44.057 & $9.33 \mathrm{E}-05$ & $1.1 \mathrm{E}+08$ \\
\hline 10 & 40 & 41.697 & $9.82 \mathrm{E}-02$ & $1.3 \mathrm{E}+11$ \\
\hline 10 & 42 & 41.591 & $1.55 \mathrm{E}-01$ & $8.5 E+10$ \\
\hline 10 & 43 & 41.543 & $4.67 \mathrm{E}-01$ & $3.6 \mathrm{E}+11$ \\
\hline 10 & 46 & 41.368 & $1.13 \mathrm{E}+00$ & $6.3 \mathrm{E}+11$ \\
\hline 10 & 47 & 41.345 & $1.36 \mathrm{E}-02$ & $1.1 \mathrm{E}+10$ \\
\hline 10 & 49 & 41.11 & $6.68 \mathrm{E}-04$ & $8.8 \mathrm{E}+08$ \\
\hline 10 & 58 & 39.245 & $8.53 E-05$ & $7.4 \mathrm{E}+07$ \\
\hline 10 & 59 & 39.213 & $6.50 \mathrm{E}-04$ & $4.0 \mathrm{E}+08$ \\
\hline 10 & 60 & 39.186 & $4.84 \mathrm{E}-03$ & $4.2 \mathrm{E}+09$ \\
\hline 10 & 61 & 38.994 & 4.33E-04 & $6.3 \mathrm{E}+08$ \\
\hline 11 & 17 & 342.436 & $8.02 E-03$ & $1.5 \mathrm{E}+08$ \\
\hline 11 & 23 & 259.312 & $1.85 \mathrm{E}-01$ & $6.1 \mathrm{E}+09$ \\
\hline 11 & 27 & 190.08 & $1.79 \mathrm{E}-02$ & $1.1 \mathrm{E}+09$ \\
\hline 11 & 29 & 48.274 & $4.48 \mathrm{E}-02$ & $4.3 \mathrm{E}+10$ \\
\hline
\end{tabular}




\section{Continued}

\begin{tabular}{|c|c|c|c|c|}
\hline 11 & 36 & 45.201 & $1.77 \mathrm{E}-02$ & $1.9 \mathrm{E}+10$ \\
\hline 11 & 40 & 42.721 & $1.75 \mathrm{E}-02$ & $2.1 \mathrm{E}+10$ \\
\hline 11 & 49 & 42.104 & $2.68 \mathrm{E}-01$ & $3.4 \mathrm{E}+11$ \\
\hline 11 & 61 & 39.887 & $9.73 \mathrm{E}-02$ & $1.4 \mathrm{E}+11$ \\
\hline 12 & 16 & 398.283 & $1.64 \mathrm{E}-05$ & $6.9 \mathrm{E}+05$ \\
\hline 12 & 17 & 383.195 & $1.02 \mathrm{E}-04$ & $1.5 \mathrm{E}+06$ \\
\hline 12 & 19 & 358.401 & $9.23 \mathrm{E}-04$ & $9.6 \mathrm{E}+06$ \\
\hline 12 & 21 & 333.035 & $2.92 \mathrm{E}-04$ & $3.5 \mathrm{E}+06$ \\
\hline 12 & 23 & 282.029 & $3.61 \mathrm{E}-03$ & $1.0 \mathrm{E}+08$ \\
\hline 12 & 24 & 234.937 & $5.70 \mathrm{E}-01$ & $1.4 \mathrm{E}+10$ \\
\hline 12 & 25 & 230.334 & $1.77 \mathrm{E}-03$ & $4.4 \mathrm{E}+07$ \\
\hline 12 & 27 & 202.007 & $1.22 \mathrm{E}-01$ & $6.6 \mathrm{E}+09$ \\
\hline 12 & 28 & 49.009 & $1.60 \mathrm{E}-04$ & $8.9 \mathrm{E}+07$ \\
\hline 12 & 29 & 49.009 & $2.55 \mathrm{E}-03$ & $2.4 \mathrm{E}+09$ \\
\hline 12 & 35 & 45.845 & 6.67E-02 & $2.1 \mathrm{E}+11$ \\
\hline 12 & 36 & 45.845 & $1.06 \mathrm{E}-01$ & $1.1 \mathrm{E}+11$ \\
\hline 12 & 38 & 43.385 & $1.01 \mathrm{E}-04$ & $3.6 \mathrm{E}+08$ \\
\hline 12 & 40 & 43.296 & $2.52 \mathrm{E}-05$ & $3.0 \mathrm{E}+07$ \\
\hline 12 & 43 & 43.129 & $2.78 \mathrm{E}-03$ & $2.0 \mathrm{E}+09$ \\
\hline 12 & 47 & 42.916 & 7.33E-04 & $5.3 \mathrm{E}+08$ \\
\hline 12 & 49 & 42.662 & $3.24 \mathrm{E}-04$ & $4.0 \mathrm{E}+08$ \\
\hline 12 & 58 & 40.657 & $7.83 \mathrm{E}-01$ & $6.3 \mathrm{E}+11$ \\
\hline 12 & 60 & 40.594 & $1.60 \mathrm{E}-01$ & $1.3 \mathrm{E}+11$ \\
\hline 12 & 61 & 40.388 & $1.64 \mathrm{E}-01$ & $2.2 \mathrm{E}+11$ \\
\hline 13 & 16 & 464.98 & $2.31 E-03$ & $7.1 \mathrm{E}+07$ \\
\hline 13 & 17 & 444.54 & $2.25 \mathrm{E}-03$ & $2.5 \mathrm{E}+07$ \\
\hline 13 & 19 & 411.52 & $1.25 \mathrm{E}-03$ & $9.9 \mathrm{E}+06$ \\
\hline 13 & 21 & 378.42 & $1.86 \mathrm{E}-04$ & $1.7 \mathrm{E}+06$ \\
\hline 13 & 23 & 313.91 & 4.33E-03 & $9.8 \mathrm{E}+07$ \\
\hline 13 & 24 & 256.65 & $2.01 \mathrm{E}-02$ & $4.1 \mathrm{E}+08$ \\
\hline 13 & 25 & 251.17 & $5.46 \mathrm{E}-01$ & $1.2 \mathrm{E}+10$ \\
\hline 13 & 27 & 217.86 & $6.70 \mathrm{E}-02$ & $3.1 \mathrm{E}+09$ \\
\hline 13 & 28 & 49.89 & $5.55 \mathrm{E}-03$ & $3.0 \mathrm{E}+09$ \\
\hline 13 & 29 & 49.889 & $8.32 \mathrm{E}-04$ & $7.4 \mathrm{E}+08$ \\
\hline 13 & 35 & 46.615 & $1.06 \mathrm{E}-01$ & $3.3 \mathrm{E}+11$ \\
\hline 13 & 36 & 46.614 & $6.15 \mathrm{E}-02$ & $6.3 \mathrm{E}+10$ \\
\hline 13 & 38 & 44.074 & $7.85 \mathrm{E}-03$ & $2.7 \mathrm{E}+10$ \\
\hline 13 & 40 & 43.981 & $1.16 \mathrm{E}-02$ & $1.3 \mathrm{E}+10$ \\
\hline 13 & 43 & 43.809 & $7.36 \mathrm{E}-04$ & $5.1 \mathrm{E}+08$ \\
\hline 13 & 47 & 43.589 & $8.65 \mathrm{E}-05$ & $6.1 \mathrm{E}+07$ \\
\hline 13 & 49 & 43.328 & $3.95 \mathrm{E}-04$ & $4.7 \mathrm{E}+08$ \\
\hline 13 & 58 & 41.261 & $2.83 \mathrm{E}-01$ & $2.2 \mathrm{E}+11$ \\
\hline 13 & 60 & 41.196 & $7.06 \mathrm{E}-01$ & $5.6 \mathrm{E}+11$ \\
\hline 13 & 61 & 40.984 & $9.86 \mathrm{E}-02$ & $1.3 \mathrm{E}+11$ \\
\hline 14 & 17 & 453.64 & $8.65 \mathrm{E}-04$ & $9.3 \mathrm{E}+06$ \\
\hline 14 & 19 & 419.3 & $3.81 \mathrm{E}-03$ & $2.9 \mathrm{E}+07$ \\
\hline 14 & 20 & 410.24 & $4.24 \mathrm{E}-04$ & $2.4 \mathrm{E}+06$ \\
\hline 14 & 21 & 384.99 & $3.05 \mathrm{E}-05$ & $2.7 \mathrm{E}+05$ \\
\hline 14 & 22 & 369.95 & $7.78 \mathrm{E}-04$ & $5.4 \mathrm{E}+06$ \\
\hline
\end{tabular}




\section{Continued}

\begin{tabular}{|c|c|c|c|c|}
\hline 14 & 23 & 318.42 & $8.63 \mathrm{E}-04$ & $1.9 \mathrm{E}+07$ \\
\hline 14 & 24 & 259.66 & $7.76 \mathrm{E}-02$ & $1.5 \mathrm{E}+09$ \\
\hline 14 & 25 & 254.05 & $7.23 \mathrm{E}-02$ & $1.5 \mathrm{E}+09$ \\
\hline 14 & 26 & 250.48 & $8.91 \mathrm{E}-01$ & $1.4 \mathrm{E}+10$ \\
\hline 14 & 27 & 220.02 & $1.00 \mathrm{E}-02$ & $4.6 \mathrm{E}+08$ \\
\hline 14 & 28 & 50.003 & $1.22 \mathrm{E}-03$ & $6.5 \mathrm{E}+08$ \\
\hline 14 & 29 & 50.002 & $5.53 \mathrm{E}-04$ & $4.9 \mathrm{E}+08$ \\
\hline 14 & 36 & 46.712 & $2.90 \mathrm{E}-01$ & $3.0 \mathrm{E}+11$ \\
\hline 14 & 40 & 44.069 & $1.78 \mathrm{E}-03$ & $2.0 \mathrm{E}+09$ \\
\hline 14 & 42 & 43.95 & $2.86 \mathrm{E}-03$ & $1.4 \mathrm{E}+09$ \\
\hline 14 & 43 & 43.896 & $6.14 \mathrm{E}-03$ & $4.3 \mathrm{E}+09$ \\
\hline 14 & 46 & 43.701 & $1.04 \mathrm{E}-03$ & $5.2 \mathrm{E}+08$ \\
\hline 14 & 47 & 43.675 & $1.01 \mathrm{E}-05$ & $7.1 \mathrm{E}+06$ \\
\hline 14 & 49 & 43.413 & $4.70 \mathrm{E}-05$ & $5.6 \mathrm{E}+07$ \\
\hline 14 & 58 & 41.338 & $4.69 \mathrm{E}-02$ & $3.7 \mathrm{E}+10$ \\
\hline 14 & 59 & 41.304 & $1.55 \mathrm{E}+00$ & $8.6 \mathrm{E}+11$ \\
\hline 14 & 60 & 41.273 & $2.29 \mathrm{E}-01$ & $1.8 \mathrm{E}+11$ \\
\hline 14 & 61 & 41.06 & $1.70 \mathrm{E}-02$ & $2.2 \mathrm{E}+10$ \\
\hline 15 & 17 & 855.04 & $2.96 \mathrm{E}-06$ & $9.0 \mathrm{E}+03$ \\
\hline 15 & 23 & 474.92 & $7.94 \mathrm{E}-03$ & $7.8 \mathrm{E}+07$ \\
\hline 15 & 27 & 284.88 & $1.78 \mathrm{E}-01$ & $4.9 \mathrm{E}+09$ \\
\hline 15 & 29 & 52.73 & $3.90 \mathrm{E}-02$ & $3.1 \mathrm{E}+10$ \\
\hline 15 & 36 & 49.085 & $5.47 \mathrm{E}-02$ & $5.0 \mathrm{E}+10$ \\
\hline 15 & 40 & 46.175 & $1.72 \mathrm{E}-03$ & $1.8 \mathrm{E}+09$ \\
\hline 15 & 49 & 45.455 & $1.25 \mathrm{E}-01$ & $1.3 \mathrm{E}+11$ \\
\hline 15 & 61 & 42.882 & $3.08 \mathrm{E}-01$ & $3.7 \mathrm{E}+11$ \\
\hline 16 & 30 & 52.415 & $2.84 \mathrm{E}-02$ & $2.3 E+10$ \\
\hline 16 & 33 & 51.954 & $6.67 \mathrm{E}-06$ & $5.5 \mathrm{E}+06$ \\
\hline 16 & 39 & 48.653 & $2.21 \mathrm{E}-05$ & $2.1 \mathrm{E}+07$ \\
\hline 16 & 44 & 48.341 & $1.96 \mathrm{E}-04$ & $1.9 \mathrm{E}+08$ \\
\hline 16 & 50 & 46.781 & $9.33 \mathrm{E}-01$ & $9.5 E+11$ \\
\hline 17 & 30 & 52.688 & $6.32 \mathrm{E}-02$ & $5.1 \mathrm{E}+10$ \\
\hline 17 & 31 & 52.546 & $7.59 \mathrm{E}-03$ & $3.7 \mathrm{E}+09$ \\
\hline 17 & 33 & 52.222 & $1.10 \mathrm{E}-03$ & $8.9 \mathrm{E}+08$ \\
\hline 17 & 34 & 52.08 & $1.26 \mathrm{E}-02$ & $6.2 \mathrm{E}+09$ \\
\hline 17 & 37 & 51.22 & $1.91 \mathrm{E}-03$ & $4.9 \mathrm{E}+09$ \\
\hline 17 & 38 & 48.888 & $4.29 \mathrm{E}-05$ & $4.0 \mathrm{E}+07$ \\
\hline 17 & 39 & 48.573 & $6.21 \mathrm{E}-06$ & $5.9 \mathrm{E}+06$ \\
\hline 17 & 44 & 48.531 & $2.94 \mathrm{E}-04$ & $1.7 \mathrm{E}+08$ \\
\hline 17 & 48 & 48.149 & $3.51 \mathrm{E}-04$ & $1.0 \mathrm{E}+09$ \\
\hline 17 & 50 & 46.998 & $8.89 \mathrm{E}-01$ & $9.0 \mathrm{E}+11$ \\
\hline 17 & 51 & 46.959 & $1.68 \mathrm{E}+00$ & $1.0 \mathrm{E}+12$ \\
\hline 17 & 55 & 46.862 & $1.94 \mathrm{E}-01$ & $1.2 \mathrm{E}+11$ \\
\hline 17 & 64 & 43.909 & $3.77 \mathrm{E}-02$ & $2.6 \mathrm{E}+10$ \\
\hline 18 & 32 & 52.773 & $2.70 \mathrm{E}-01$ & $9.2 \mathrm{E}+10$ \\
\hline 18 & 52 & 47.328 & $2.35 E-01$ & $7.8 \mathrm{E}+10$ \\
\hline 18 & 53 & 47.317 & $7.36 \mathrm{E}+00$ & $2.0 \mathrm{E}+12$ \\
\hline 18 & 54 & 47.265 & $5.38 \mathrm{E}-02$ & $2.3 \mathrm{E}+10$ \\
\hline 18 & 56 & 47.23 & $1.31 \mathrm{E}-02$ & $5.6 \mathrm{E}+09$ \\
\hline
\end{tabular}




\section{Continued}

\begin{tabular}{|c|c|c|c|c|}
\hline 18 & 57 & 47.209 & $7.69 \mathrm{E}-01$ & $2.6 \mathrm{E}+11$ \\
\hline 18 & 62 & 44.272 & $5.07 \mathrm{E}-06$ & $2.5 \mathrm{E}+06$ \\
\hline 18 & 63 & 44.251 & $4.46 \mathrm{E}-05$ & $2.2 \mathrm{E}+07$ \\
\hline 18 & 65 & 44.247 & $3.37 \mathrm{E}-04$ & $1.3 \mathrm{E}+08$ \\
\hline 19 & 30 & 53.194 & $4.04 \mathrm{E}-02$ & $3.2 \mathrm{E}+10$ \\
\hline 19 & 31 & 53.049 & $1.46 \mathrm{E}-02$ & $6.9 \mathrm{E}+09$ \\
\hline 19 & 32 & 52.788 & $5.93 \mathrm{E}-03$ & $2.0 \mathrm{E}+09$ \\
\hline 19 & 33 & 52.719 & $2.32 \mathrm{E}-02$ & $1.9 \mathrm{E}+10$ \\
\hline 19 & 34 & 52.574 & $6.27 \mathrm{E}-02$ & $3.0 \mathrm{E}+10$ \\
\hline 19 & 39 & 49.323 & $8.11 \mathrm{E}-06$ & $7.4 \mathrm{E}+06$ \\
\hline 19 & 44 & 49.002 & $1.95 \mathrm{E}-03$ & $1.8 \mathrm{E}+09$ \\
\hline 19 & 45 & 48.96 & $5.82 \mathrm{E}-04$ & $3.2 \mathrm{E}+08$ \\
\hline 19 & 50 & 47.4 & $1.02 \mathrm{E}-01$ & $1.0 \mathrm{E}+11$ \\
\hline 19 & 51 & 47.361 & $1.07 \mathrm{E}+00$ & $6.4 \mathrm{E}+11$ \\
\hline 19 & 54 & 47.277 & $3.32 \mathrm{E}+00$ & $1.4 \mathrm{E}+12$ \\
\hline 19 & 55 & 47.262 & $6.04 \mathrm{E}-02$ & $3.6 \mathrm{E}+10$ \\
\hline 19 & 56 & 47.242 & $1.55 \mathrm{E}-02$ & $6.6 \mathrm{E}+09$ \\
\hline 19 & 62 & 44.283 & $8.99 \mathrm{E}-04$ & $4.4 \mathrm{E}+08$ \\
\hline 19 & 63 & 44.261 & $1.12 \mathrm{E}-01$ & $5.5 \mathrm{E}+10$ \\
\hline 19 & 64 & 44.26 & $6.30 \mathrm{E}-03$ & $4.3 \mathrm{E}+09$ \\
\hline 20 & 31 & 53.197 & $1.79 \mathrm{E}-01$ & $8.5 \mathrm{E}+10$ \\
\hline 20 & 32 & 52.935 & $3.26 \mathrm{E}-02$ & $1.1 \mathrm{E}+10$ \\
\hline 20 & 34 & 52.72 & $2.79 \mathrm{E}-03$ & $1.3 \mathrm{E}+09$ \\
\hline 20 & 45 & 49.087 & $6.59 \mathrm{E}-04$ & $3.7 \mathrm{E}+08$ \\
\hline 20 & 51 & 47.479 & $2.69 \mathrm{E}-02$ & $1.6 \mathrm{E}+10$ \\
\hline 20 & 52 & 47.458 & $5.37 \mathrm{E}+00$ & $1.8 \mathrm{E}+12$ \\
\hline 20 & 54 & 47.395 & $2.26 \mathrm{E}-01$ & $9.6 \mathrm{E}+10$ \\
\hline 20 & 55 & 47.38 & $4.14 \mathrm{E}-02$ & $2.5 \mathrm{E}+10$ \\
\hline 20 & 56 & 47.36 & $6.93 \mathrm{E}-01$ & $2.9 \mathrm{E}+11$ \\
\hline 20 & 57 & 47.338 & $1.94 \mathrm{E}-01$ & $6.4 \mathrm{E}+10$ \\
\hline 20 & 62 & 44.386 & $1.04 \mathrm{E}-03$ & $5.0 \mathrm{E}+08$ \\
\hline 20 & 63 & 44.365 & $3.07 \mathrm{E}-03$ & $1.5 \mathrm{E}+09$ \\
\hline 20 & 64 & 44.363 & $3.07 \mathrm{E}-04$ & $2.1 \mathrm{E}+08$ \\
\hline 20 & 65 & 44.361 & $4.19 \mathrm{E}-02$ & $1.6 \mathrm{E}+10$ \\
\hline 21 & 30 & 53.803 & $1.17 \mathrm{E}-02$ & $9.0 \mathrm{E}+09$ \\
\hline 21 & 31 & 53.654 & $4.98 \mathrm{E}-02$ & $2.3 \mathrm{E}+10$ \\
\hline 21 & 32 & 53.387 & $2.41 \mathrm{E}-03$ & $8.0 \mathrm{E}+08$ \\
\hline 21 & 33 & 53.317 & $9.23 \mathrm{E}-02$ & $7.2 \mathrm{E}+10$ \\
\hline 21 & 34 & 53.168 & $3.81 \mathrm{E}-04$ & $1.8 \mathrm{E}+08$ \\
\hline 21 & 39 & 49.846 & $9.27 \mathrm{E}-04$ & $8.3 \mathrm{E}+08$ \\
\hline 21 & 44 & 49.518 & $1.92 \mathrm{E}-05$ & $1.7 \mathrm{E}+07$ \\
\hline 21 & 45 & 49.475 & $1.54 \mathrm{E}-04$ & $8.4 \mathrm{E}+07$ \\
\hline 21 & 50 & 47.882 & $2.80 \mathrm{E}-02$ & $2.7 \mathrm{E}+10$ \\
\hline 21 & 51 & 47.842 & $1.56 \mathrm{E}-02$ & $9.1 \mathrm{E}+09$ \\
\hline 21 & 54 & 47.757 & $4.04 \mathrm{E}-03$ & $1.7 \mathrm{E}+09$ \\
\hline 21 & 55 & 47.741 & $6.70 \mathrm{E}-01$ & $3.9 \mathrm{E}+11$ \\
\hline 21 & 56 & 47.721 & $3.94 \mathrm{E}+00$ & $1.7 \mathrm{E}+12$ \\
\hline 21 & 62 & 44.704 & $4.66 \mathrm{E}-02$ & $2.2 \mathrm{E}+10$ \\
\hline 21 & 63 & 44.682 & $5.77 \mathrm{E}-06$ & $2.8 \mathrm{E}+06$ \\
\hline
\end{tabular}




\section{Continued}

\begin{tabular}{|c|c|c|c|c|}
\hline 21 & 64 & 44.68 & $7.50 \mathrm{E}-03$ & $5.0 \mathrm{E}+09$ \\
\hline 22 & 31 & 53.96 & $1.40 \mathrm{E}-03$ & $6.4 \mathrm{E}+08$ \\
\hline 22 & 32 & 53.69 & $4.20 \mathrm{E}-02$ & $1.4 \mathrm{E}+10$ \\
\hline 22 & 34 & 53.468 & $1.83 \mathrm{E}-01$ & $8.6 \mathrm{E}+10$ \\
\hline 22 & 45 & 49.735 & $3.05 \mathrm{E}-04$ & $1.6 \mathrm{E}+08$ \\
\hline 22 & 51 & 48.085 & $7.52 \mathrm{E}-02$ & $4.3 \mathrm{E}+10$ \\
\hline 22 & 52 & 48.063 & $4.29 \mathrm{E}-01$ & $1.4 \mathrm{E}+11$ \\
\hline 22 & 54 & 47.999 & $1.00 \mathrm{E}+00$ & $4.2 \mathrm{E}+11$ \\
\hline 22 & 55 & 47.983 & $4.62 \mathrm{E}-03$ & $2.7 \mathrm{E}+09$ \\
\hline 22 & 56 & 47.963 & $2.51 \mathrm{E}-03$ & $1.0 \mathrm{E}+09$ \\
\hline 22 & 57 & 47.941 & $5.11 \mathrm{E}+00$ & $1.6 \mathrm{E}+12$ \\
\hline 22 & 62 & 44.916 & $6.43 \mathrm{E}-04$ & $3.0 \mathrm{E}+08$ \\
\hline 22 & 63 & 44.893 & $2.67 \mathrm{E}-03$ & $1.3 \mathrm{E}+09$ \\
\hline 22 & 64 & 44.892 & $1.22 \mathrm{E}-04$ & $8.1 \mathrm{E}+07$ \\
\hline 22 & 65 & 44.889 & $4.88 \mathrm{E}-03$ & $1.8 \mathrm{E}+09$ \\
\hline 23 & 30 & 55.422 & $3.44 \mathrm{E}-03$ & $2.5 E+09$ \\
\hline 23 & 31 & 55.264 & $3.75 \mathrm{E}-03$ & $1.6 \mathrm{E}+09$ \\
\hline 23 & 33 & 54.906 & $4.42 \mathrm{E}-02$ & $3.3 \mathrm{E}+10$ \\
\hline 23 & 34 & 54.749 & $6.35 \mathrm{E}-05$ & $2.8 \mathrm{E}+07$ \\
\hline 23 & 37 & 53.8 & $5.30 \mathrm{E}-02$ & $1.2 \mathrm{E}+11$ \\
\hline 23 & 38 & 51.232 & $1.34 \mathrm{E}-03$ & $1.1 \mathrm{E}+09$ \\
\hline 23 & 39 & 50.886 & $8.47 \mathrm{E}-04$ & $7.3 E+08$ \\
\hline 23 & 44 & 50.841 & $3.74 \mathrm{E}-04$ & $1.9 \mathrm{E}+08$ \\
\hline 23 & 48 & 50.421 & $7.00 \mathrm{E}-04$ & $1.8 \mathrm{E}+09$ \\
\hline 23 & 50 & 49.161 & $4.28 \mathrm{E}-02$ & $3.9 \mathrm{E}+10$ \\
\hline 23 & 51 & 49.118 & $3.10 \mathrm{E}-01$ & $1.7 \mathrm{E}+11$ \\
\hline 23 & 55 & 49.012 & $2.17 \mathrm{E}+00$ & $1.2 \mathrm{E}+12$ \\
\hline 23 & 64 & 45.791 & $3.62 \mathrm{E}-01$ & $2.3 \mathrm{E}+11$ \\
\hline 24 & 30 & 57.695 & $1.40 \mathrm{E}-04$ & $9.4 \mathrm{E}+07$ \\
\hline 24 & 31 & 57.523 & $7.45 \mathrm{E}-04$ & $3.0 \mathrm{E}+08$ \\
\hline 24 & 32 & 57.217 & $2.67 \mathrm{E}-05$ & $7.8 \mathrm{E}+06$ \\
\hline 24 & 33 & 57.136 & $1.25 \mathrm{E}-03$ & $8.5 \mathrm{E}+08$ \\
\hline 24 & 34 & 56.965 & $3.96 \mathrm{E}-05$ & $1.6 \mathrm{E}+07$ \\
\hline 24 & 39 & 53.168 & $1.26 \mathrm{E}-01$ & $9.9 \mathrm{E}+10$ \\
\hline 24 & 44 & 52.796 & $6.52 \mathrm{E}-03$ & $5.2 \mathrm{E}+09$ \\
\hline 24 & 45 & 52.747 & $1.90 \mathrm{E}-02$ & $9.1 \mathrm{E}+09$ \\
\hline 24 & 50 & 50.941 & $2.99 \mathrm{E}-04$ & $2.6 \mathrm{E}+08$ \\
\hline 24 & 51 & 50.895 & $1.29 \mathrm{E}-04$ & $6.6 \mathrm{E}+07$ \\
\hline 24 & 54 & 50.798 & $1.11 \mathrm{E}-03$ & $4.1 \mathrm{E}+08$ \\
\hline 24 & 55 & 50.781 & $1.19 \mathrm{E}-02$ & $6.2 \mathrm{E}+09$ \\
\hline 24 & 56 & 50.758 & $4.47 \mathrm{E}-02$ & $1.7 \mathrm{E}+10$ \\
\hline 24 & 62 & 47.358 & $4.37 \mathrm{E}+00$ & $1.9 \mathrm{E}+12$ \\
\hline 24 & 63 & 47.333 & $3.20 \mathrm{E}-02$ & $1.4 \mathrm{E}+10$ \\
\hline 24 & 64 & 47.331 & $2.57 \mathrm{E}-01$ & $1.5 \mathrm{E}+11$ \\
\hline 25 & 30 & 57.979 & $5.87 \mathrm{E}-04$ & $3.9 \mathrm{E}+08$ \\
\hline 25 & 31 & 57.806 & $6.01 \mathrm{E}-04$ & $2.4 \mathrm{E}+08$ \\
\hline 25 & 32 & 57.497 & $7.40 \mathrm{E}-04$ & $2.1 \mathrm{E}+08$ \\
\hline 25 & 33 & 57.415 & $9.10 \mathrm{E}-04$ & $6.1 \mathrm{E}+08$ \\
\hline 25 & 34 & 57.243 & $3.03 E-03$ & $1.2 \mathrm{E}+09$ \\
\hline
\end{tabular}




\section{Continued}

\begin{tabular}{|c|c|c|c|c|}
\hline 25 & 39 & 53.41 & $8.47 \mathrm{E}-04$ & $6.6 \mathrm{E}+08$ \\
\hline 25 & 44 & 53.034 & $1.32 \mathrm{E}-01$ & $1.0 \mathrm{E}+11$ \\
\hline 25 & 45 & 52.984 & $1.81 \mathrm{E}-02$ & $8.6 \mathrm{E}+09$ \\
\hline 25 & 50 & 51.162 & $3.62 \mathrm{E}-03$ & $3.1 \mathrm{E}+09$ \\
\hline 25 & 51 & 51.116 & $3.71 \mathrm{E}-02$ & $1.9 \mathrm{E}+10$ \\
\hline 25 & 54 & 51.019 & 8.73E-02 & $3.2 \mathrm{E}+10$ \\
\hline 25 & 55 & 51.001 & $7.69 \mathrm{E}-04$ & $3.9 \mathrm{E}+08$ \\
\hline 25 & 56 & 50.979 & $1.93 \mathrm{E}-03$ & $7.1 \mathrm{E}+08$ \\
\hline 25 & 62 & 47.55 & $2.58 \mathrm{E}-03$ & $1.1 \mathrm{E}+09$ \\
\hline 25 & 63 & 47.525 & $4.33 \mathrm{E}+00$ & $1.8 \mathrm{E}+12$ \\
\hline 25 & 64 & 47.523 & $2.52 \mathrm{E}-01$ & $1.5 \mathrm{E}+11$ \\
\hline 26 & 31 & 57.994 & $1.17 \mathrm{E}-03$ & $4.6 \mathrm{E}+08$ \\
\hline 26 & 32 & 57.683 & $1.02 \mathrm{E}-03$ & $2.9 \mathrm{E}+08$ \\
\hline 26 & 34 & 57.427 & $1.60 \mathrm{E}-04$ & $6.5 \mathrm{E}+07$ \\
\hline 26 & 45 & 53.142 & $2.19 \mathrm{E}-01$ & $1.0 \mathrm{E}+11$ \\
\hline 26 & 51 & 51.263 & 8.73E-04 & $4.4 \mathrm{E}+08$ \\
\hline 26 & 52 & 51.239 & $3.37 \mathrm{E}-02$ & $9.5 E+09$ \\
\hline 26 & 54 & 51.165 & $1.07 \mathrm{E}-02$ & $3.9 \mathrm{E}+09$ \\
\hline 26 & 55 & 51.147 & $6.08 \mathrm{E}-04$ & $3.1 \mathrm{E}+08$ \\
\hline 26 & 56 & 51.125 & $4.35 \mathrm{E}-03$ & $1.6 \mathrm{E}+09$ \\
\hline 26 & 57 & 51.099 & $9.68 \mathrm{E}-03$ & $2.7 \mathrm{E}+09$ \\
\hline 26 & 62 & 47.677 & $2.91 \mathrm{E}-01$ & $1.2 \mathrm{E}+11$ \\
\hline 26 & 63 & 47.652 & $2.26 \mathrm{E}-01$ & $9.5 \mathrm{E}+10$ \\
\hline 26 & 64 & 47.65 & $1.46 \mathrm{E}-02$ & $8.6 \mathrm{E}+09$ \\
\hline 26 & 65 & 47.647 & $6.01 \mathrm{E}+00$ & $2.0 \mathrm{E}+12$ \\
\hline 27 & 30 & 60.1 & $1.11 \mathrm{E}-04$ & $6.8 \mathrm{E}+07$ \\
\hline 27 & 31 & 59.915 & $2.55 \mathrm{E}-05$ & $9.5 \mathrm{E}+06$ \\
\hline 27 & 33 & 59.495 & $1.22 \mathrm{E}-02$ & $7.7 \mathrm{E}+09$ \\
\hline 27 & 34 & 59.31 & $5.43 \mathrm{E}-04$ & $2.1 \mathrm{E}+08$ \\
\hline 27 & 37 & 58.198 & $2.52 \mathrm{E}-03$ & $5.0 \mathrm{E}+09$ \\
\hline 27 & 38 & 55.205 & $3.10 \mathrm{E}-02$ & $2.3 \mathrm{E}+10$ \\
\hline 27 & 39 & 54.803 & $1.67 \mathrm{E}-02$ & $1.2 \mathrm{E}+10$ \\
\hline 27 & 44 & 54.75 & $2.61 \mathrm{E}-03$ & $1.2 \mathrm{E}+09$ \\
\hline 27 & 48 & 54.264 & $5.73 \mathrm{E}-02$ & $1.3 \mathrm{E}+11$ \\
\hline 27 & 50 & 52.807 & $3.69 \mathrm{E}-03$ & $2.9 \mathrm{E}+09$ \\
\hline 27 & 51 & 52.758 & $1.55 \mathrm{E}-01$ & $7.4 \mathrm{E}+10$ \\
\hline 27 & 55 & 52.635 & $3.10 \mathrm{E}-01$ & $1.5 \mathrm{E}+11$ \\
\hline 27 & 64 & 48.939 & $2.49 \mathrm{E}+00$ & $1.4 \mathrm{E}+12$ \\
\hline 28 & 30 & 843.948 & $4.43 \mathrm{E}-01$ & $1.4 \mathrm{E}+09$ \\
\hline 28 & 31 & 808.723 & $3.61 \mathrm{E}-01$ & $7.4 \mathrm{E}+08$ \\
\hline 28 & 32 & 752.142 & $1.15 \mathrm{E}+00$ & $1.9 \mathrm{E}+09$ \\
\hline 28 & 33 & 738.356 & $2.29 \mathrm{E}-05$ & $9.3 \mathrm{E}+04$ \\
\hline 28 & 34 & 710.83 & $4.54 \mathrm{E}-01$ & $1.2 \mathrm{E}+09$ \\
\hline 28 & 39 & 375.88 & $1.01 \mathrm{E}-04$ & $1.6 \mathrm{E}+06$ \\
\hline 28 & 44 & 358.02 & $2.51 \mathrm{E}-03$ & $4.3 \mathrm{E}+07$ \\
\hline 28 & 45 & 355.77 & $5.01 \mathrm{E}-04$ & $5.3 \mathrm{E}+06$ \\
\hline 28 & 50 & 287.11 & $1.49 \mathrm{E}-06$ & $4.0 \mathrm{E}+04$ \\
\hline 28 & 51 & 285.67 & $1.72 \mathrm{E}-05$ & $2.8 \mathrm{E}+05$ \\
\hline 28 & 54 & 282.64 & $5.70 \mathrm{E}-05$ & $6.8 \mathrm{E}+05$ \\
\hline
\end{tabular}




\section{Continued}

\begin{tabular}{|c|c|c|c|c|}
\hline 28 & 55 & 282.1 & $4.59 \mathrm{E}-07$ & $7.7 \mathrm{E}+03$ \\
\hline 28 & 56 & 281.42 & $1.86 \mathrm{E}-06$ & $2.2 \mathrm{E}+04$ \\
\hline 28 & 62 & 201.29 & $8.49 \mathrm{E}-07$ & $2.0 \mathrm{E}+04$ \\
\hline 28 & 63 & 200.84 & $4.79 \mathrm{E}-05$ & $1.1 \mathrm{E}+06$ \\
\hline 28 & 64 & 200.81 & $3.42 \mathrm{E}-06$ & $1.1 \mathrm{E}+05$ \\
\hline 29 & 30 & 844.14 & $1.01 \mathrm{E}-07$ & $3.1 \mathrm{E}+02$ \\
\hline 29 & 31 & 808.9 & $4.04 \mathrm{E}-01$ & $8.2 E+08$ \\
\hline 29 & 33 & 738.51 & $4.99 \mathrm{E}-01$ & $2.0 \mathrm{E}+09$ \\
\hline 29 & 34 & 710.97 & $4.10 \mathrm{E}-01$ & $1.1 \mathrm{E}+09$ \\
\hline 29 & 37 & 578.51 & $2.03 \mathrm{E}-01$ & $4.0 \mathrm{E}+09$ \\
\hline 29 & 38 & 375.92 & $7.21 \mathrm{E}-04$ & $1.1 \mathrm{E}+07$ \\
\hline 29 & 39 & 358.06 & $3.27 \mathrm{E}-04$ & $5.7 \mathrm{E}+06$ \\
\hline 29 & 44 & 355.8 & $3.21 \mathrm{E}-04$ & $3.4 \mathrm{E}+06$ \\
\hline 29 & 48 & 336.23 & $1.76 \mathrm{E}-02$ & $1.0 \mathrm{E}+09$ \\
\hline 29 & 50 & 287.13 & $7.52 \mathrm{E}-06$ & $2.0 \mathrm{E}+05$ \\
\hline 29 & 51 & 285.7 & $1.30 \mathrm{E}-06$ & $2.1 \mathrm{E}+04$ \\
\hline 29 & 55 & 282.13 & $3.48 \mathrm{E}-05$ & $5.8 \mathrm{E}+05$ \\
\hline 29 & 64 & 200.82 & $2.99 \mathrm{E}-05$ & $9.9 \mathrm{E}+05$ \\
\hline 30 & 35 & 4473.3 & $1.85 \mathrm{E}-04$ & $6.2 \mathrm{E}+04$ \\
\hline 30 & 36 & 4470.5 & $2.16 \mathrm{E}-04$ & $2.4 \mathrm{E}+04$ \\
\hline 30 & 38 & 684.86 & $2.01 \mathrm{E}-01$ & $2.9 \mathrm{E}+09$ \\
\hline 30 & 40 & 663.15 & $4.48 \mathrm{E}-01$ & $2.3 E+09$ \\
\hline 30 & 43 & 626.03 & $3.28 \mathrm{E}-01$ & $1.1 \mathrm{E}+09$ \\
\hline 30 & 47 & 583.98 & $5.93 \mathrm{E}-02$ & $2.3 \mathrm{E}+08$ \\
\hline 30 & 49 & 540.3 & $2.44 \mathrm{E}-02$ & $1.9 \mathrm{E}+08$ \\
\hline 30 & 58 & 332.57 & $1.99 \mathrm{E}-04$ & $2.4 \mathrm{E}+06$ \\
\hline 30 & 60 & 328.41 & $1.44 \mathrm{E}-04$ & $1.8 \mathrm{E}+06$ \\
\hline 30 & 61 & 315.36 & $4.49 \mathrm{E}-04$ & $1.0 \mathrm{E}+07$ \\
\hline 31 & 36 & 5811.3 & $7.03 \mathrm{E}-05$ & $4.6 \mathrm{E}+03$ \\
\hline 31 & 40 & 686.65 & $5.74 \mathrm{E}-02$ & $2.7 \mathrm{E}+08$ \\
\hline 31 & 42 & 658.95 & $1.20 \mathrm{E}+00$ & $2.6 \mathrm{E}+09$ \\
\hline 31 & 43 & 646.93 & $1.33 \mathrm{E}-01$ & $4.2 \mathrm{E}+08$ \\
\hline 31 & 46 & 606.97 & $3.24 \mathrm{E}-02$ & $8.4 \mathrm{E}+07$ \\
\hline 31 & 47 & 602.13 & $3.24 \mathrm{E}-01$ & $1.2 \mathrm{E}+09$ \\
\hline 31 & 49 & 555.8 & $2.56 \mathrm{E}-02$ & $1.8 \mathrm{E}+08$ \\
\hline 31 & 58 & 338.38 & $2.59 \mathrm{E}-03$ & $3.0 \mathrm{E}+07$ \\
\hline 31 & 59 & 336.08 & $2.99 \mathrm{E}-06$ & $2.5 E+04$ \\
\hline 31 & 60 & 334.07 & $3.52 \mathrm{E}-04$ & $4.2 \mathrm{E}+06$ \\
\hline 31 & 61 & 320.58 & $2.52 \mathrm{E}-04$ & $5.5 \mathrm{E}+06$ \\
\hline 32 & 41 & 706.12 & $1.76 \mathrm{E}+00$ & $2.6 \mathrm{E}+09$ \\
\hline 32 & 42 & 701.98 & $1.07 \mathrm{E}-01$ & $2.1 \mathrm{E}+08$ \\
\hline 32 & 43 & 688.35 & $5.42 \mathrm{E}-02$ & $1.5 \mathrm{E}+08$ \\
\hline 32 & 46 & 643.29 & $3.83 \mathrm{E}-01$ & $8.8 \mathrm{E}+08$ \\
\hline 32 & 47 & 637.85 & $1.04 \mathrm{E}-02$ & $3.4 \mathrm{E}+07$ \\
\hline 32 & 58 & 349.37 & $3.70 \mathrm{E}-04$ & $4.0 \mathrm{E}+06$ \\
\hline 32 & 59 & 346.93 & $2.32 \mathrm{E}-03$ & $1.8 \mathrm{E}+07$ \\
\hline 32 & 60 & 344.79 & $7.71 \mathrm{E}-04$ & 8.7E+06 \\
\hline 33 & 35 & 18486.8 & $4.93 \mathrm{E}-08$ & $9.6 \mathrm{E}-01$ \\
\hline 33 & 36 & 18438.6 & $3.67 \mathrm{E}-05$ & $2.4 \mathrm{E}+02$ \\
\hline
\end{tabular}




\section{Continued}

\begin{tabular}{|c|c|c|c|c|}
\hline 33 & 38 & 774.775 & $2.41 \mathrm{E}-06$ & $2.7 \mathrm{E}+04$ \\
\hline 33 & 40 & 747.104 & $9.44 \mathrm{E}-03$ & $3.8 \mathrm{E}+07$ \\
\hline 33 & 43 & 700.319 & $1.05 \mathrm{E}-01$ & $2.8 \mathrm{E}+08$ \\
\hline 33 & 47 & 648.112 & $6.81 \mathrm{E}-01$ & $2.2 \mathrm{E}+09$ \\
\hline 33 & 49 & 594.755 & $2.77 \mathrm{E}-01$ & $1.7 \mathrm{E}+09$ \\
\hline 33 & 58 & 352.43 & $8.89 \mathrm{E}-04$ & $9.5 \mathrm{E}+06$ \\
\hline 33 & 60 & 347.763 & $3.61 \mathrm{E}-03$ & $4.0 \mathrm{E}+07$ \\
\hline 33 & 61 & 333.169 & $1.77 \mathrm{E}-03$ & $3.5 \mathrm{E}+07$ \\
\hline 34 & 40 & 777.569 & 7.67E-02 & $2.8 \mathrm{E}+08$ \\
\hline 34 & 42 & 742.239 & $1.30 \mathrm{E}-01$ & $2.3 \mathrm{E}+08$ \\
\hline 34 & 43 & 727.019 & $3.92 \mathrm{E}-01$ & $9.9 \mathrm{E}+08$ \\
\hline 34 & 46 & 676.94 & $1.03 \mathrm{E}+00$ & $2.1 \mathrm{E}+09$ \\
\hline 34 & 47 & 670.915 & $1.25 \mathrm{E}-02$ & $3.7 \mathrm{E}+07$ \\
\hline 34 & 49 & 613.902 & $7.67 \mathrm{E}-04$ & $4.5 \mathrm{E}+06$ \\
\hline 34 & 58 & 359.066 & $2.74 \mathrm{E}-04$ & $2.8 \mathrm{E}+06$ \\
\hline 34 & 59 & 356.482 & $2.58 \mathrm{E}-03$ & $1.9 \mathrm{E}+07$ \\
\hline 34 & 60 & 354.223 & $7.83 \mathrm{E}-03$ & $8.3 \mathrm{E}+07$ \\
\hline 34 & 61 & 339.093 & 8.95E-04 & $1.7 \mathrm{E}+07$ \\
\hline 35 & 39 & 798.761 & $1.62 \mathrm{E}-01$ & $5.6 \mathrm{E}+08$ \\
\hline 35 & 44 & 722.196 & $3.34 \mathrm{E}-01$ & $1.4 \mathrm{E}+09$ \\
\hline 35 & 50 & 482.038 & $7.35 \mathrm{E}-05$ & $7.0 \mathrm{E}+05$ \\
\hline 36 & 34 & 3122.43 & $1.75 \mathrm{E}-03$ & $1.2 \mathrm{E}+06$ \\
\hline 36 & 37 & 798.851 & $3.03 \mathrm{E}-01$ & $1.1 \mathrm{E}+09$ \\
\hline 36 & 38 & 722.269 & $1.77 \mathrm{E}-01$ & $7.6 \mathrm{E}+08$ \\
\hline 36 & 39 & 713.152 & 8.67E-01 & $2.3 \mathrm{E}+09$ \\
\hline 36 & 44 & 638.627 & $1.84 \mathrm{E}-01$ & $3.0 \mathrm{E}+09$ \\
\hline 36 & 48 & 482.071 & $3.61 \mathrm{E}-05$ & $3.5 \mathrm{E}+05$ \\
\hline 36 & 50 & 478.031 & $1.41 \mathrm{E}-04$ & $8.2 E+05$ \\
\hline 36 & 51 & 468.122 & $1.04 \mathrm{E}-05$ & $6.3 \mathrm{E}+04$ \\
\hline 36 & 55 & 280.012 & $4.05 \mathrm{E}-07$ & $6.9 \mathrm{E}+03$ \\
\hline 37 & 40 & 1037.34 & $1.01 \mathrm{E}-02$ & $2.1 \mathrm{E}+07$ \\
\hline 37 & 49 & 765.189 & $2.75 \mathrm{E}-01$ & $1.0 \mathrm{E}+09$ \\
\hline 37 & 61 & 380.665 & $1.15 \mathrm{E}-02$ & $1.8 \mathrm{E}+08$ \\
\hline 38 & 39 & 65210.5 & $3.80 \mathrm{E}-08$ & $2.0 \mathrm{E}-02$ \\
\hline 38 & 44 & 6753.94 & $1.09 \mathrm{E}-04$ & $5.3 \mathrm{E}+03$ \\
\hline 38 & 50 & 1193.43 & $1.11 \mathrm{E}-01$ & $1.7 \mathrm{E}+08$ \\
\hline 38 & 40 & 30798.8 & $2.92 \mathrm{E}-06$ & $6.8 \mathrm{E}+00$ \\
\hline 38 & 49 & 2664.3 & $6.64 \mathrm{E}-05$ & $2.1 \mathrm{E}+04$ \\
\hline 38 & 61 & 589.814 & $1.90 \mathrm{E}-01$ & $1.2 \mathrm{E}+09$ \\
\hline 39 & 43 & 8204.16 & $1.49 \mathrm{E}-06$ & $2.9 \mathrm{E}+01$ \\
\hline 39 & 49 & 4121.91 & $5.96 \mathrm{E}-05$ & $7.8 \mathrm{E}+03$ \\
\hline 39 & 58 & 652.991 & $7.13 \mathrm{E}-01$ & $2.2 \mathrm{E}+09$ \\
\hline 39 & 60 & 637.15 & $1.74 \mathrm{E}-01$ & $5.7 \mathrm{E}+08$ \\
\hline 39 & 61 & 639.909 & $9.38 \mathrm{E}-02$ & $5.1 \mathrm{E}+08$ \\
\hline 40 & 39 & 9974.27 & $3.03 \mathrm{E}-05$ & $6.8 \mathrm{E}+02$ \\
\hline 40 & 44 & 8477.57 & $6.15 \mathrm{E}-05$ & $1.1 \mathrm{E}+03$ \\
\hline 40 & 48 & 3551.25 & $7.96 \mathrm{E}-05$ & $4.2 \mathrm{E}+04$ \\
\hline 40 & 50 & 1265.64 & $9.98 \mathrm{E}-02$ & $1.4 \mathrm{E}+08$ \\
\hline 40 & 51 & 1238.16 & $1.99 \mathrm{E}-01$ & $1.7 \mathrm{E}+08$ \\
\hline
\end{tabular}




\section{Continued}

\begin{tabular}{|c|c|c|c|c|}
\hline 40 & 55 & 1173.81 & $1.95 \mathrm{E}-02$ & $1.9 \mathrm{E}+07$ \\
\hline 40 & 64 & 437.254 & $3.01 \mathrm{E}-03$ & $2.1 \mathrm{E}+07$ \\
\hline 41 & 52 & 1308.53 & $2.53 \mathrm{E}-02$ & $1.1 \mathrm{E}+07$ \\
\hline 41 & 53 & 1300.2 & $7.98 \mathrm{E}-01$ & $2.9 \mathrm{E}+08$ \\
\hline 41 & 54 & 1262.2 & $6.00 \mathrm{E}-03$ & $3.6 \mathrm{E}+06$ \\
\hline 41 & 56 & 1238.1 & $1.49 \mathrm{E}-03$ & $9.3 \mathrm{E}+05$ \\
\hline 41 & 57 & 1223.4 & $8.85 E-02$ & $4.4 \mathrm{E}+07$ \\
\hline 41 & 62 & 449.98 & $1.49 \mathrm{E}-06$ & $7.0 \mathrm{E}+03$ \\
\hline 41 & 63 & 447.76 & $1.31 \mathrm{E}-05$ & $6.2 \mathrm{E}+04$ \\
\hline 41 & 65 & 447.36 & $9.91 \mathrm{E}-05$ & $3.7 \mathrm{E}+05$ \\
\hline 42 & 45 & 17623 & $4.10 \mathrm{E}-08$ & $1.8 \mathrm{E}-01$ \\
\hline 42 & 51 & 1339.7 & $9.75 \mathrm{E}-04$ & $7.2 \mathrm{E}+05$ \\
\hline 42 & 52 & 1323 & $6.12 \mathrm{E}-01$ & $2.6 \mathrm{E}+08$ \\
\hline 42 & 54 & 1275.6 & $5.97 \mathrm{E}-03$ & $3.5 \mathrm{E}+06$ \\
\hline 42 & 55 & 1264.7 & $3.68 \mathrm{E}-03$ & $3.1 \mathrm{E}+06$ \\
\hline 42 & 56 & 1251 & $7.53 \mathrm{E}-02$ & $4.6 \mathrm{E}+07$ \\
\hline 42 & 57 & 1236 & $9.71 \mathrm{E}-04$ & $4.7 \mathrm{E}+05$ \\
\hline 42 & 62 & 451.68 & $9.16 \mathrm{E}-06$ & $4.3 \mathrm{E}+04$ \\
\hline 42 & 63 & 449.44 & $6.08 \mathrm{E}-05$ & $2.9 \mathrm{E}+05$ \\
\hline 42 & 64 & 449.28 & $1.64 \mathrm{E}-05$ & $1.1 \mathrm{E}+05$ \\
\hline 42 & 65 & 449.04 & $5.06 \mathrm{E}-04$ & $1.9 \mathrm{E}+06$ \\
\hline 43 & 44 & 92272 & $2.16 \mathrm{E}-05$ & $5.7 \mathrm{E}+00$ \\
\hline 43 & 45 & 35041 & $4.13 \mathrm{E}-05$ & $4.5 \mathrm{E}+01$ \\
\hline 43 & 50 & 1427.2 & $1.12 \mathrm{E}-02$ & $1.2 \mathrm{E}+07$ \\
\hline 43 & 51 & 1392.3 & $1.12 \mathrm{E}-01$ & $7.7 \mathrm{E}+07$ \\
\hline 43 & 54 & 1323.2 & $3.54 \mathrm{E}-01$ & $1.9 \mathrm{E}+08$ \\
\hline 43 & 55 & 1311.5 & $1.09 \mathrm{E}-02$ & $8.5 \mathrm{E}+06$ \\
\hline 43 & 56 & 1296.8 & $2.77 \mathrm{E}-04$ & $1.6 \mathrm{E}+05$ \\
\hline 43 & 62 & 457.51 & $6.71 \mathrm{E}-05$ & $3.1 \mathrm{E}+05$ \\
\hline 43 & 63 & 455.21 & $7.31 \mathrm{E}-03$ & $3.4 \mathrm{E}+07$ \\
\hline 43 & 64 & 455.05 & $2.86 \mathrm{E}-04$ & $1.8 \mathrm{E}+06$ \\
\hline 44 & 49 & 4446.3 & $2.93 \mathrm{E}-05$ & $3.3 \mathrm{E}+03$ \\
\hline 44 & 58 & 714.96 & $2.72 \mathrm{E}-01$ & $7.1 \mathrm{E}+08$ \\
\hline 44 & 60 & 696.01 & $6.21 \mathrm{E}-01$ & $1.7 \mathrm{E}+09$ \\
\hline 44 & 61 & 647.24 & $1.72 \mathrm{E}-02$ & $9.1 \mathrm{E}+07$ \\
\hline 45 & 46 & 13658 & $1.20 \mathrm{E}-04$ & $6.1 \mathrm{E}+02$ \\
\hline 45 & 58 & 724.12 & $3.92 \mathrm{E}-02$ & $1.0 \mathrm{E}+08$ \\
\hline 45 & 59 & 713.69 & $1.35 \mathrm{E}+00$ & $2.5 \mathrm{E}+09$ \\
\hline 45 & 60 & 704.69 & $2.02 \mathrm{E}-01$ & $5.4 \mathrm{E}+08$ \\
\hline 46 & 51 & 1622.1 & $8.00 \mathrm{E}-03$ & $4.1 \mathrm{E}+06$ \\
\hline 46 & 52 & 1597.7 & $6.37 \mathrm{E}-04$ & $1.9 \mathrm{E}+05$ \\
\hline 46 & 54 & 1529.1 & $1.07 \mathrm{E}-01$ & $4.4 \mathrm{E}+07$ \\
\hline 46 & 55 & 1513.4 & $1.20 \mathrm{E}-03$ & $7.0 \mathrm{E}+05$ \\
\hline 46 & 56 & 1493.9 & $2.06 \mathrm{E}-03$ & $8.8 \mathrm{E}+05$ \\
\hline 46 & 57 & 1472.5 & $4.98 \mathrm{E}-01$ & $1.7 \mathrm{E}+08$ \\
\hline 46 & 62 & 479.85 & $1.63 \mathrm{E}-04$ & $6.8 \mathrm{E}+05$ \\
\hline 46 & 63 & 477.32 & $8.71 \mathrm{E}-04$ & $3.6 \mathrm{E}+06$ \\
\hline 46 & 64 & 477.14 & $4.71 \mathrm{E}-05$ & $2.8 \mathrm{E}+05$ \\
\hline 46 & 65 & 476.87 & $1.81 \mathrm{E}-03$ & $5.9 \mathrm{E}+06$ \\
\hline
\end{tabular}




\section{Continued}

\begin{tabular}{|c|c|c|c|c|}
\hline 47 & 39 & 4221 & $3.61 \mathrm{E}-04$ & $2.7 \mathrm{E}+04$ \\
\hline 47 & 44 & 9598.5 & $3.62 \mathrm{E}-06$ & $5.2 \mathrm{E}+01$ \\
\hline 47 & 45 & 11563 & $3.40 \mathrm{E}-05$ & $3.4 \mathrm{E}+02$ \\
\hline 47 & 50 & 1707.4 & $1.54 \mathrm{E}-03$ & $1.2 \mathrm{E}+06$ \\
\hline 47 & 51 & 1657.8 & $6.76 \mathrm{E}-05$ & $3.3 \mathrm{E}+04$ \\
\hline 47 & 54 & 1560.8 & $4.90 \mathrm{E}-03$ & $1.9 \mathrm{E}+06$ \\
\hline 47 & 55 & 1544.4 & $5.70 \mathrm{E}-02$ & $3.2 \mathrm{E}+07$ \\
\hline 47 & 56 & 1524.1 & $3.64 \mathrm{E}-01$ & $1.5 \mathrm{E}+08$ \\
\hline 47 & 62 & 482.923 & $3.98 \mathrm{E}-03$ & $1.6 \mathrm{E}+07$ \\
\hline 47 & 63 & 480.362 & $3.20 \mathrm{E}-04$ & $1.3 \mathrm{E}+06$ \\
\hline 47 & 64 & 480.178 & $1.32 \mathrm{E}-03$ & $7.6 \mathrm{E}+06$ \\
\hline 48 & 49 & 16320.5 & $2.06 \mathrm{E}-04$ & $1.7 \mathrm{E}+03$ \\
\hline 48 & 61 & 723.909 & $3.05 \mathrm{E}-01$ & $1.3 \mathrm{E}+09$ \\
\hline 49 & 50 & 2235.87 & $2.47 \mathrm{E}-03$ & $1.1 \mathrm{E}+06$ \\
\hline 49 & 51 & 2151.54 & $2.28 \mathrm{E}-02$ & $6.6 \mathrm{E}+06$ \\
\hline 49 & 55 & 1964.39 & $1.66 \mathrm{E}-01$ & $5.7 \mathrm{E}+07$ \\
\hline 49 & 64 & 514.367 & $7.67 \mathrm{E}-03$ & $3.9 \mathrm{E}+07$ \\
\hline 50 & 58 & 1410.78 & $3.11 \mathrm{E}-05$ & $2.1 \mathrm{E}+04$ \\
\hline 50 & 60 & 1338.86 & $5.47 \mathrm{E}-05$ & $4.1 \mathrm{E}+04$ \\
\hline 50 & 61 & 1145.65 & $2.96 \mathrm{E}-04$ & $5.0 \mathrm{E}+05$ \\
\hline 51 & 58 & 1446.56 & $1.36 \mathrm{E}-04$ & $8.7 \mathrm{E}+04$ \\
\hline 51 & 59 & 1405.52 & $3.13 \mathrm{E}-05$ & $1.5 \mathrm{E}+04$ \\
\hline 51 & 60 & 1371.04 & $6.81 \mathrm{E}-04$ & $4.8 \mathrm{E}+05$ \\
\hline 51 & 61 & 1169.14 & $1.31 \mathrm{E}-03$ & $2.1 \mathrm{E}+06$ \\
\hline 52 & 59 & 1424.41 & $5.92 \mathrm{E}-05$ & $2.8 \mathrm{E}+04$ \\
\hline 54 & 58 & 1529.54 & $1.96 \mathrm{E}-05$ & $1.1 \mathrm{E}+04$ \\
\hline 54 & 59 & 1483.74 & $4.47 \mathrm{E}-04$ & $1.9 \mathrm{E}+05$ \\
\hline 54 & 60 & 1445.37 & $1.33 \mathrm{E}-03$ & $8.5 E+05$ \\
\hline 55 & 58 & 1545.55 & $3.17 \mathrm{E}-04$ & $1.8 \mathrm{E}+05$ \\
\hline 55 & 59 & 1498.8 & $1.04 \mathrm{E}-05$ & $4.4 \mathrm{E}+03$ \\
\hline 55 & 60 & 1459.66 & $5.74 \mathrm{E}-05$ & $3.6 \mathrm{E}+04$ \\
\hline 55 & 61 & 1232.96 & $1.41 \mathrm{E}-03$ & $2.1 \mathrm{E}+06$ \\
\hline 56 & 58 & 1566.42 & $5.28 \mathrm{E}-04$ & $2.9 \mathrm{E}+05$ \\
\hline 56 & 59 & 1518.41 & $6.08 \mathrm{E}-06$ & $2.5 E+03$ \\
\hline 56 & 60 & 1478.25 & $5.41 \mathrm{E}-04$ & $3.3 \mathrm{E}+05$ \\
\hline 57 & 59 & 1541.22 & $3.46 \mathrm{E}-04$ & $1.4 \mathrm{E}+05$ \\
\hline 58 & 62 & 1288.3 & $4.06 \mathrm{E}-01$ & $2.3 \mathrm{E}+08$ \\
\hline 58 & 63 & 1270.23 & $9.57 \mathrm{E}-02$ & $5.7 \mathrm{E}+07$ \\
\hline 58 & 64 & 1268.94 & $9.31 \mathrm{E}-03$ & 7.7E+06 \\
\hline 59 & 62 & 1322.69 & $3.08 \mathrm{E}-02$ & $1.7 \mathrm{E}+07$ \\
\hline 59 & 63 & 1303.65 & $2.44 \mathrm{E}-02$ & $1.4 \mathrm{E}+07$ \\
\hline 59 & 64 & 1302.3 & $1.58 \mathrm{E}-03$ & $1.2 \mathrm{E}+06$ \\
\hline 59 & 65 & 1300.26 & $6.49 \mathrm{E}-01$ & $2.8 \mathrm{E}+08$ \\
\hline 60 & 62 & 1354.75 & $6.81 \mathrm{E}-02$ & $3.5 \mathrm{E}+07$ \\
\hline 60 & 63 & 1334.79 & $3.75 \mathrm{E}-01$ & $2.0 \mathrm{E}+08$ \\
\hline 60 & 64 & 1333.36 & $4.55 \mathrm{E}-02$ & $3.4 \mathrm{E}+07$ \\
\hline 61 & 64 & 1602.51 & $2.39 \mathrm{E}-01$ & $1.2 \mathrm{E}+08$ \\
\hline
\end{tabular}


Table 6. The allowed electric dipole transitions for $\mathrm{Cu} \mathrm{XX}$.

\begin{tabular}{|c|c|c|c|c|}
\hline i & j & $\lambda(\AA)$ & gf & Aji \\
\hline 1 & 3 & 12.828 & $1.00 \mathrm{E}+00$ & $0.00 \mathrm{E}+00$ \\
\hline 1 & 5 & 12.571 & $1.00 \mathrm{E}+00$ & $0.00 \mathrm{E}+00$ \\
\hline 1 & 17 & 11.736 & $1.00 \mathrm{E}+00$ & $0.00 \mathrm{E}+00$ \\
\hline 1 & 23 & 11.593 & $1.00 \mathrm{E}+00$ & $0.00 \mathrm{E}+00$ \\
\hline 1 & 27 & 11.38 & $1.00 \mathrm{E}+00$ & $0.00 \mathrm{E}+00$ \\
\hline 1 & 29 & 9.521 & $1.00 \mathrm{E}+00$ & $0.00 \mathrm{E}+00$ \\
\hline 1 & 36 & 9.373 & $1.00 \mathrm{E}+00$ & $0.00 \mathrm{E}+00$ \\
\hline 1 & 39 & 9.268 & $1.00 \mathrm{E}+00$ & $0.00 \mathrm{E}+00$ \\
\hline 1 & 48 & 9.236 & $1.00 \mathrm{E}+00$ & $0.00 \mathrm{E}+00$ \\
\hline 1 & 61 & 9.104 & $1.00 \mathrm{E}+00$ & $0.00 \mathrm{E}+00$ \\
\hline 2 & 6 & 340.94 & $1.00 \mathrm{E}+00$ & $0.00 \mathrm{E}+00$ \\
\hline 2 & 7 & 311.332 & $1.00 \mathrm{E}+00$ & $0.00 \mathrm{E}+00$ \\
\hline 2 & 8 & 287.005 & $1.00 \mathrm{E}+00$ & $0.00 \mathrm{E}+00$ \\
\hline 2 & 9 & 280.976 & $1.00 \mathrm{E}+00$ & $0.00 \mathrm{E}+00$ \\
\hline 2 & 10 & 265.351 & $1.00 \mathrm{E}+00$ & $0.00 \mathrm{E}+00$ \\
\hline 2 & 12 & 207.397 & $1.00 \mathrm{E}+00$ & $0.00 \mathrm{E}+00$ \\
\hline 2 & 13 & 190.629 & $1.00 \mathrm{E}+00$ & $0.00 \mathrm{E}+00$ \\
\hline 2 & 14 & 189.246 & $1.00 \mathrm{E}+00$ & $0.00 \mathrm{E}+00$ \\
\hline 2 & 30 & 35.093 & $1.00 \mathrm{E}+00$ & $0.00 \mathrm{E}+00$ \\
\hline 2 & 31 & 35.029 & $1.00 \mathrm{E}+00$ & $0.00 \mathrm{E}+00$ \\
\hline 2 & 32 & 34.888 & $1.00 \mathrm{E}+00$ & $0.00 \mathrm{E}+00$ \\
\hline 2 & 33 & 34.859 & $1.00 \mathrm{E}+00$ & $0.00 \mathrm{E}+00$ \\
\hline 2 & 34 & 34.789 & $1.00 \mathrm{E}+00$ & $0.00 \mathrm{E}+00$ \\
\hline 2 & 43 & 33.09 & $1.00 \mathrm{E}+00$ & $0.00 \mathrm{E}+00$ \\
\hline 2 & 46 & 32.918 & $1.00 \mathrm{E}+00$ & $0.00 \mathrm{E}+00$ \\
\hline 2 & 47 & 32.898 & $1.00 \mathrm{E}+00$ & $0.00 \mathrm{E}+00$ \\
\hline 2 & 50 & 32.314 & $1.00 \mathrm{E}+00$ & $0.00 \mathrm{E}+00$ \\
\hline 2 & 51 & 32.293 & $1.00 \mathrm{E}+00$ & $0.00 \mathrm{E}+00$ \\
\hline 2 & 54 & 32.251 & $1.00 \mathrm{E}+00$ & $0.00 \mathrm{E}+00$ \\
\hline 2 & 55 & 32.244 & $1.00 \mathrm{E}+00$ & $0.00 \mathrm{E}+00$ \\
\hline 2 & 56 & 32.235 & $1.00 \mathrm{E}+00$ & $0.00 \mathrm{E}+00$ \\
\hline 2 & 62 & 30.595 & $1.00 \mathrm{E}+00$ & $0.00 \mathrm{E}+00$ \\
\hline 2 & 63 & 30.583 & $1.00 \mathrm{E}+00$ & $0.00 \mathrm{E}+00$ \\
\hline 2 & 64 & 30.582 & $1.00 \mathrm{E}+00$ & $0.00 \mathrm{E}+00$ \\
\hline 3 & 6 & 363.787 & $1.00 \mathrm{E}+00$ & $0.00 \mathrm{E}+00$ \\
\hline 3 & 7 & 330.274 & $1.00 \mathrm{E}+00$ & $0.00 \mathrm{E}+00$ \\
\hline 3 & 9 & 296.313 & $1.00 \mathrm{E}+00$ & $0.00 \mathrm{E}+00$ \\
\hline 3 & 10 & 278.988 & $1.00 \mathrm{E}+00$ & $0.00 \mathrm{E}+00$ \\
\hline 3 & 11 & 235.456 & $1.00 \mathrm{E}+00$ & $0.00 \mathrm{E}+00$ \\
\hline 3 & 12 & 215.636 & $1.00 \mathrm{E}+00$ & \\
\hline 3 & 13 & 197.567 & $1.00 \mathrm{E}+00$ & $0.00 \mathrm{E}+00$ \\
\hline 3 & 14 & 196.082 & $1.00 \mathrm{E}+00$ & $0.00 \mathrm{E}+00$ \\
\hline 3 & 15 & 163.58 & $1.00 \mathrm{E}+00$ & $0.00 \mathrm{E}+00$ \\
\hline 3 & 30 & 35.321 & $1.00 \mathrm{E}+00$ & $0.00 \mathrm{E}+00$ \\
\hline 3 & 31 & 35.257 & $1.00 \mathrm{E}+00$ & $0.00 \mathrm{E}+00$ \\
\hline 3 & 33 & 35.084 & $1.00 \mathrm{E}+00$ & $0.00 \mathrm{E}+00$ \\
\hline 3 & 34 & 35.013 & $1.00 \mathrm{E}+00$ & $0.00 \mathrm{E}+00$ \\
\hline
\end{tabular}




\section{Continued}

\begin{tabular}{|c|c|c|c|c|}
\hline 3 & 55 & 32.437 & $1.14 \mathrm{E}-04$ & $1.44 \mathrm{E}+08$ \\
\hline 3 & 63 & 30.756 & $1.11 \mathrm{E}-04$ & $1.57 \mathrm{E}+08$ \\
\hline 4 & 6 & 790.026 & $1.43 \mathrm{E}-03$ & $5.10 \mathrm{E}+06$ \\
\hline 4 & 9 & 528.615 & $1.49 \mathrm{E}-04$ & $1.19 \mathrm{E}+06$ \\
\hline 4 & 12 & 317.02 & $1.07 \mathrm{E}-01$ & $2.38 \mathrm{E}+09$ \\
\hline 4 & 13 & 279.447 & $2.12 \mathrm{E}-01$ & $6.04 \mathrm{E}+09$ \\
\hline 4 & 30 & 37.273 & $6.08 \mathrm{E}-04$ & $9.74 \mathrm{E}+08$ \\
\hline 4 & 33 & 37.01 & $6.78 \mathrm{E}-08$ & $1.10 \mathrm{E}+05$ \\
\hline 4 & 43 & 35.022 & 8.99E-02 & $1.63 E+11$ \\
\hline 4 & 46 & 34.83 & $1.71 \mathrm{E}-01$ & $3.14 \mathrm{E}+11$ \\
\hline 4 & 50 & 34.154 & $1.99 \mathrm{E}-04$ & $3.80 \mathrm{E}+08$ \\
\hline 5 & 6 & 865.536 & $1.82 \mathrm{E}-03$ & $5.41 \mathrm{E}+06$ \\
\hline 5 & 7 & 697.211 & $6.04 \mathrm{E}-06$ & $1.66 \mathrm{E}+04$ \\
\hline 5 & 9 & 561.385 & $1.01 \mathrm{E}-04$ & $7.15 E+05$ \\
\hline 5 & 10 & 502.29 & $2.41 \mathrm{E}-03$ & $1.28 \mathrm{E}+07$ \\
\hline 5 & 11 & 376.851 & $1.96 \mathrm{E}-02$ & $9.22 \mathrm{E}+08$ \\
\hline 5 & 12 & 328.521 & $1.83 \mathrm{E}-01$ & $3.78 \mathrm{E}+09$ \\
\hline 5 & 13 & 288.344 & $1.14 \mathrm{E}-01$ & $3.03 E+09$ \\
\hline 5 & 14 & 285.193 & $5.50 \mathrm{E}-01$ & $9.01 E+09$ \\
\hline 5 & 15 & 221.253 & $1.11 \mathrm{E}-01$ & $1.51 \mathrm{E}+10$ \\
\hline 5 & 30 & 37.427 & $7.53 \mathrm{E}-04$ & $1.20 \mathrm{E}+09$ \\
\hline 5 & 31 & 37.356 & $8.83 \mathrm{E}-04$ & $8.45 E+08$ \\
\hline 5 & 33 & 37.162 & $8.20 \mathrm{E}-04$ & $1.32 \mathrm{E}+09$ \\
\hline 5 & 34 & 37.082 & $2.48 \mathrm{E}-03$ & $2.41 E+09$ \\
\hline 5 & 37 & 36.616 & $1.86 \mathrm{E}-03$ & $9.24 \mathrm{E}+09$ \\
\hline 5 & 43 & 35.158 & $1.73 \mathrm{E}-01$ & $3.12 \mathrm{E}+11$ \\
\hline 5 & 46 & 34.964 & $9.12 \mathrm{E}-02$ & $1.66 \mathrm{E}+11$ \\
\hline 5 & 47 & 34.941 & $4.39 \mathrm{E}-01$ & $4.79 \mathrm{E}+11$ \\
\hline 5 & 49 & 34.765 & $7.66 \mathrm{E}-02$ & $4.22 \mathrm{E}+11$ \\
\hline 5 & 50 & 34.283 & 8.93E-05 & $1.69 \mathrm{E}+08$ \\
\hline 5 & 51 & 34.26 & $3.80 \mathrm{E}-04$ & $4.33 \mathrm{E}+08$ \\
\hline 5 & 55 & 34.205 & $5.15 \mathrm{E}-05$ & $5.88 \mathrm{E}+07$ \\
\hline 5 & 63 & 32.341 & $2.90 \mathrm{E}-09$ & $3.71 \mathrm{E}+03$ \\
\hline 6 & 16 & 227.802 & $1.20 \mathrm{E}-01$ & $5.15 E+09$ \\
\hline 6 & 17 & 222.126 & $2.80 \mathrm{E}-01$ & $1.26 \mathrm{E}+10$ \\
\hline 6 & 19 & 212.714 & $2.12 \mathrm{E}-01$ & $1.04 \mathrm{E}+10$ \\
\hline 6 & 21 & 203.21 & $3.14 \mathrm{E}-02$ & $1.69 \mathrm{E}+09$ \\
\hline 6 & 23 & 180.122 & $3.76 \mathrm{E}-03$ & $2.57 \mathrm{E}+08$ \\
\hline 6 & 24 & 156.119 & $8.39 \mathrm{E}-04$ & $7.66 \mathrm{E}+07$ \\
\hline 6 & 25 & 153.758 & $1.73 \mathrm{E}-03$ & $1.62 \mathrm{E}+08$ \\
\hline 6 & 27 & 139.507 & $2.20 \mathrm{E}-07$ & $2.51 \mathrm{E}+04$ \\
\hline 6 & 28 & 41.107 & $1.65 \mathrm{E}-01$ & $2.17 \mathrm{E}+11$ \\
\hline 6 & 29 & 41.107 & $1.62 \mathrm{E}-03$ & $2.13 E+09$ \\
\hline 6 & 35 & 38.48 & $2.02 \mathrm{E}-03$ & $3.03 E+09$ \\
\hline 6 & 36 & 38.479 & $3.27 \mathrm{E}-03$ & $4.91 \mathrm{E}+09$ \\
\hline 6 & 38 & 36.84 & $2.19 \mathrm{E}-01$ & $3.59 \mathrm{E}+11$ \\
\hline 6 & 39 & 36.776 & $4.97 \mathrm{E}-01$ & $8.17 \mathrm{E}+11$ \\
\hline 7 & 19 & 226.132 & $1.00 \mathrm{E}+00$ & $0.00 \mathrm{E}+00$ \\
\hline
\end{tabular}




\section{Continued}

\begin{tabular}{|c|c|c|c|c|}
\hline 7 & 20 & 223.817 & $1.00 \mathrm{E}+00$ & $0.00 \mathrm{E}+00$ \\
\hline 7 & 21 & 215.42 & $1.00 \mathrm{E}+00$ & $0.00 \mathrm{E}+00$ \\
\hline 7 & 22 & 209.79 & $1.00 \mathrm{E}+00$ & $0.00 \mathrm{E}+00$ \\
\hline 7 & 23 & 189.651 & $1.00 \mathrm{E}+00$ & $0.00 \mathrm{E}+00$ \\
\hline 7 & 24 & 163.228 & $1.00 \mathrm{E}+00$ & $0.00 \mathrm{E}+00$ \\
\hline 7 & 25 & 160.648 & $1.00 \mathrm{E}+00$ & $0.00 \mathrm{E}+00$ \\
\hline 7 & 26 & 159.223 & $1.00 \mathrm{E}+00$ & $0.00 \mathrm{E}+00$ \\
\hline 7 & 27 & 145.156 & $1.00 \mathrm{E}+00$ & $0.00 \mathrm{E}+00$ \\
\hline 7 & 28 & 41.584 & $1.00 \mathrm{E}+00$ & $0.00 \mathrm{E}+00$ \\
\hline 7 & 29 & 41.584 & $1.00 \mathrm{E}+00$ & $0.00 \mathrm{E}+00$ \\
\hline 7 & 36 & 38.897 & $1.00 \mathrm{E}+00$ & $0.00 \mathrm{E}+00$ \\
\hline 7 & 39 & 37.158 & $1.00 \mathrm{E}+00$ & $0.00 \mathrm{E}+00$ \\
\hline 7 & 40 & 37.045 & $1.00 \mathrm{E}+00$ & $0.00 \mathrm{E}+00$ \\
\hline 7 & 41 & 37.03 & $1.00 \mathrm{E}+00$ & $0.00 \mathrm{E}+00$ \\
\hline 7 & 44 & 36.94 & $1.00 \mathrm{E}+00$ & $0.00 \mathrm{E}+00$ \\
\hline 7 & 45 & 36.889 & $1.00 \mathrm{E}+00$ & $0.00 \mathrm{E}+00$ \\
\hline 7 & 48 & 36.642 & $1.00 \mathrm{E}+00$ & $0.00 \mathrm{E}+00$ \\
\hline 7 & 58 & 34.856 & $1.00 \mathrm{E}+00$ & $0.00 \mathrm{E}+00$ \\
\hline 7 & 59 & 34.819 & $1.00 \mathrm{E}+00$ & $0.00 \mathrm{E}+00$ \\
\hline 7 & 60 & 34.768 & $1.00 \mathrm{E}+00$ & $0.00 \mathrm{E}+00$ \\
\hline 7 & 61 & 34.649 & $1.00 \mathrm{E}+00$ & $0.00 \mathrm{E}+00$ \\
\hline 8 & 18 & 241.207 & $1.00 \mathrm{E}+00$ & $0.00 \mathrm{E}+00$ \\
\hline 8 & 19 & 240.967 & $1.00 \mathrm{E}+00$ & $0.00 \mathrm{E}+00$ \\
\hline 8 & 20 & 238.341 & $1.00 \mathrm{E}+00$ & $0.00 \mathrm{E}+00$ \\
\hline 8 & 21 & 228.842 & $1.00 \mathrm{E}+00$ & $0.00 \mathrm{E}+00$ \\
\hline 8 & 22 & 222.498 & $1.00 \mathrm{E}+00$ & $0.00 \mathrm{E}+00$ \\
\hline 8 & 24 & 170.819 & $1.00 \mathrm{E}+00$ & $0.00 \mathrm{E}+00$ \\
\hline 8 & 25 & 167.996 & $1.00 \mathrm{E}+00$ & $0.00 \mathrm{E}+00$ \\
\hline 8 & 26 & 166.438 & $1.00 \mathrm{E}+00$ & $0.00 \mathrm{E}+00$ \\
\hline 8 & 28 & 42.06 & $1.00 \mathrm{E}+00$ & $0.00 \mathrm{E}+00$ \\
\hline 8 & 40 & 37.423 & $1.00 \mathrm{E}+00$ & $0.00 \mathrm{E}+00$ \\
\hline 8 & 41 & 37.407 & $1.00 \mathrm{E}+00$ & $0.00 \mathrm{E}+00$ \\
\hline 8 & 42 & 37.407 & $1.00 \mathrm{E}+00$ & $0.00 \mathrm{E}+00$ \\
\hline 8 & 44 & 37.315 & $1.00 \mathrm{E}+00$ & $0.00 \mathrm{E}+00$ \\
\hline 8 & 45 & 37.263 & $1.00 \mathrm{E}+00$ & $0.00 \mathrm{E}+00$ \\
\hline 8 & 58 & 35.19 & $1.00 \mathrm{E}+00$ & $0.00 \mathrm{E}+00$ \\
\hline 8 & 59 & 35.152 & $1.00 \mathrm{E}+00$ & $0.00 \mathrm{E}+00$ \\
\hline 8 & 60 & 35.1 & $1.00 \mathrm{E}+00$ & $0.00 \mathrm{E}+00$ \\
\hline 9 & 16 & 265.688 & $1.00 \mathrm{E}+00$ & $0.00 \mathrm{E}+00$ \\
\hline 9 & 17 & 257.998 & $1.00 \mathrm{E}+00$ & $0.00 \mathrm{E}+00$ \\
\hline 9 & 19 & 245.388 & $1.00 \mathrm{E}+00$ & $0.00 \mathrm{E}+00$ \\
\hline 9 & 21 & 232.825 & $1.00 \mathrm{E}+00$ & $0.00 \mathrm{E}+00$ \\
\hline 9 & 23 & 203.012 & $1.00 \mathrm{E}+00$ & $0.00 \mathrm{E}+00$ \\
\hline 9 & 24 & 173.028 & $1.00 \mathrm{E}+00$ & $0.00 \mathrm{E}+00$ \\
\hline 9 & 25 & 170.133 & $1.00 \mathrm{E}+00$ & $0.00 \mathrm{E}+00$ \\
\hline 9 & 27 & 152.855 & $1.00 \mathrm{E}+00$ & $0.00 \mathrm{E}+00$ \\
\hline 9 & 28 & 42.193 & $1.00 \mathrm{E}+00$ & $0.00 \mathrm{E}+00$ \\
\hline 9 & 29 & 42.192 & $1.00 \mathrm{E}+00$ & $0.00 \mathrm{E}+00$ \\
\hline
\end{tabular}




\section{Continued}

\begin{tabular}{|c|c|c|c|c|}
\hline 9 & 35 & 39.429 & $1.00 \mathrm{E}+00$ & $0.00 \mathrm{E}+00$ \\
\hline 9 & 36 & 39.429 & $1.00 \mathrm{E}+00$ & $0.00 \mathrm{E}+00$ \\
\hline 9 & 38 & 37.71 & $1.00 \mathrm{E}+00$ & $0.00 \mathrm{E}+00$ \\
\hline 9 & 39 & 37.643 & $1.00 \mathrm{E}+00$ & $0.00 \mathrm{E}+00$ \\
\hline 9 & 40 & 37.528 & $1.00 \mathrm{E}+00$ & $0.00 \mathrm{E}+00$ \\
\hline 9 & 44 & 37.42 & $8.28 \mathrm{E}-01$ & $1.31 \mathrm{E}+12$ \\
\hline 9 & 48 & 37.114 & $2.71 \mathrm{E}-01$ & $4.37 \mathrm{E}+11$ \\
\hline 9 & 58 & 35.283 & $3.86 \mathrm{E}-03$ & $6.90 \mathrm{E}+09$ \\
\hline 9 & 59 & 35.245 & $3.79 \mathrm{E}-04$ & $6.79 \mathrm{E}+08$ \\
\hline 9 & 61 & 35.071 & $1.37 \mathrm{E}-04$ & $2.47 \mathrm{E}+08$ \\
\hline 10 & 17 & 272.745 & $4.71 \mathrm{E}-02$ & $8.45 E+08$ \\
\hline 10 & 19 & 258.691 & $2.38 \mathrm{E}-01$ & $4.74 \mathrm{E}+09$ \\
\hline 10 & 20 & 255.667 & $1.49 \mathrm{E}-02$ & $3.03 E+08$ \\
\hline 10 & 21 & 244.768 & $2.19 \mathrm{E}-05$ & $4.88 \mathrm{E}+05$ \\
\hline 10 & 22 & 237.525 & $6.84 \mathrm{E}-01$ & $1.62 \mathrm{E}+10$ \\
\hline 10 & 23 & 212.033 & $1.79 \mathrm{E}-04$ & $5.31 \mathrm{E}+06$ \\
\hline 10 & 24 & 179.539 & 8.63E-04 & $3.57 \mathrm{E}+07$ \\
\hline 10 & 25 & 176.423 & $1.21 \mathrm{E}-02$ & $5.17 \mathrm{E}+08$ \\
\hline 10 & 26 & 174.706 & $3.28 \mathrm{E}-04$ & $1.43 \mathrm{E}+07$ \\
\hline 10 & 27 & 157.914 & $2.16 \mathrm{E}-03$ & $1.16 \mathrm{E}+08$ \\
\hline 10 & 28 & 42.569 & $1.61 \mathrm{E}-01$ & $1.18 \mathrm{E}+11$ \\
\hline 10 & 29 & 42.569 & $1.30 \mathrm{E}-01$ & $9.57 \mathrm{E}+10$ \\
\hline 10 & 36 & 39.758 & $2.55 \mathrm{E}-04$ & $2.15 E+08$ \\
\hline 10 & 39 & 37.942 & $1.02 \mathrm{E}-01$ & $9.47 \mathrm{E}+10$ \\
\hline 10 & 40 & 37.825 & $4.59 \mathrm{E}-01$ & $4.28 \mathrm{E}+11$ \\
\hline 10 & 41 & 37.809 & $4.09 \mathrm{E}-03$ & $3.81 \mathrm{E}+09$ \\
\hline 10 & 44 & 37.715 & $4.33 \mathrm{E}-02$ & $4.06 \mathrm{E}+10$ \\
\hline 10 & 45 & 37.662 & $1.30 \mathrm{E}+00$ & $1.22 \mathrm{E}+12$ \\
\hline 10 & 48 & 37.405 & $2.62 \mathrm{E}-03$ & $2.50 \mathrm{E}+09$ \\
\hline 10 & 58 & 35.546 & $2.52 \mathrm{E}-04$ & $2.66 \mathrm{E}+08$ \\
\hline 10 & 59 & 35.507 & $2.21 \mathrm{E}-03$ & $2.33 \mathrm{E}+09$ \\
\hline 10 & 60 & 35.454 & $8.81 \mathrm{E}-04$ & $9.35 \mathrm{E}+08$ \\
\hline 10 & 61 & 35.331 & $3.66 \mathrm{E}-04$ & $3.92 \mathrm{E}+08$ \\
\hline 11 & 17 & 332.918 & $7.31 \mathrm{E}-03$ & $4.40 \mathrm{E}+08$ \\
\hline 11 & 23 & 246.696 & $1.77 \mathrm{E}-01$ & $1.94 \mathrm{E}+10$ \\
\hline 11 & 27 & 176.371 & $1.57 \mathrm{E}-02$ & $3.37 \mathrm{E}+09$ \\
\hline 11 & 29 & 43.805 & $4.69 \mathrm{E}-02$ & $1.63 E+11$ \\
\hline 11 & 36 & 40.834 & $1.52 \mathrm{E}-02$ & $6.10 \mathrm{E}+10$ \\
\hline 11 & 39 & 38.921 & $6.79 \mathrm{E}-03$ & $2.99 \mathrm{E}+10$ \\
\hline 11 & 48 & 38.356 & $3.05 \mathrm{E}-01$ & $1.39 \mathrm{E}+12$ \\
\hline 11 & 61 & 36.178 & $8.11 \mathrm{E}-02$ & $4.13 \mathrm{E}+11$ \\
\hline 12 & 16 & 399.812 & $2.20 \mathrm{E}-05$ & $3.06 \mathrm{E}+05$ \\
\hline 12 & 17 & 382.649 & $7.62 \mathrm{E}-05$ & $1.16 \mathrm{E}+06$ \\
\hline 12 & 19 & 355.55 & $7.16 \mathrm{E}-04$ & $1.26 \mathrm{E}+07$ \\
\hline 12 & 21 & 329.769 & $2.25 \mathrm{E}-04$ & $4.61 \mathrm{E}+06$ \\
\hline 12 & 23 & 272.986 & $3.40 \mathrm{E}-03$ & $1.01 \mathrm{E}+08$ \\
\hline 12 & 24 & 221.398 & $5.45 \mathrm{E}-01$ & $2.47 \mathrm{E}+10$ \\
\hline 12 & 25 & 216.679 & $1.74 \mathrm{E}-03$ & $8.25 \mathrm{E}+07$ \\
\hline
\end{tabular}




\section{Continued}

\begin{tabular}{|c|c|c|c|c|}
\hline 12 & 27 & 189.412 & $1.20 \mathrm{E}-01$ & $7.46 \mathrm{E}+09$ \\
\hline 12 & 28 & 44.567 & $1.93 \mathrm{E}-04$ & $2.16 \mathrm{E}+08$ \\
\hline 12 & 29 & 44.567 & $1.95 \mathrm{E}-03$ & $2.18 \mathrm{E}+09$ \\
\hline 12 & 35 & 41.495 & $6.28 \mathrm{E}-02$ & $8.12 \mathrm{E}+10$ \\
\hline 12 & 36 & 41.495 & $1.08 \mathrm{E}-01$ & $1.40 \mathrm{E}+11$ \\
\hline 12 & 38 & 39.595 & $1.32 \mathrm{E}-04$ & $1.88 \mathrm{E}+08$ \\
\hline 12 & 39 & 39.521 & $1.24 \mathrm{E}-04$ & $1.76 \mathrm{E}+08$ \\
\hline 12 & 40 & 39.394 & $1.85 \mathrm{E}-03$ & $2.65 \mathrm{E}+09$ \\
\hline 12 & 44 & 39.275 & $3.40 \mathrm{E}-03$ & $4.90 \mathrm{E}+09$ \\
\hline 12 & 48 & 38.939 & $4.54 \mathrm{E}-06$ & $6.65 \mathrm{E}+06$ \\
\hline 12 & 58 & 36.928 & $9.59 \mathrm{E}-01$ & $1.57 \mathrm{E}+12$ \\
\hline 12 & 59 & 36.886 & $1.00 \mathrm{E}+00$ & $0.00 \mathrm{E}+00$ \\
\hline 12 & 61 & 36.696 & $1.00 \mathrm{E}+00$ & $0.00 \mathrm{E}+00$ \\
\hline 13 & 16 & 481.452 & $1.00 \mathrm{E}+00$ & $0.00 \mathrm{E}+00$ \\
\hline 13 & 17 & 456.781 & $1.00 \mathrm{E}+00$ & $0.00 \mathrm{E}+00$ \\
\hline 13 & 19 & 418.687 & $1.00 \mathrm{E}+00$ & $0.00 \mathrm{E}+00$ \\
\hline 13 & 21 & 383.391 & $1.00 \mathrm{E}+00$ & $0.00 \mathrm{E}+00$ \\
\hline 13 & 23 & 308.732 & $1.00 \mathrm{E}+00$ & $0.00 \mathrm{E}+00$ \\
\hline 13 & 24 & 244.342 & $1.00 \mathrm{E}+00$ & $0.00 \mathrm{E}+00$ \\
\hline 13 & 25 & 238.607 & $1.00 \mathrm{E}+00$ & $0.00 \mathrm{E}+00$ \\
\hline 13 & 27 & 205.958 & $1.00 \mathrm{E}+00$ & $0.00 \mathrm{E}+00$ \\
\hline 13 & 28 & 45.426 & $1.00 \mathrm{E}+00$ & $0.00 \mathrm{E}+00$ \\
\hline 13 & 29 & 45.425 & $1.00 \mathrm{E}+00$ & $0.00 \mathrm{E}+00$ \\
\hline 13 & 35 & 42.239 & $1.00 \mathrm{E}+00$ & $0.00 \mathrm{E}+00$ \\
\hline 13 & 36 & 42.238 & $1.00 \mathrm{E}+00$ & $0.00 \mathrm{E}+00$ \\
\hline 13 & 38 & 40.272 & $1.00 \mathrm{E}+00$ & $0.00 \mathrm{E}+00$ \\
\hline 13 & 39 & 40.195 & $1.00 \mathrm{E}+00$ & $0.00 \mathrm{E}+00$ \\
\hline 13 & 40 & 40.064 & $1.00 \mathrm{E}+00$ & $0.00 \mathrm{E}+00$ \\
\hline 13 & 44 & 39.941 & $1.00 \mathrm{E}+00$ & $0.00 \mathrm{E}+00$ \\
\hline 13 & 48 & 39.593 & $1.00 \mathrm{E}+00$ & $0.00 \mathrm{E}+00$ \\
\hline 13 & 58 & 37.516 & $1.00 \mathrm{E}+00$ & $0.00 \mathrm{E}+00$ \\
\hline 13 & 59 & 37.472 & $1.00 \mathrm{E}+00$ & $0.00 \mathrm{E}+00$ \\
\hline 13 & 61 & 37.276 & $1.00 \mathrm{E}+00$ & $0.00 \mathrm{E}+00$ \\
\hline 14 & 17 & 464.92 & $1.00 \mathrm{E}+00$ & $0.00 \mathrm{E}+00$ \\
\hline 14 & 19 & 425.515 & $1.00 \mathrm{E}+00$ & $0.00 \mathrm{E}+00$ \\
\hline 14 & 20 & 417.393 & $1.00 \mathrm{E}+00$ & $0.00 \mathrm{E}+00$ \\
\hline 14 & 21 & 389.109 & $1.00 \mathrm{E}+00$ & $0.00 \mathrm{E}+00$ \\
\hline 14 & 22 & 371.118 & $1.00 \mathrm{E}+00$ & $0.00 \mathrm{E}+00$ \\
\hline 14 & 23 & 312.428 & $1.00 \mathrm{E}+00$ & $0.00 \mathrm{E}+00$ \\
\hline 14 & 24 & 246.651 & $1.00 \mathrm{E}+00$ & $0.00 \mathrm{E}+00$ \\
\hline 14 & 25 & 240.809 & $1.00 \mathrm{E}+00$ & $0.00 \mathrm{E}+00$ \\
\hline 14 & 26 & 237.621 & $1.00 \mathrm{E}+00$ & $0.00 \mathrm{E}+00$ \\
\hline 14 & 27 & 207.596 & $1.00 \mathrm{E}+00$ & $0.00 \mathrm{E}+00$ \\
\hline 14 & 28 & 45.505 & $1.00 \mathrm{E}+00$ & $0.00 \mathrm{E}+00$ \\
\hline 14 & 29 & 45.505 & $1.00 \mathrm{E}+00$ & $0.00 \mathrm{E}+00$ \\
\hline 14 & 36 & 42.307 & $1.00 \mathrm{E}+00$ & $0.00 \mathrm{E}+00$ \\
\hline 14 & 39 & 40.257 & $1.00 \mathrm{E}+00$ & $0.00 \mathrm{E}+00$ \\
\hline 14 & 40 & 40.125 & $1.00 \mathrm{E}+00$ & $0.00 \mathrm{E}+00$ \\
\hline
\end{tabular}




\section{Continued}

\begin{tabular}{|c|c|c|c|c|}
\hline 14 & 41 & 40.108 & $1.00 \mathrm{E}+00$ & $0.00 \mathrm{E}+00$ \\
\hline 14 & 44 & 40.002 & $1.00 \mathrm{E}+00$ & $0.00 \mathrm{E}+00$ \\
\hline 14 & 45 & 39.942 & $1.00 \mathrm{E}+00$ & $0.00 \mathrm{E}+00$ \\
\hline 14 & 48 & 39.653 & $1.00 \mathrm{E}+00$ & $0.00 \mathrm{E}+00$ \\
\hline 14 & 58 & 37.57 & $1.00 \mathrm{E}+00$ & $0.00 \mathrm{E}+00$ \\
\hline 14 & 59 & 37.526 & $1.00 \mathrm{E}+00$ & $0.00 \mathrm{E}+00$ \\
\hline 14 & 60 & 37.468 & $1.00 \mathrm{E}+00$ & $0.00 \mathrm{E}+00$ \\
\hline 14 & 61 & 37.33 & $1.00 \mathrm{E}+00$ & $0.00 \mathrm{E}+00$ \\
\hline 15 & 17 & 879.043 & $1.00 \mathrm{E}+00$ & $0.00 \mathrm{E}+00$ \\
\hline 15 & 23 & 457.159 & $1.00 \mathrm{E}+00$ & $0.00 \mathrm{E}+00$ \\
\hline 15 & 27 & 262.9 & $1.00 \mathrm{E}+00$ & $0.00 \mathrm{E}+00$ \\
\hline 15 & 29 & 47.704 & $1.00 \mathrm{E}+00$ & $0.00 \mathrm{E}+00$ \\
\hline 15 & 36 & 44.202 & $1.00 \mathrm{E}+00$ & $0.00 \mathrm{E}+00$ \\
\hline 15 & 39 & 41.969 & $1.00 \mathrm{E}+00$ & $0.00 \mathrm{E}+00$ \\
\hline 15 & 48 & 41.313 & $1.00 \mathrm{E}+00$ & $0.00 \mathrm{E}+00$ \\
\hline 15 & 61 & 38.797 & $1.00 \mathrm{E}+00$ & $0.00 \mathrm{E}+00$ \\
\hline 16 & 30 & 47.229 & $1.00 \mathrm{E}+00$ & $0.00 \mathrm{E}+00$ \\
\hline 16 & 33 & 46.808 & $7.00 \mathrm{E}-05$ & $7.11 \mathrm{E}+07$ \\
\hline 16 & 43 & 43.672 & $2.23 \mathrm{E}-05$ & $2.60 \mathrm{E}+07$ \\
\hline 16 & 46 & 43.374 & $1.69 \mathrm{E}-04$ & $2.00 \mathrm{E}+08$ \\
\hline 16 & 50 & 42.331 & $9.38 \mathrm{E}-01$ & $1.16 \mathrm{E}+12$ \\
\hline 17 & 30 & 47.481 & $6.07 \mathrm{E}-02$ & $5.98 \mathrm{E}+10$ \\
\hline 17 & 31 & 47.365 & $7.41 \mathrm{E}-03$ & $4.41 \mathrm{E}+09$ \\
\hline 17 & 33 & 47.055 & $1.73 \mathrm{E}-03$ & $1.73 \mathrm{E}+09$ \\
\hline 17 & 34 & 46.927 & $1.21 \mathrm{E}-02$ & $7.35 \mathrm{E}+09$ \\
\hline 17 & 37 & 46.183 & $1.82 \mathrm{E}-03$ & $5.68 \mathrm{E}+09$ \\
\hline 17 & 43 & 43.887 & $3.91 \mathrm{E}-05$ & $4.51 \mathrm{E}+07$ \\
\hline 17 & 46 & 43.586 & $2.55 E-06$ & $2.98 E+06$ \\
\hline 17 & 47 & 43.549 & $2.72 \mathrm{E}-04$ & $1.91 \mathrm{E}+08$ \\
\hline 17 & 49 & 43.276 & $3.52 \mathrm{E}-04$ & $1.26 \mathrm{E}+09$ \\
\hline 17 & 50 & 42.533 & $8.93 \mathrm{E}-01$ & $1.10 \mathrm{E}+12$ \\
\hline 17 & 51 & 42.497 & $1.71 \mathrm{E}+00$ & $1.27 \mathrm{E}+12$ \\
\hline 17 & 55 & 42.411 & $1.71 \mathrm{E}-01$ & $1.27 \mathrm{E}+11$ \\
\hline 17 & 63 & 39.583 & $3.65 \mathrm{E}-02$ & $3.10 \mathrm{E}+10$ \\
\hline 18 & 32 & 47.544 & $2.61 \mathrm{E}-01$ & $1.10 \mathrm{E}+11$ \\
\hline 18 & 52 & 42.835 & $2.39 \mathrm{E}-01$ & $9.66 \mathrm{E}+10$ \\
\hline 18 & 53 & 42.823 & $7.38 \mathrm{E}+00$ & $2.44 \mathrm{E}+12$ \\
\hline 18 & 54 & 42.778 & $5.61 \mathrm{E}-02$ & $2.92 \mathrm{E}+10$ \\
\hline 18 & 56 & 42.749 & $1.10 \mathrm{E}-02$ & $5.72 \mathrm{E}+09$ \\
\hline 18 & 57 & 42.728 & 7.67E-01 & $3.12 \mathrm{E}+11$ \\
\hline 18 & 62 & 39.912 & $4.18 \mathrm{E}-06$ & $2.50 \mathrm{E}+06$ \\
\hline 18 & 64 & 39.89 & $3.73 \mathrm{E}-05$ & $2.23 \mathrm{E}+07$ \\
\hline 18 & 65 & 39.887 & $2.81 \mathrm{E}-04$ & $1.31 \mathrm{E}+08$ \\
\hline 19 & 30 & 47.934 & $3.47 \mathrm{E}-02$ & $3.36 \mathrm{E}+10$ \\
\hline 19 & 31 & 47.817 & $1.18 \mathrm{E}-02$ & $6.89 \mathrm{E}+09$ \\
\hline 19 & 32 & 47.553 & $5.45 \mathrm{E}-03$ & $2.30 \mathrm{E}+09$ \\
\hline 19 & 33 & 47.5 & $2.94 \mathrm{E}-02$ & $2.89 \mathrm{E}+10$ \\
\hline 19 & 34 & 47.37 & $6.11 \mathrm{E}-02$ & $3.63 E+10$ \\
\hline
\end{tabular}




\section{Continued}

\begin{tabular}{|c|c|c|c|c|}
\hline 19 & 43 & 44.274 & $7.21 \mathrm{E}-06$ & $8.18 \mathrm{E}+06$ \\
\hline 19 & 46 & 43.967 & $1.71 \mathrm{E}-03$ & $1.97 \mathrm{E}+09$ \\
\hline 19 & 47 & 43.931 & $4.81 \mathrm{E}-04$ & $3.32 \mathrm{E}+08$ \\
\hline 19 & 50 & 42.896 & $9.75 \mathrm{E}-02$ & $1.18 \mathrm{E}+11$ \\
\hline 19 & 51 & 42.859 & $1.07 \mathrm{E}+00$ & $7.78 \mathrm{E}+11$ \\
\hline 19 & 54 & 42.785 & $3.30 \mathrm{E}+00$ & $1.72 \mathrm{E}+12$ \\
\hline 19 & 55 & 42.773 & $3.42 \mathrm{E}-02$ & $2.49 \mathrm{E}+10$ \\
\hline 19 & 56 & 42.757 & $9.02 \mathrm{E}-02$ & $4.70 \mathrm{E}+10$ \\
\hline 19 & 62 & 39.918 & $8.24 \mathrm{E}-04$ & $4.93 \mathrm{E}+08$ \\
\hline 19 & 63 & 39.898 & $5.92 \mathrm{E}-03$ & $4.96 \mathrm{E}+09$ \\
\hline 19 & 64 & 39.897 & $1.02 \mathrm{E}-01$ & $6.08 \mathrm{E}+10$ \\
\hline 20 & 31 & 47.921 & $1.75 \mathrm{E}-01$ & $1.02 \mathrm{E}+11$ \\
\hline 20 & 32 & 47.657 & $2.75 \mathrm{E}-02$ & $1.15 \mathrm{E}+10$ \\
\hline 20 & 34 & 47.473 & $5.92 \mathrm{E}-03$ & $3.50 \mathrm{E}+09$ \\
\hline 20 & 47 & 44.019 & $3.69 \mathrm{E}-04$ & $2.54 \mathrm{E}+08$ \\
\hline 20 & 51 & 42.944 & $2.32 \mathrm{E}-02$ & $1.68 \mathrm{E}+10$ \\
\hline 20 & 52 & 42.927 & $5.56 \mathrm{E}+00$ & $2.24 \mathrm{E}+12$ \\
\hline 20 & 54 & 42.869 & $2.06 \mathrm{E}-01$ & $1.07 \mathrm{E}+11$ \\
\hline 20 & 55 & 42.857 & $3.87 \mathrm{E}-02$ & $2.81 \mathrm{E}+10$ \\
\hline 20 & 56 & 42.841 & $6.68 \mathrm{E}-01$ & $3.47 \mathrm{E}+11$ \\
\hline 20 & 57 & 42.819 & $8.97 \mathrm{E}-02$ & $3.63 \mathrm{E}+10$ \\
\hline 20 & 62 & 39.991 & $6.28 \mathrm{E}-04$ & $3.74 \mathrm{E}+08$ \\
\hline 20 & 63 & 39.971 & $2.17 \mathrm{E}-04$ & $1.81 \mathrm{E}+08$ \\
\hline 20 & 64 & 39.97 & $1.00 \mathrm{E}+00$ & $0.00 \mathrm{E}+00$ \\
\hline 20 & 65 & 39.966 & $1.00 \mathrm{E}+00$ & $0.00 \mathrm{E}+00$ \\
\hline 21 & 30 & 48.445 & $1.00 \mathrm{E}+00$ & $0.00 \mathrm{E}+00$ \\
\hline 21 & 31 & 48.325 & $1.00 \mathrm{E}+00$ & $0.00 \mathrm{E}+00$ \\
\hline 21 & 32 & 48.056 & $1.00 \mathrm{E}+00$ & $0.00 \mathrm{E}+00$ \\
\hline 21 & 33 & 48.001 & $1.00 \mathrm{E}+00$ & $0.00 \mathrm{E}+00$ \\
\hline 21 & 34 & 47.868 & $1.00 \mathrm{E}+00$ & $0.00 \mathrm{E}+00$ \\
\hline 21 & 43 & 44.709 & $1.00 \mathrm{E}+00$ & $0.00 \mathrm{E}+00$ \\
\hline 21 & 46 & 44.397 & $1.00 \mathrm{E}+00$ & $0.00 \mathrm{E}+00$ \\
\hline 21 & 47 & 44.359 & $1.00 \mathrm{E}+00$ & $0.00 \mathrm{E}+00$ \\
\hline 21 & 50 & 43.304 & $1.00 \mathrm{E}+00$ & $0.00 \mathrm{E}+00$ \\
\hline 21 & 51 & 43.267 & $1.00 \mathrm{E}+00$ & $0.00 \mathrm{E}+00$ \\
\hline 21 & 54 & 43.192 & $1.00 \mathrm{E}+00$ & $0.00 \mathrm{E}+00$ \\
\hline 21 & 55 & 43.179 & $1.00 \mathrm{E}+00$ & $0.00 \mathrm{E}+00$ \\
\hline 21 & 56 & 43.163 & $1.00 \mathrm{E}+00$ & $0.00 \mathrm{E}+00$ \\
\hline 21 & 62 & 40.272 & $1.00 \mathrm{E}+00$ & $0.00 \mathrm{E}+00$ \\
\hline 21 & 63 & 40.251 & $1.00 \mathrm{E}+00$ & $0.00 \mathrm{E}+00$ \\
\hline 21 & 64 & 40.25 & $1.00 \mathrm{E}+00$ & $0.00 \mathrm{E}+00$ \\
\hline 22 & 31 & 48.617 & $1.00 \mathrm{E}+00$ & $0.00 \mathrm{E}+00$ \\
\hline 22 & 32 & 48.345 & $1.00 \mathrm{E}+00$ & $0.00 \mathrm{E}+00$ \\
\hline 22 & 34 & 48.156 & $1.00 \mathrm{E}+00$ & $0.00 \mathrm{E}+00$ \\
\hline 22 & 47 & 44.606 & $1.00 \mathrm{E}+00$ & $0.00 \mathrm{E}+00$ \\
\hline 22 & 51 & 43.502 & $1.00 \mathrm{E}+00$ & $0.00 \mathrm{E}+00$ \\
\hline 22 & 52 & 43.485 & $1.00 \mathrm{E}+00$ & $0.00 \mathrm{E}+00$ \\
\hline 22 & 54 & 43.425 & $1.00 \mathrm{E}+00$ & $0.00 \mathrm{E}+00$ \\
\hline
\end{tabular}




\section{Continued}

\begin{tabular}{|c|c|c|c|c|}
\hline 22 & 55 & 43.412 & $1.00 \mathrm{E}+00$ & $0.00 \mathrm{E}+00$ \\
\hline 22 & 56 & 43.396 & $1.00 \mathrm{E}+00$ & $0.00 \mathrm{E}+00$ \\
\hline 22 & 57 & 43.374 & $1.00 \mathrm{E}+00$ & $0.00 \mathrm{E}+00$ \\
\hline 22 & 62 & 40.475 & $1.00 \mathrm{E}+00$ & $0.00 \mathrm{E}+00$ \\
\hline 22 & 63 & 40.454 & $1.00 \mathrm{E}+00$ & $0.00 \mathrm{E}+00$ \\
\hline 22 & 64 & 40.453 & $1.00 \mathrm{E}+00$ & $0.00 \mathrm{E}+00$ \\
\hline 22 & 65 & 40.449 & $1.00 \mathrm{E}+00$ & $0.00 \mathrm{E}+00$ \\
\hline 23 & 30 & 49.972 & $1.00 \mathrm{E}+00$ & $0.00 \mathrm{E}+00$ \\
\hline 23 & 31 & 49.844 & $1.00 \mathrm{E}+00$ & $0.00 \mathrm{E}+00$ \\
\hline 23 & 33 & 49.5 & $1.00 \mathrm{E}+00$ & $0.00 \mathrm{E}+00$ \\
\hline 23 & 34 & 49.359 & $1.00 \mathrm{E}+00$ & $0.00 \mathrm{E}+00$ \\
\hline 23 & 37 & 48.536 & $1.00 \mathrm{E}+00$ & $0.00 \mathrm{E}+00$ \\
\hline 23 & 43 & 46.006 & $1.00 \mathrm{E}+00$ & $0.00 \mathrm{E}+00$ \\
\hline 23 & 46 & 45.676 & $1.00 \mathrm{E}+00$ & $0.00 \mathrm{E}+00$ \\
\hline 23 & 47 & 45.636 & $1.00 \mathrm{E}+00$ & $0.00 \mathrm{E}+00$ \\
\hline 23 & 49 & 45.335 & $1.00 \mathrm{E}+00$ & $0.00 \mathrm{E}+00$ \\
\hline 23 & 50 & 44.521 & $1.00 \mathrm{E}+00$ & $0.00 \mathrm{E}+00$ \\
\hline 23 & 51 & 44.481 & $1.00 \mathrm{E}+00$ & $0.00 \mathrm{E}+00$ \\
\hline 23 & 55 & 44.388 & $1.00 \mathrm{E}+00$ & $0.00 \mathrm{E}+00$ \\
\hline 23 & 63 & 41.299 & $1.00 \mathrm{E}+00$ & $0.00 \mathrm{E}+00$ \\
\hline 24 & 30 & 52.198 & $1.00 \mathrm{E}+00$ & $0.00 \mathrm{E}+00$ \\
\hline 24 & 31 & 52.059 & $1.00 \mathrm{E}+00$ & $0.00 \mathrm{E}+00$ \\
\hline 24 & 32 & 51.747 & $1.00 \mathrm{E}+00$ & $0.00 \mathrm{E}+00$ \\
\hline 24 & 33 & 51.684 & $1.00 \mathrm{E}+00$ & $0.00 \mathrm{E}+00$ \\
\hline 24 & 34 & 51.53 & $1.00 \mathrm{E}+00$ & $0.00 \mathrm{E}+00$ \\
\hline 24 & 43 & 47.887 & $1.00 \mathrm{E}+00$ & $0.00 \mathrm{E}+00$ \\
\hline 24 & 46 & 47.529 & $1.00 \mathrm{E}+00$ & $0.00 \mathrm{E}+00$ \\
\hline 24 & 47 & 47.486 & $1.00 \mathrm{E}+00$ & $0.00 \mathrm{E}+00$ \\
\hline 24 & 50 & 46.279 & $1.00 \mathrm{E}+00$ & $0.00 \mathrm{E}+00$ \\
\hline 24 & 51 & 46.237 & $7.00 \mathrm{E}-05$ & $4.37 \mathrm{E}+07$ \\
\hline 24 & 54 & 46.151 & $2.64 \mathrm{E}-03$ & $1.18 \mathrm{E}+09$ \\
\hline 24 & 55 & 46.136 & $8.18 \mathrm{E}-03$ & $5.13 \mathrm{E}+09$ \\
\hline 24 & 56 & 46.117 & $3.39 \mathrm{E}-02$ & $1.52 \mathrm{E}+10$ \\
\hline 24 & 62 & 42.832 & $4.35 \mathrm{E}+00$ & $2.26 \mathrm{E}+12$ \\
\hline 24 & 63 & 42.808 & $3.25 \mathrm{E}-01$ & $2.37 \mathrm{E}+11$ \\
\hline 24 & 64 & 42.807 & $7.78 \mathrm{E}-03$ & $4.05 E+09$ \\
\hline 25 & 30 & 52.468 & $6.22 \mathrm{E}-04$ & $5.02 \mathrm{E}+08$ \\
\hline 25 & 31 & 52.327 & $7.78 \mathrm{E}-04$ & $3.79 E+08$ \\
\hline 25 & 32 & 52.012 & $7.00 \mathrm{E}-04$ & $2.47 \mathrm{E}+08$ \\
\hline 25 & 33 & 51.948 & $4.94 \mathrm{E}-04$ & $4.07 \mathrm{E}+08$ \\
\hline 25 & 34 & 51.792 & $2.49 \mathrm{E}-03$ & $1.24 \mathrm{E}+09$ \\
\hline 25 & 43 & 48.113 & $2.31 \mathrm{E}-04$ & $2.21 \mathrm{E}+08$ \\
\hline 25 & 46 & 47.752 & $1.33 \mathrm{E}-01$ & $1.29 \mathrm{E}+11$ \\
\hline 25 & 47 & 47.708 & $1.31 \mathrm{E}-02$ & $7.66 \mathrm{E}+09$ \\
\hline 25 & 50 & 46.491 & $3.61 \mathrm{E}-03$ & $3.72 \mathrm{E}+09$ \\
\hline 25 & 51 & 46.448 & $3.40 \mathrm{E}-02$ & $2.11 \mathrm{E}+10$ \\
\hline 25 & 54 & 46.361 & $7.57 \mathrm{E}-02$ & $3.35 \mathrm{E}+10$ \\
\hline 25 & 55 & 46.346 & $1.88 \mathrm{E}-03$ & $1.17 \mathrm{E}+09$ \\
\hline
\end{tabular}




\section{Continued}

\begin{tabular}{|c|c|c|c|c|}
\hline 25 & 56 & 46.328 & $9.12 \mathrm{E}-05$ & $4.05 \mathrm{E}+07$ \\
\hline 25 & 62 & 43.013 & $4.50 \mathrm{E}-02$ & $2.32 \mathrm{E}+10$ \\
\hline 25 & 63 & 42.989 & $1.89 \mathrm{E}-01$ & $1.37 \mathrm{E}+11$ \\
\hline 25 & 64 & 42.988 & $4.38 \mathrm{E}+00$ & $2.25 \mathrm{E}+12$ \\
\hline 26 & 31 & 52.48 & $8.45 \mathrm{E}-04$ & $4.09 \mathrm{E}+08$ \\
\hline 26 & 32 & 52.163 & $9.57 \mathrm{E}-04$ & $3.35 \mathrm{E}+08$ \\
\hline 26 & 34 & 51.942 & $2.99 \mathrm{E}-04$ & $1.47 \mathrm{E}+08$ \\
\hline 26 & 47 & 47.836 & $2.11 \mathrm{E}-01$ & $1.23 \mathrm{E}+11$ \\
\hline 26 & 51 & 46.568 & $9.31 \mathrm{E}-04$ & $5.72 \mathrm{E}+08$ \\
\hline 26 & 52 & 46.549 & $2.22 \mathrm{E}-02$ & $7.61 \mathrm{E}+09$ \\
\hline 26 & 54 & 46.481 & $1.17 \mathrm{E}-02$ & $5.18 \mathrm{E}+09$ \\
\hline 26 & 55 & 46.466 & $4.83 \mathrm{E}-04$ & $2.98 \mathrm{E}+08$ \\
\hline 26 & 56 & 46.447 & $2.64 \mathrm{E}-03$ & $1.17 \mathrm{E}+09$ \\
\hline 26 & 57 & 46.422 & $1.41 \mathrm{E}-02$ & $4.83 \mathrm{E}+09$ \\
\hline 26 & 62 & 43.116 & $2.93 \mathrm{E}-01$ & $1.50 \mathrm{E}+11$ \\
\hline 26 & 63 & 43.093 & $1.47 \mathrm{E}-02$ & $1.05 \mathrm{E}+10$ \\
\hline 26 & 64 & 43.092 & $2.26 \mathrm{E}-01$ & $1.16 \mathrm{E}+11$ \\
\hline 26 & 65 & 43.087 & $6.04 \mathrm{E}+00$ & $2.41 \mathrm{E}+12$ \\
\hline 27 & 30 & 54.363 & $6.12 \mathrm{E}-05$ & $4.61 \mathrm{E}+07$ \\
\hline 27 & 31 & 54.211 & $1.64 \mathrm{E}-05$ & $7.47 \mathrm{E}+06$ \\
\hline 27 & 33 & 53.805 & $1.07 \mathrm{E}-02$ & $8.24 \mathrm{E}+09$ \\
\hline 27 & 34 & 53.638 & $4.95 \mathrm{E}-04$ & $2.30 \mathrm{E}+08$ \\
\hline 27 & 37 & 52.667 & $2.45 \mathrm{E}-03$ & $5.89 \mathrm{E}+09$ \\
\hline 27 & 43 & 49.702 & $3.00 \mathrm{E}-02$ & $2.70 \mathrm{E}+10$ \\
\hline 27 & 46 & 49.317 & $1.60 \mathrm{E}-02$ & $1.46 \mathrm{E}+10$ \\
\hline 27 & 47 & 49.27 & $2.56 \mathrm{E}-03$ & $1.41 \mathrm{E}+09$ \\
\hline 27 & 49 & 48.92 & $5.50 \mathrm{E}-02$ & $1.53 \mathrm{E}+11$ \\
\hline 27 & 50 & 47.973 & $3.83 \mathrm{E}-03$ & $3.70 \mathrm{E}+09$ \\
\hline 27 & 51 & 47.927 & $1.34 \mathrm{E}-01$ & $7.76 \mathrm{E}+10$ \\
\hline 27 & 55 & 47.819 & $2.77 \mathrm{E}-01$ & $1.62 \mathrm{E}+11$ \\
\hline 27 & 63 & 44.253 & $2.54 \mathrm{E}+00$ & $1.73 \mathrm{E}+12$ \\
\hline 28 & 30 & 808.807 & $4.19 \mathrm{E}-01$ & $1.43 \mathrm{E}+09$ \\
\hline 28 & 31 & 776.531 & $3.44 \mathrm{E}-01$ & $7.60 \mathrm{E}+08$ \\
\hline 28 & 32 & 712.508 & $1.11 \mathrm{E}+00$ & $2.08 \mathrm{E}+09$ \\
\hline 28 & 33 & 700.677 & $8.51 \mathrm{E}-04$ & $3.85 E+06$ \\
\hline 28 & 34 & 673.407 & $1.00 \mathrm{E}+00$ & $0.00 \mathrm{E}+00$ \\
\hline 28 & 43 & 337.684 & $1.00 \mathrm{E}+00$ & $0.00 \mathrm{E}+00$ \\
\hline 28 & 46 & 320.653 & $1.00 \mathrm{E}+00$ & $0.00 \mathrm{E}+00$ \\
\hline 28 & 47 & 318.702 & $1.00 \mathrm{E}+00$ & $0.00 \mathrm{E}+00$ \\
\hline 28 & 50 & 271.244 & $1.00 \mathrm{E}+00$ & $0.00 \mathrm{E}+00$ \\
\hline 28 & 51 & 269.787 & $1.00 \mathrm{E}+00$ & $0.00 \mathrm{E}+00$ \\
\hline 28 & 54 & 266.883 & $1.00 \mathrm{E}+00$ & $0.00 \mathrm{E}+00$ \\
\hline 28 & 55 & 266.392 & $1.00 \mathrm{E}+00$ & $0.00 \mathrm{E}+00$ \\
\hline 28 & 56 & 265.781 & $1.00 \mathrm{E}+00$ & $0.00 \mathrm{E}+00$ \\
\hline 28 & 62 & 184.305 & $1.00 \mathrm{E}+00$ & $0.00 \mathrm{E}+00$ \\
\hline 28 & 63 & 183.868 & $1.00 \mathrm{E}+00$ & $0.00 \mathrm{E}+00$ \\
\hline 28 & 64 & 183.852 & $1.00 \mathrm{E}+00$ & $0.00 \mathrm{E}+00$ \\
\hline 29 & 30 & 808.987 & $1.00 \mathrm{E}+00$ & $0.00 \mathrm{E}+00$ \\
\hline
\end{tabular}




\section{Continued}

\begin{tabular}{|c|c|c|c|c|}
\hline 29 & 31 & 776.697 & $1.00 \mathrm{E}+00$ & $0.00 \mathrm{E}+00$ \\
\hline 29 & 33 & 700.812 & $1.00 \mathrm{E}+00$ & $0.00 \mathrm{E}+00$ \\
\hline 29 & 34 & 673.532 & $1.00 \mathrm{E}+00$ & $0.00 \mathrm{E}+00$ \\
\hline 29 & 37 & 546.975 & $1.00 \mathrm{E}+00$ & $0.00 \mathrm{E}+00$ \\
\hline 29 & 43 & 337.715 & $1.00 \mathrm{E}+00$ & $0.00 \mathrm{E}+00$ \\
\hline 29 & 46 & 320.682 & $1.00 \mathrm{E}+00$ & $0.00 \mathrm{E}+00$ \\
\hline 29 & 47 & 318.73 & $1.00 \mathrm{E}+00$ & $0.00 \mathrm{E}+00$ \\
\hline 29 & 49 & 304.629 & $1.00 \mathrm{E}+00$ & $0.00 \mathrm{E}+00$ \\
\hline 29 & 50 & 271.264 & $1.00 \mathrm{E}+00$ & $0.00 \mathrm{E}+00$ \\
\hline 29 & 51 & 269.807 & $1.00 \mathrm{E}+00$ & $0.00 \mathrm{E}+00$ \\
\hline 29 & 55 & 266.412 & $1.00 \mathrm{E}+00$ & $0.00 \mathrm{E}+00$ \\
\hline 29 & 63 & 183.877 & $1.00 \mathrm{E}+00$ & $0.00 \mathrm{E}+00$ \\
\hline 30 & 35 & 2354.366 & $1.00 \mathrm{E}+00$ & $0.00 \mathrm{E}+00$ \\
\hline 30 & 36 & 2353.512 & $1.00 \mathrm{E}+00$ & $0.00 \mathrm{E}+00$ \\
\hline 30 & 38 & 632.487 & $1.00 \mathrm{E}+00$ & $0.00 \mathrm{E}+00$ \\
\hline 30 & 39 & 614.122 & $1.00 \mathrm{E}+00$ & $0.00 \mathrm{E}+00$ \\
\hline 30 & 40 & 584.821 & $1.00 \mathrm{E}+00$ & $0.00 \mathrm{E}+00$ \\
\hline 30 & 44 & 559.642 & $1.00 \mathrm{E}+00$ & $0.00 \mathrm{E}+00$ \\
\hline 30 & 48 & 498.27 & $1.00 \mathrm{E}+00$ & $0.00 \mathrm{E}+00$ \\
\hline 30 & 58 & 293.664 & $1.00 \mathrm{E}+00$ & $0.00 \mathrm{E}+00$ \\
\hline 30 & 59 & 291.032 & $1.00 \mathrm{E}+00$ & $0.00 \mathrm{E}+00$ \\
\hline 30 & 61 & 279.612 & $1.00 \mathrm{E}+00$ & $0.00 \mathrm{E}+00$ \\
\hline 31 & 36 & 2677.322 & $1.00 \mathrm{E}+00$ & $0.00 \mathrm{E}+00$ \\
\hline 31 & 39 & 634.135 & $1.00 \mathrm{E}+00$ & $0.00 \mathrm{E}+00$ \\
\hline 31 & 40 & 602.942 & $1.00 \mathrm{E}+00$ & $0.00 \mathrm{E}+00$ \\
\hline 31 & 41 & 598.946 & $1.00 \mathrm{E}+00$ & $0.00 \mathrm{E}+00$ \\
\hline 31 & 44 & 576.214 & $1.00 \mathrm{E}+00$ & $0.00 \mathrm{E}+00$ \\
\hline 31 & 45 & 564.046 & $1.00 \mathrm{E}+00$ & $0.00 \mathrm{E}+00$ \\
\hline 31 & 48 & 511.364 & $1.00 \mathrm{E}+00$ & $0.00 \mathrm{E}+00$ \\
\hline 31 & 58 & 298.164 & $1.00 \mathrm{E}+00$ & $0.00 \mathrm{E}+00$ \\
\hline 31 & 59 & 295.451 & $1.00 \mathrm{E}+00$ & $0.00 \mathrm{E}+00$ \\
\hline 31 & 60 & 291.849 & $1.00 \mathrm{E}+00$ & $0.00 \mathrm{E}+00$ \\
\hline 31 & 61 & 283.688 & $1.00 \mathrm{E}+00$ & $0.00 \mathrm{E}+00$ \\
\hline 32 & 40 & 648.164 & $1.00 \mathrm{E}+00$ & $0.00 \mathrm{E}+00$ \\
\hline 32 & 41 & 643.549 & $1.00 \mathrm{E}+00$ & $0.00 \mathrm{E}+00$ \\
\hline 32 & 42 & 643.401 & $1.00 \mathrm{E}+00$ & $0.00 \mathrm{E}+00$ \\
\hline 32 & 44 & 617.378 & $1.00 \mathrm{E}+00$ & $0.00 \mathrm{E}+00$ \\
\hline 32 & 45 & 603.43 & $1.00 \mathrm{E}+00$ & $0.00 \mathrm{E}+00$ \\
\hline 32 & 58 & 308.819 & $1.00 \mathrm{E}+00$ & $0.00 \mathrm{E}+00$ \\
\hline 32 & 59 & 305.909 & $1.00 \mathrm{E}+00$ & $0.00 \mathrm{E}+00$ \\
\hline 32 & 60 & 302.05 & $1.00 \mathrm{E}+00$ & $0.00 \mathrm{E}+00$ \\
\hline 33 & 35 & 4274.59 & $6.17 \mathrm{E}-09$ & $7.51 \mathrm{E}-01$ \\
\hline 33 & 36 & 4271.77 & $1.33 \mathrm{E}-04$ & $1.62 \mathrm{E}+04$ \\
\hline 33 & 38 & 719.291 & $2.64 \mathrm{E}-04$ & $1.13 E+06$ \\
\hline 33 & 39 & 695.634 & $5.90 \mathrm{E}-03$ & $2.71 \mathrm{E}+07$ \\
\hline 33 & 40 & 658.275 & $4.79 \mathrm{E}-02$ & $2.45 \mathrm{E}+08$ \\
\hline 33 & 44 & 626.545 & $6.95 \mathrm{E}-01$ & $3.93 \mathrm{E}+09$ \\
\hline 33 & 48 & 550.617 & $2.74 \mathrm{E}-01$ & $2.01 E+09$ \\
\hline
\end{tabular}




\section{Continued}

\begin{tabular}{|c|c|c|c|c|}
\hline 33 & 58 & 311.096 & $8.49 \mathrm{E}-05$ & $1.95 \mathrm{E}+06$ \\
\hline 33 & 59 & 308.143 & $2.70 \mathrm{E}-04$ & $6.32 \mathrm{E}+06$ \\
\hline 33 & 61 & 295.37 & $3.48 \mathrm{E}-03$ & $8.87 \mathrm{E}+07$ \\
\hline 34 & 36 & 5672.15 & $5.86 \mathrm{E}-06$ & $2.43 \mathrm{E}+02$ \\
\hline 34 & 39 & 724.773 & $7.24 \mathrm{E}-02$ & $1.84 \mathrm{E}+08$ \\
\hline 34 & 40 & 684.31 & $3.50 \mathrm{E}-01$ & $9.97 \mathrm{E}+08$ \\
\hline 34 & 41 & 679.168 & $7.46 \mathrm{E}-03$ & $2.16 \mathrm{E}+07$ \\
\hline 34 & 44 & 650.085 & $4.09 \mathrm{E}-02$ & $1.29 \mathrm{E}+08$ \\
\hline 34 & 45 & 634.639 & $1.12 \mathrm{E}+00$ & $3.72 E+09$ \\
\hline 34 & 48 & 568.715 & $3.00 \mathrm{E}-03$ & $1.24 \mathrm{E}+07$ \\
\hline 34 & 58 & 316.791 & $1.07 \mathrm{E}-04$ & $1.42 \mathrm{E}+06$ \\
\hline 34 & 59 & 313.73 & $3.03 \mathrm{E}-03$ & $4.12 \mathrm{E}+07$ \\
\hline 34 & 60 & 309.672 & $4.59 \mathrm{E}-06$ & $6.39 \mathrm{E}+04$ \\
\hline 34 & 61 & 300.5 & $6.49 \mathrm{E}-04$ & $9.58 \mathrm{E}+06$ \\
\hline 35 & 43 & 769.1 & $1.52 \mathrm{E}-01$ & $5.70 \mathrm{E}+08$ \\
\hline 35 & 46 & 686.106 & $3.21 \mathrm{E}-01$ & $1.52 \mathrm{E}+09$ \\
\hline 35 & 50 & 493.685 & $7.50 \mathrm{E}-05$ & $6.84 \mathrm{E}+05$ \\
\hline 36 & 37 & 5979.9 & $6.47 \mathrm{E}-04$ & $1.21 \mathrm{E}+05$ \\
\hline 36 & 43 & 769.191 & $2.87 \mathrm{E}-01$ & $1.08 \mathrm{E}+09$ \\
\hline 36 & 46 & 686.178 & $1.69 \mathrm{E}-01$ & $7.97 \mathrm{E}+08$ \\
\hline 36 & 47 & 677.304 & $8.30 \mathrm{E}-01$ & $2.41 \mathrm{E}+09$ \\
\hline 36 & 49 & 616.648 & $1.75 \mathrm{E}-01$ & $3.07 \mathrm{E}+09$ \\
\hline 36 & 50 & 493.723 & $3.62 \mathrm{E}-05$ & $3.30 \mathrm{E}+05$ \\
\hline 36 & 51 & 488.915 & $1.35 \mathrm{E}-04$ & $7.52 \mathrm{E}+05$ \\
\hline 36 & 55 & 477.879 & $9.42 \mathrm{E}-06$ & $5.50 \mathrm{E}+04$ \\
\hline 36 & 63 & 264.732 & $4.86 \mathrm{E}-07$ & $9.25 \mathrm{E}+03$ \\
\hline 37 & 39 & 965.05 & $3.35 \mathrm{E}-03$ & $2.40 \mathrm{E}+07$ \\
\hline 37 & 48 & 706.803 & $2.81 \mathrm{E}-01$ & $3.76 \mathrm{E}+09$ \\
\hline 37 & 61 & 335.091 & $5.55 \mathrm{E}-03$ & $3.30 \mathrm{E}+08$ \\
\hline 38 & 43 & 6949.1 & $7.18 \mathrm{E}-07$ & $3.30 \mathrm{E}+01$ \\
\hline 38 & 46 & 3320.23 & $1.83 \mathrm{E}-04$ & $3.70 \mathrm{E}+04$ \\
\hline 38 & 50 & 1150.4 & $1.04 \mathrm{E}-01$ & $1.74 \mathrm{E}+08$ \\
\hline 39 & 43 & 10349.4 & $8.63 \mathrm{E}-07$ & $1.79 \mathrm{E}+01$ \\
\hline 39 & 46 & 3938.49 & $9.14 \mathrm{E}-05$ & $1.31 \mathrm{E}+04$ \\
\hline 39 & 47 & 3663.02 & $1.00 \mathrm{E}-04$ & $9.97 \mathrm{E}+03$ \\
\hline 39 & 49 & 2391.04 & $1.00 \mathrm{E}-04$ & $1.17 \mathrm{E}+05$ \\
\hline 39 & 50 & 1216.57 & $9.64 \mathrm{E}-02$ & $1.45 \mathrm{E}+08$ \\
\hline 39 & 51 & 1187.79 & $1.74 \mathrm{E}-01$ & $1.65 E+08$ \\
\hline 39 & 55 & 1124.69 & $2.94 \mathrm{E}-02$ & $3.10 \mathrm{E}+07$ \\
\hline 39 & 63 & 388.506 & $1.09 \mathrm{E}-03$ & $9.60 \mathrm{E}+06$ \\
\hline 40 & 43 & 66489.2 & $5.98 \mathrm{E}-07$ & $3.01 \mathrm{E}-01$ \\
\hline 40 & 46 & 5803.13 & $5.90 \mathrm{E}-05$ & $3.90 \mathrm{E}+03$ \\
\hline 40 & 47 & 5224.24 & $1.11 \mathrm{E}-04$ & $5.44 \mathrm{E}+03$ \\
\hline 40 & 50 & 1350.62 & $1.18 \mathrm{E}-02$ & $1.44 \mathrm{E}+07$ \\
\hline 40 & 51 & 1315.24 & $1.08 \mathrm{E}-01$ & $8.32 \mathrm{E}+07$ \\
\hline 40 & 54 & 1248.98 & $3.24 \mathrm{E}-01$ & $1.98 \mathrm{E}+08$ \\
\hline 40 & 55 & 1238.31 & $1.74 \mathrm{E}-02$ & $1.51 \mathrm{E}+07$ \\
\hline 40 & 56 & 1225.219 & $1.00 \mathrm{E}+00$ & $0.00 \mathrm{E}+00$ \\
\hline
\end{tabular}




\section{Continued}

\begin{tabular}{|c|c|c|c|c|}
\hline 40 & 62 & 403.31 & $1.00 \mathrm{E}+00$ & $0.00 \mathrm{E}+00$ \\
\hline 40 & 63 & 401.223 & $1.00 \mathrm{E}+00$ & $0.00 \mathrm{E}+00$ \\
\hline 40 & 64 & 401.15 & $1.00 \mathrm{E}+00$ & $0.00 \mathrm{E}+00$ \\
\hline 41 & 47 & 5544.735 & $1.00 \mathrm{E}+00$ & $0.00 \mathrm{E}+00$ \\
\hline 41 & 51 & 1334.66 & $1.00 \mathrm{E}+00$ & $0.00 \mathrm{E}+00$ \\
\hline 41 & 52 & 1318.726 & $1.00 \mathrm{E}+00$ & $0.00 \mathrm{E}+00$ \\
\hline 41 & 54 & 1266.483 & $1.00 \mathrm{E}+00$ & $0.00 \mathrm{E}+00$ \\
\hline 41 & 55 & 1255.512 & $1.00 \mathrm{E}+00$ & $0.00 \mathrm{E}+00$ \\
\hline 41 & 56 & 1242.057 & $1.00 \mathrm{E}+00$ & $0.00 \mathrm{E}+00$ \\
\hline 41 & 57 & 1224.336 & $1.00 \mathrm{E}+00$ & $0.00 \mathrm{E}+00$ \\
\hline 41 & 62 & 405.117 & $1.00 \mathrm{E}+00$ & $0.00 \mathrm{E}+00$ \\
\hline 41 & 63 & 403.012 & $1.00 \mathrm{E}+00$ & $0.00 \mathrm{E}+00$ \\
\hline 41 & 64 & 402.938 & $1.00 \mathrm{E}+00$ & $0.00 \mathrm{E}+00$ \\
\hline 41 & 65 & 402.571 & $1.00 \mathrm{E}+00$ & $0.00 \mathrm{E}+00$ \\
\hline 42 & 52 & 1319.348 & $1.00 \mathrm{E}+00$ & $0.00 \mathrm{E}+00$ \\
\hline 42 & 53 & 1307.749 & $1.00 \mathrm{E}+00$ & $0.00 \mathrm{E}+00$ \\
\hline 42 & 54 & 1267.057 & $1.00 \mathrm{E}+00$ & $0.00 \mathrm{E}+00$ \\
\hline 42 & 56 & 1242.608 & $1.00 \mathrm{E}+00$ & $0.00 \mathrm{E}+00$ \\
\hline 42 & 57 & 1224.871 & $1.00 \mathrm{E}+00$ & $0.00 \mathrm{E}+00$ \\
\hline 42 & 62 & 405.176 & $1.00 \mathrm{E}+00$ & $0.00 \mathrm{E}+00$ \\
\hline 42 & 64 & 402.996 & $1.00 \mathrm{E}+00$ & $0.00 \mathrm{E}+00$ \\
\hline 42 & 65 & 402.629 & $1.00 \mathrm{E}+00$ & $0.00 \mathrm{E}+00$ \\
\hline 43 & 44 & 16156.91 & $1.00 \mathrm{E}+00$ & $0.00 \mathrm{E}+00$ \\
\hline 43 & 48 & 3546.327 & $1.00 \mathrm{E}+00$ & $0.00 \mathrm{E}+00$ \\
\hline 43 & 58 & 595.137 & $1.00 \mathrm{E}+00$ & $0.00 \mathrm{E}+00$ \\
\hline 43 & 59 & 584.424 & $1.00 \mathrm{E}+00$ & $0.00 \mathrm{E}+00$ \\
\hline 43 & 61 & 540.125 & $1.00 \mathrm{E}+00$ & $0.00 \mathrm{E}+00$ \\
\hline 44 & 46 & 10483.53 & $1.00 \mathrm{E}+00$ & $0.00 \mathrm{E}+00$ \\
\hline 44 & 47 & 8734.963 & $1.00 \mathrm{E}+00$ & $0.00 \mathrm{E}+00$ \\
\hline 44 & 50 & 1507.229 & $1.00 \mathrm{E}+00$ & $0.00 \mathrm{E}+00$ \\
\hline 44 & 51 & 1463.303 & $1.00 \mathrm{E}+00$ & $0.00 \mathrm{E}+00$ \\
\hline 44 & 54 & 1381.751 & $1.00 \mathrm{E}+00$ & $0.00 \mathrm{E}+00$ \\
\hline 44 & 55 & 1368.702 & $1.00 \mathrm{E}+00$ & $0.00 \mathrm{E}+00$ \\
\hline 44 & 56 & 1352.727 & $1.00 \mathrm{E}+00$ & $0.00 \mathrm{E}+00$ \\
\hline 44 & 62 & 416.224 & $1.00 \mathrm{E}+00$ & $0.00 \mathrm{E}+00$ \\
\hline 44 & 63 & 414.002 & $1.00 \mathrm{E}+00$ & $0.00 \mathrm{E}+00$ \\
\hline 44 & 64 & 413.924 & $1.00 \mathrm{E}+00$ & $0.00 \mathrm{E}+00$ \\
\hline 45 & 47 & 12979.67 & $1.00 \mathrm{E}+00$ & $0.00 \mathrm{E}+00$ \\
\hline 45 & 51 & 1548.115 & $1.00 \mathrm{E}+00$ & $0.00 \mathrm{E}+00$ \\
\hline 45 & 52 & 1526.718 & $1.00 \mathrm{E}+00$ & $0.00 \mathrm{E}+00$ \\
\hline 45 & 54 & 1457.131 & $1.00 \mathrm{E}+00$ & $0.00 \mathrm{E}+00$ \\
\hline 45 & 55 & 1442.626 & $1.00 \mathrm{E}+00$ & $0.00 \mathrm{E}+00$ \\
\hline 45 & 56 & 1424.89 & $1.00 \mathrm{E}+00$ & $0.00 \mathrm{E}+00$ \\
\hline 45 & 57 & 1401.617 & $1.00 \mathrm{E}+00$ & $0.00 \mathrm{E}+00$ \\
\hline 45 & 62 & 422.813 & $1.00 \mathrm{E}+00$ & $0.00 \mathrm{E}+00$ \\
\hline 45 & 63 & 420.52 & $1.00 \mathrm{E}+00$ & $0.00 \mathrm{E}+00$ \\
\hline 45 & 64 & 420.44 & $1.00 \mathrm{E}+00$ & $0.00 \mathrm{E}+00$ \\
\hline 45 & 65 & 420.04 & $1.00 \mathrm{E}+00$ & $0.00 \mathrm{E}+00$ \\
\hline
\end{tabular}




\section{Continued}

\begin{tabular}{|c|c|c|c|c|}
\hline 46 & 48 & 8019.169 & $1.00 \mathrm{E}+00$ & $0.00 \mathrm{E}+00$ \\
\hline 46 & 58 & 656.597 & $1.00 \mathrm{E}+00$ & $0.00 \mathrm{E}+00$ \\
\hline 46 & 59 & 643.581 & $1.00 \mathrm{E}+00$ & $0.00 \mathrm{E}+00$ \\
\hline 46 & 61 & 590.27 & $1.00 \mathrm{E}+00$ & $0.00 \mathrm{E}+00$ \\
\hline 47 & 48 & 9469.121 & $1.00 \mathrm{E}+00$ & $0.00 \mathrm{E}+00$ \\
\hline 47 & 58 & 664.934 & $1.21 \mathrm{E}-01$ & $3.63 E+08$ \\
\hline 47 & 59 & 651.588 & $1.15 \mathrm{E}-01$ & $3.63 \mathrm{E}+08$ \\
\hline 47 & 60 & 634.326 & $1.37 \mathrm{E}+00$ & $4.56 \mathrm{E}+09$ \\
\hline 47 & 61 & 596.998 & $1.69 \mathrm{E}-02$ & $6.32 \mathrm{E}+07$ \\
\hline 48 & 50 & 2255.4 & $7.23 \mathrm{E}-04$ & $3.16 \mathrm{E}+05$ \\
\hline 48 & 51 & 2158.44 & $2.67 \mathrm{E}-02$ & $7.64 \mathrm{E}+06$ \\
\hline 48 & 55 & 1958.75 & $1.44 \mathrm{E}-01$ & $5.01 \mathrm{E}+07$ \\
\hline 48 & 63 & 455.507 & $1.21 \mathrm{E}-02$ & $7.75 \mathrm{E}+07$ \\
\hline 49 & & 25238.4 & $4.34 \mathrm{E}-05$ & $4.54 \mathrm{E}+02$ \\
\hline 49 & 61 & 653.673 & $3.09 \mathrm{E}-01$ & $4.82 \mathrm{E}+09$ \\
\hline 50 & 58 & 1047.21 & $1.10 \mathrm{E}-06$ & $2.23 \mathrm{E}+03$ \\
\hline 50 & 59 & 1014.48 & $3.79 \mathrm{E}-05$ & $8.20 \mathrm{E}+04$ \\
\hline 50 & 61 & 888.052 & $1.43 \mathrm{E}-04$ & 4.03E+05 \\
\hline 51 & 58 & 1069.51 & $2.46 \mathrm{E}-06$ & $2.87 \mathrm{E}+03$ \\
\hline 51 & 59 & 1035.4 & $3.44 \mathrm{E}-04$ & $4.28 \mathrm{E}+05$ \\
\hline 51 & 60 & 992.484 & $7.78 \mathrm{E}-06$ & $1.05 \mathrm{E}+04$ \\
\hline 51 & 61 & 904.042 & $1.37 \mathrm{E}-03$ & $2.24 \mathrm{E}+06$ \\
\hline 52 & 60 & 1001.48 & $2.97 \mathrm{E}-04$ & $2.20 \mathrm{E}+05$ \\
\hline 54 & 58 & 1117.73 & $3.38 \mathrm{E}-05$ & $2.58 \mathrm{E}+04$ \\
\hline 54 & 59 & 1080.53 & $3.03 \mathrm{E}-04$ & $2.48 \mathrm{E}+05$ \\
\hline 54 & 60 & 1033.87 & $1.24 \mathrm{E}-04$ & $1.10 \mathrm{E}+05$ \\
\hline 55 & 58 & 1126.41 & $1.20 \mathrm{E}-04$ & $1.26 \mathrm{E}+05$ \\
\hline 55 & 59 & 1088.64 & $6.65 \mathrm{E}-07$ & $7.49 \mathrm{E}+02$ \\
\hline 55 & 60 & 1041.3 & $7.16 \mathrm{E}-06$ & $8.81 \mathrm{E}+03$ \\
\hline 55 & 61 & 944.368 & $3.05 E-03$ & $4.57 \mathrm{E}+06$ \\
\hline 56 & 58 & 1137.47 & $8.13 \mathrm{E}-05$ & $5.99 \mathrm{E}+04$ \\
\hline 56 & 59 & 1098.97 & $5.31 \mathrm{E}-05$ & $4.19 E+04$ \\
\hline 56 & 60 & 1050.74 & $1.10 \mathrm{E}-05$ & $9.49 \mathrm{E}+03$ \\
\hline 57 & 60 & 1063.76 & $2.92 \mathrm{E}-05$ & $1.91 \mathrm{E}+04$ \\
\hline 58 & 62 & 1275.25 & $4.37 \mathrm{E}-01$ & $2.56 \mathrm{E}+08$ \\
\hline 58 & 63 & 1254.62 & $2.69 \mathrm{E}-02$ & $2.28 \mathrm{E}+07$ \\
\hline 58 & 64 & 1253.91 & $2.64 \mathrm{E}-03$ & $1.60 \mathrm{E}+06$ \\
\hline 59 & 62 & 1327.39 & $1.34 \mathrm{E}-04$ & $7.25 E+04$ \\
\hline 59 & 63 & 1305.06 & $2.45 \mathrm{E}-02$ & $1.92 \mathrm{E}+07$ \\
\hline 59 & 64 & 1304.28 & $4.30 \mathrm{E}-01$ & $2.41 \mathrm{E}+08$ \\
\hline 60 & 62 & 1405.3 & $2.63 \mathrm{E}-02$ & $1.27 \mathrm{E}+07$ \\
\hline 60 & 63 & 1380.29 & $1.36 \mathrm{E}-03$ & $9.50 \mathrm{E}+05$ \\
\hline 60 & 64 & 1379.42 & $2.08 \mathrm{E}-02$ & $1.04 \mathrm{E}+07$ \\
\hline 60 & 65 & 1375.12 & $5.53 \mathrm{E}-01$ & $2.17 \mathrm{E}+08$ \\
\hline 61 & 63 & 1597.66 & $2.16 \mathrm{E}-01$ & $1.13 \mathrm{E}+08$ \\
\hline
\end{tabular}




\section{Conclusions}

This paper presents calculations of fine structure levels, oscillator strengths, and radiative decay rates for Nelike $\mathrm{Ni}$ and $\mathrm{Cu}$ ions. We show that there is a good agreement between our results which were obtained by using COWAN code and the other values from NIST.

The analysis that has been presented in this work shows that electron collisional pumping (ECP) is suitable for attaining population inversion and offering the potential for laser emission in the spectral region between 50 and $1000 \AA$ from the Ni XIX and $\mathrm{Cu}$ XX. This class of lasers can be achieved under the suitable conditions of pumping power as well as electron density. If the positive gains obtained previously for some transitions in the ions under studies (Ni XIX and $\mathrm{Cu} \mathrm{XX)} \mathrm{together} \mathrm{with} \mathrm{the} \mathrm{calculated} \mathrm{parameters,} \mathrm{it} \mathrm{could} \mathrm{be} \mathrm{achieved} \mathrm{experi-}$ mentally. A successful low-cost electron collisional pumping XUV and soft X-ray lasers can be developed for various applications. The results have suggested the following laser transitions in the Ni XIX and Cu XX plasma ion, as the most promising laser emission lines in the XUV and soft X-ray spectral regions.

\section{References}

[1] Matthews, D.L. et al. (1985) Demonstration of a Soft X-Ray Amplifier. Physical Review Letters, 54, 110-113. http://dx.doi.org/10.1103/PhysRevLett.54.110

[2] Nilsen J., (1992) Journal of Quantitative Spectroscopy and Radiative Transfer. Journal of Quantitative Spectroscopy and Radiative Transfer, 47, 171-177. http://dx.doi.org/10.1016/0022-4073(92)90026-Z

[3] Sukewer, S., et al. (1985) Amplification of Stimulated Soft X-Ray Emission in a Confined Plasma Column. Physical Review Letters, 55, 1753-1756. http://dx.doi.org/10.1103/PhysRevLett.55.1753

[4] Christiansen, J., et al. (1974) MEDUSA a One-Dimensional Laser Fusion Code. Computer Physics Communications, 7, 271-287. http://dx.doi.org/10.1016/0010-4655(74)90027-7

[5] Zimmermann, G.B., et al. (1975) Numerical Simulation of Laser Initiated Fusion. Comments on Plasma Physics and Controlled Fusion, 11, 51.

[6] King, R.E., et al. (2001) Saturated X-Ray Lasers at 196 and $73 \AA$ Pumped by a Picosecond Traveling-Wave Excitation. Physical Review A, 64, 053810. http://dx.doi.org/10.1103/PhysRevA.64.053810

[7] Rocca, J.J., et al. (1994) Demonstration of a Discharge Pumped Table-Top Soft-X-Ray Laser. Physical Review Letters, 73, 2192.

[8] Feldhaus, J, et al. (1999) The VUV FEL Project at DESY: Plans for Improving the Photon Beam Characteristics by Feedback and Seeding. Institute of Physics Conference Series, 159, 553-556.

[9] Lemoff, B.E., et al. (1995) Demonstration of a 10-Hz Femtosecond-Pulse-Driven XUV Laser at $41.8 \mathrm{~nm}$ in Xe IX. Physical Review Letters, 74, 1574-1577. http://dx.doi.org/10.1103/PhysRevLett.74.1574

[10] Cowan, R.D. (1981) The Theory of Atomic Structure and Spectra. University of California Press, Berkeley.

[11] Hartree, D.R. and Salpeter, E.E. (1957) Quantum Mechanics of One- and Two-electron Atoms. Springer-Verlage, Berlin, New York.

[12] Sobelman, I. (1979) Atomic Spectra and Radiative Transitions. Springer, Berlin. http://dx.doi.org/10.1007/978-3-662-05905-0

[13] NIST Atomic Spectra Data Base Line Data (Energy Ordered), 2006. 